\title{
INTERACTION OF TWO CHARGES IN A UNIFORM MAGNETIC FIELD: II. SPATIAL PROBLEM
}

\author{
D. PINHEIRO AND R. S. MACKAY
}

Dedicated to the memory of John Greene.

\begin{abstract}
The interaction of two charges moving in $\mathbb{R}^{3}$ in a magnetic field $\boldsymbol{B}$ can be formulated as a Hamiltonian system with six degrees of freedom. Assuming that the magnetic field is uniform and the interaction potential has rotation symmetry we reduce this system to one with three degrees of freedom. For special values of the conserved quantities, choices of parameters or restriction to the coplanar case, we obtain systems with two degrees of freedom. Specialising to the case of Coulomb interaction, these reductions enable us to obtain many qualitative features of the dynamics. For charges of the same sign, the gyrohelices either "bounce-back", "pass-through", or exceptionally converge to coplanar solutions. For charges of opposite signs, we decompose the state space into "free" and "trapped" parts with transitions only when the particles are coplanar. A scattering map is defined for those trajectories which come from and go to infinite separation along the field direction. It determines the asymptotic parallel velocities, guiding centre field lines, magnetic moments and gyrophases for large positive time from those for large negative time. In regimes where gyrophase averaging is appropriate, the scattering map has a simple form, conserving the magnetic moments and parallel kinetic energies (in a frame moving along the field with the centre of mass) and rotating or translating the guiding centre field lines. When the gyrofrequencies are in low order resonance, however, gyrophase averaging is not justified and transfer of perpendicular kinetic energy is shown to occur. In the extreme case of equal gyrofrequencies an additional integral helps us to analyse further and prove that there is typically also transfer between perpendicular and parallel kinetic energy.
\end{abstract}

\section{INTRODUCTION}

In this paper we analyse the interaction of two charged particles moving in three-dimensional space under the action of a uniform magnetic field and an interaction potential depending only on the distance between the particles. This problem is important for plasma physics and for atomic physics in magnetic fields. Apart from [4], where the separation of the centre of mass is treated in the quantum-mechanical setting, attention has tended to focus on some limiting regimes such as very strong magnetic field or plasmas with all the particles of the same kind (see $[2,7,8,20]$ ) or with one heavy particle idealized as fixed (the diamagnetic Kepler Problem, see $[9,11,24]$ ) or the case with charges summing to zero (see [21, 22]). The problem of Coulomb scattering of a charged particle by a fixed charge or two identical particles in a very strong magnetic field is treated in [23] in the quantum-mechanical setting. We will study the dynamics of two charged particles in a uniform magnetic field without making restrictions on the sizes of the magnetic field, the charges or the masses, except that we will assume that the particles behave classically and that their velocities and accelerations are small enough that we can neglect any relativistic and radiation effects. Although it is well known that non-uniformity of the magnetic field 
introduces further significant effects, we believe that there is value in establishing firm results for the uniform case first, which seems not yet to have been done in detail.

In [19] we made a detailed study of the problem of the interaction of two charged particles moving in a plane under the effect of a uniform perpendicular magnetic field. We assumed that the interaction between the particles was given by a potential depending on only the distance between the two particles. The problem can be formulated as a Hamiltonian system with four degrees of freedom. We made extensive use of the symmetries to obtain a reduction to two degrees of freedom. In the special case of same sign charges with equal gyrofrequencies (equal ratio of charge to mass) or on some special submanifolds we proved that this system is integrable. We then specialized our analysis to the most physically relevant case of a Coulomb-like potential. Analysing the reduced systems and the associated reconstruction maps we provided a detailed description for the regimes of parameters and level sets of the conserved quantities where bounded and unbounded motion are possible and we identified the cases where close approaches between the two particles are possible. Furthermore, we identified regimes where the system is non-integrable and contains chaos by proving the existence of invariant subsets containing a suspension of a non-trivial subshift.

The motion of one particle moving in three-dimensional space under the action of a uniform magnetic field is simple. It is the composition of two motions: a drift with constant velocity in the direction of the magnetic field and a uniform rotation in a plane orthogonal to the field about a fixed centre - the guiding centre, with constant radius - gyroradius, and angular velocity - gyrofrequency. Choosing the magnetic field to be vertical and oriented upwards, the motion in the circle is clockwise if the charge is positive and anticlockwise otherwise. We sign the gyrofrequency according to the direction of rotation. This problem can be formulated as a three degrees of freedom Hamiltonian system. It has symmetry under a four-dimensional subgroup of the Special Euclidean group of $\mathbb{R}^{3}$ (three-dimensional translations and a one-dimensional rotation). These symmetries induce conserved quantities for this system which is easily seen to be integrable.

One of the main goals of this paper is to study the scattering problem associated with the interaction of the two charges in the presence of a magnetic field and a Coulomb interaction potential:

$$
V(R)=\frac{e_{1} e_{2}}{4 \pi \epsilon_{0}} \frac{1}{R}
$$

where $R$ denotes the distance between the two particles, $e_{1}$ and $e_{2}$ denote the values of the charges and $\epsilon_{0}$ denotes the permittivity of the vacuum. If there is a large distance between the particles then the interaction is negligible and in this case the two particles move freely as described above. If the distance between the two particles is small then the strength of the interaction can not be neglected anymore and the particles interact. We will be looking at the situation where the particles have initially a large vertical separation and both move freely towards each other so that the particles start interacting when they get closer and then start moving apart until both particles move again like free particles. The goal is to describe the changes in their trajectories due to this interaction. For background on scattering in classical mechanics see the review [17] and references therein. 
We start section 2 by formulating our problem as a Hamiltonian system with a non-canonical symplectic form (see [13]), that makes it easier to see the system symmetries. We identify translational and rotational symmetries of the system and the corresponding conserved quantities. Furthermore, we prove the existence of an exceptional conserved quantity when the two particles have the same gyrofrequency.

We start section 3 by proving that the problem of the interaction of two particles in a magnetic field can be reduced to one with three degrees of freedom. Furthermore, when the two particles have the same gyrofrequency we use the exceptional conserved quantity to reduce the system to two degrees of freedom. We also prove that if the sum of the two charges is zero the dynamics in the zero set of the linear momenta are described by a two degrees of freedom Hamiltonian system. We achieve these results by constructing a set of coordinates in which the system exhibits a reduction to three degrees of freedom, and two degrees of freedom when it applies. This reduction is an extension to three-dimensional space of similar reductions obtained for the planar case in [19] (and for a similar problem in [10]). In the present paper the total change of coordinates that exhibits the reduction is computed. This change of coordinates is just the lift of a $S E(3)$ subgroup that, given the base dynamics of the reduced Hamiltonian systems, enables us to describe the full twelve-dimensional dynamics. The planar case is obtained as an invariant plane of the three-dimensional problem.

In section 4, we specialize our analysis of the problem to the Coulomb potential (1.1). We give a description of the reduced Hamiltonian systems obtained in section 3 with the generic potential $V$ replaced by the Coulomb potential, including:

- recalling from [19] the principal features of the dynamics on the invariant plane associated with the interaction of two particles moving in a plane under the action of a uniform perpendicular magnetic field.

- proving boundedness of some of the variables on the reduced space.

Using this information we obtain a classification of the various distinct types of dynamical behaviour in this system, distinguishing between three main cases: dynamics on the invariant plane, dynamics of two charges of the same sign and dynamics of two charges of opposite sign.

We start section 5 with an analysis of the scattering map associated with this problem in the limit where the two particles' trajectories are widely separated. We separate our analysis into two cases: rationally independent gyrofrequencies and rationally dependent gyrofrequencies. If the two particles' gyrofrequencies are rationally independent we obtain that the magnetic moments of the particles are adiabatic invariants and under the adiabatic approximation the vertical kinetic energy in the centre of mass frame is unchanged and the guiding centres have the following dynamics:

i) in the case of two charges whose sum is not zero, the guiding field lines rotate by some angle about a fixed field line during an interaction,

ii) in the case of two charges which sum to zero, the guiding field lines translate by some amount in a direction determined by the conserved quantities.

If the two particles' gyrofrequencies are rationally dependent, however (or one goes beyond the above adiabatic approximation), some transfer can occur between the horizontal kinetic energies 
of the two particles; there can also be a weaker exchange between horizontal and vertical kinetic energy. Indeed we prove both such transfers are typically non-zero when the gyrofrequencies are equal, and the first occurs when they sum to zero. If the gyrofrequencies differ in absolute value, we bound any such transfer by the fourth or higher inverse power of the distance between the gyrohelices. The results obtained in this limit agree with the more general qualitative description provided in section 4. Furthermore, we prove that in the case of "bouncing-back" behaviour, even if the vertical kinetic energy is conserved in the centre of mass frame, there is a transfer of vertical kinetic energy between the particles when the vertical centre of mass velocity is non-zero. We finish this section with a numerical study of the scattering map without using the assumption that the two particles' trajectories are widely separated. We observe regular behaviour for large energies and chaotic scattering for small positive energies.

\section{Problem Formulation}

We consider two particles with positive masses $m_{1}$ and $m_{2}$ and non-zero charges $e_{1}$ and $e_{2}$, respectively, moving in $\mathbb{R}^{3}$ under the action of a uniform magnetic field $\boldsymbol{B}=(0,0, B)$. Each of the particles is subject to a Lorentz force $\boldsymbol{F}_{\boldsymbol{L}}=e_{i} \boldsymbol{v}_{i} \times \boldsymbol{B}$ where $\boldsymbol{v}_{i}=\left(v_{x_{i}}, v_{y_{i}}, v_{z_{i}}\right) \in \mathbb{R}^{3}$ is the $i$-th particle velocity $(i \in\{1,2\})$ and $\times$ denotes the vector product between vectors of $\mathbb{R}^{3}$. Furthermore, we assume that the two particles' interaction is determined by a potential $V(r)$ depending on the distance $r$ between the two particles (invariance under translations and horizontal rotations would suffice).

The phase space $M$ for this problem is $\mathbb{R}^{12}$ with the singular points of the interaction potential removed (nine-dimensional planes if $V$ is the Coulomb potential (1.1)). Let $\boldsymbol{q}_{i}=\left(q_{x_{i}}, q_{y_{i}}, q_{z_{i}}\right) \in$ $\mathbb{R}^{3}$ denote the vector position of the $i$-th particle and $\boldsymbol{p}_{i}=\left(p_{x_{i}}, p_{y_{i}}, p_{z_{i}}\right) \in \mathbb{R}^{3}$ denote its (nonconjugate) momentum

$$
\boldsymbol{p}_{i}=m \boldsymbol{v}_{i}, \quad i \in\{1,2\} .
$$

The motion of the two particles can be described by a Hamiltonian system, with Hamiltonian function $H: M \longrightarrow \mathbb{R}$ and non-canonical symplectic form $\omega$ (see [13]), given by

$$
\begin{aligned}
H & =\frac{1}{2 m_{1}}\left|\boldsymbol{p}_{1}\right|^{2}+\frac{1}{2 m_{2}}\left|\boldsymbol{p}_{2}\right|^{2}+V\left(\left|\boldsymbol{q}_{1}-\boldsymbol{q}_{2}\right|\right) \\
\omega & =\sum_{i=1,2} \mathrm{~d} q_{x_{i}} \wedge \mathrm{d} p_{x_{i}}+\mathrm{d} q_{y_{i}} \wedge \mathrm{d} p_{y_{i}}+\mathrm{d} q_{z_{i}} \wedge \mathrm{d} p_{z_{i}}+k_{i} \mathrm{~d} q_{x_{i}} \wedge \mathrm{d} q_{y_{i}},
\end{aligned}
$$

where, for simplicity of notation, we introduce the constants

$$
k_{i}=-e_{i} B, \quad i \in\{1,2\}
$$

The Poisson bracket associated with this symplectic form, $\{.,\}:. C^{\infty}(M) \times C^{\infty}(M) \rightarrow C^{\infty}(M)$, is given by

$$
\begin{aligned}
\{F, G\}= & \sum_{i=1,2} \frac{\partial F}{\partial q_{x_{i}}} \frac{\partial G}{\partial p_{x_{i}}}-\frac{\partial G}{\partial q_{x_{i}}} \frac{\partial F}{\partial p_{x_{i}}}+\frac{\partial F}{\partial q_{y_{i}}} \frac{\partial G}{\partial p_{y_{i}}}-\frac{\partial G}{\partial q_{y_{i}}} \frac{\partial F}{\partial p_{y_{i}}}+\frac{\partial F}{\partial q_{z_{i}}} \frac{\partial G}{\partial p_{z_{i}}}-\frac{\partial G}{\partial q_{z_{i}}} \frac{\partial F}{\partial p_{z_{i}}} \\
& -k_{i}\left(\frac{\partial F}{\partial p_{x_{i}}} \frac{\partial G}{\partial p_{y_{i}}}-\frac{\partial G}{\partial p_{x_{i}}} \frac{\partial F}{\partial p_{y_{i}}}\right) .
\end{aligned}
$$


The Hamiltonian system defined by (2.1) is invariant under the following families of symmetries

$$
\begin{aligned}
& \phi_{\boldsymbol{v}}\left(\boldsymbol{q}_{1}, \boldsymbol{q}_{2}, \boldsymbol{p}_{1}, \boldsymbol{p}_{2}\right)=\left(\boldsymbol{q}_{1}+\boldsymbol{v}, \boldsymbol{q}_{2}+\boldsymbol{v}, \boldsymbol{p}_{1}, \boldsymbol{p}_{2}\right) \\
& \phi_{\theta}\left(\boldsymbol{q}_{1}, \boldsymbol{q}_{2}, \boldsymbol{p}_{1}, \boldsymbol{p}_{2}\right)=\left(R_{\theta} \boldsymbol{q}_{1}, R_{\theta} \boldsymbol{q}_{2}, R_{\theta} \boldsymbol{p}_{1}, R_{\theta} \boldsymbol{p}_{2}\right),
\end{aligned}
$$

where $\boldsymbol{v}=\left(v_{x}, v_{y}, v_{z}\right) \in \mathbb{R}^{3}$ is a translation vector and $R_{\theta}$ is the matrix representing a rotation in $\mathbb{R}^{3}$ of angle $\theta$ about the $z$ axis:

$$
R_{\theta}=\left(\begin{array}{ccc}
\cos \theta & -\sin \theta & 0 \\
\sin \theta & \cos \theta & 0 \\
0 & 0 & 1
\end{array}\right)
$$

We define the (signed) gyrofrequency $\Omega_{i}$ of each particle as

$$
\Omega_{i}=\frac{k_{i}}{m_{i}}, \quad i \in\{1,2\},
$$

and introduce the notation $\overline{\mathbf{J}}$ and $\mathbf{I}$ for the $3 \times 3$ matrices given by

$$
\overline{\mathbf{J}}=\left(\begin{array}{ccc}
0 & 1 & 0 \\
-1 & 0 & 0 \\
0 & 0 & 0
\end{array}\right), \quad \mathbf{I}=\left(\begin{array}{ccc}
1 & 0 & 0 \\
0 & 1 & 0 \\
0 & 0 & 0
\end{array}\right)
$$

Proposition 2.1. The Hamiltonian system (2.1) has the following conserved quantities:

- Linear momentum, given by

$$
\boldsymbol{P}_{3}=\left(P_{x}, P_{y}, P_{z}\right)=\boldsymbol{p}_{1}+\boldsymbol{p}_{2}+\overline{\mathbf{J}}\left(k_{1} \boldsymbol{q}_{1}+k_{2} \boldsymbol{q}_{2}\right) .
$$

- Angular momentum, given by

$$
L=\sum_{i=1,2} \boldsymbol{q}_{i} \cdot \overline{\mathbf{J}} \boldsymbol{p}_{i}-\frac{k_{i}}{2}\left|\mathbf{I} \boldsymbol{q}_{i}\right|^{2} .
$$

Furthermore, if the particles have equal gyrofrequencies $\Omega_{1}=\Omega_{2}$, there exists another conserved quantity $W$, given by

$$
W=\left|\mathbf{I}\left(\boldsymbol{p}_{1}+\boldsymbol{p}_{2}\right)\right|^{2} .
$$

The following commutation relations hold between the above conserved quantities:

$$
\begin{array}{lll}
\left\{P_{x}, P_{y}\right\}=k_{1}+k_{2}, & \left\{P_{x}, P_{z}\right\}=0, \quad\left\{P_{y}, P_{z}\right\}=0, \\
\left\{L, P_{x}\right\}=P_{y}, & \left\{L, P_{y}\right\}=-P_{x}, & \left\{L, P_{z}\right\}=0, \\
\left\{W, P_{x}\right\}=0, & \left\{W, P_{y}\right\}=0, \quad\left\{W, P_{z}\right\}=0, \quad\{W, L\}=0 .
\end{array}
$$

For a proof of a similar statement (with the two particles moving on a plane) see [19].

We will use the notation

$$
\boldsymbol{P}=\left(P_{x}, P_{y}\right) \in \mathbb{R}^{2}
$$

for the $(x, y)$-components of the linear momentum $\boldsymbol{P}_{3}$ given in proposition 2.1 and will use the notation $P_{z}$ when referring to its $z$-component.

Remarks i) It is possible to treat the problem in canonical coordinates, as in $[4,21,22]$ for the case of charges summing to zero, but the symmetries are less clear. 
ii) The conserved quantities $\boldsymbol{P}_{3}$ and $L$ are, respectively, the usual linear and angular momenta for the two body problem with extra terms representing the presence of the magnetic field and hence the effect of the Lorentz force on the particles.

iii) Combining $P_{x}$ and $P_{y}$ into the conserved quantity

$$
P=|\boldsymbol{P}|^{2}=P_{x}^{2}+P_{y}^{2}
$$

we obtain the following commutation relations

$$
\{P, L\}=0, \quad\left\{P, P_{z}\right\}=0, \quad\{P, W\}=0,
$$

which together with the commutation relations in proposition 2.1 show $L, P, P_{z}$ and $W$ to be in involution.

iv) Corresponding to $W$ there is a "hidden" symmetry in the case of equal gyrofrequencies $\Omega_{1}=\Omega_{2}$, given by

$$
\begin{aligned}
& \boldsymbol{q}_{1} \rightarrow \boldsymbol{q}_{1}+\frac{1}{k_{1}+k_{2}}\left[R_{2\left(k_{1}+k_{2}\right) \phi}-\mathbf{I d}_{3 \times 3}\right] \overline{\mathbf{J}}\left(\boldsymbol{p}_{1}+\boldsymbol{p}_{2}\right) \\
& \boldsymbol{q}_{2} \rightarrow \boldsymbol{q}_{2}+\frac{1}{k_{1}+k_{2}}\left[R_{2\left(k_{1}+k_{2}\right) \phi}-\mathbf{I d}_{3 \times 3}\right] \overline{\mathbf{J}}\left(\boldsymbol{p}_{1}+\boldsymbol{p}_{2}\right) \\
& \boldsymbol{p}_{1} \rightarrow \boldsymbol{p}_{1}+\frac{k_{1}}{k_{1}+k_{2}}\left[R_{2\left(k_{1}+k_{2}\right) \phi}-\mathbf{I d}_{3 \times 3}\right]\left(\boldsymbol{p}_{1}+\boldsymbol{p}_{2}\right) \\
& \boldsymbol{p}_{2} \rightarrow \boldsymbol{p}_{2}+\frac{k_{2}}{k_{1}+k_{2}}\left[R_{2\left(k_{1}+k_{2}\right) \phi}-\mathbf{I d}_{3 \times 3}\right]\left(\boldsymbol{p}_{1}+\boldsymbol{p}_{2}\right),
\end{aligned}
$$

where $\phi \in \mathbb{R}, \mathbf{I d}_{3 \times 3}$ is the identity matrix in $\mathbb{R}^{3}$ and $R_{2\left(k_{1}+k_{2}\right) \phi}$ is of the form (2.2) with $\theta$ replaced by $2\left(k_{1}+k_{2}\right) \phi$.

v) If the interaction potential in (2.1) is chosen to be the Coulomb potential (1.1) (as we will do in section 4) then the scaling transformation given by

$$
\overline{\boldsymbol{q}}_{i}=\lambda \boldsymbol{q}_{i}, \quad \bar{t}=\lambda^{3 / 2} t, \quad \bar{B}=\lambda^{-3 / 2} B,
$$

where $\lambda>0$, transforms the Hamiltonian function and symplectic form (2.1) to $\bar{H}=$ $\lambda^{-1} H$ and $\bar{\omega}=\lambda^{1 / 2} \omega$. We could then choose $\lambda$ so that $\bar{B}=1$ by a rescaling of the level sets of the Hamiltonian function in (2.1). Furthermore, choosing $e_{1}$ and $m_{1}$ to be units of charge and mass, respectively, we could further reduce the number of parameters of (2.1) by two. The Hamiltonian system (2.1) would then depend on only the charge $e_{2}$, mass $m_{2}$ and physical constant $\epsilon_{0}$.

vi) If the particles were also under the action of an electric field $\boldsymbol{E}$ perpendicular to the magnetic field $\boldsymbol{B}$ then the centre of mass of the system would drift with constant velocity given by

$$
\boldsymbol{u}=\frac{\boldsymbol{E} \times \boldsymbol{B}}{|\boldsymbol{B}|^{2}} .
$$

Changing to a frame moving with velocity $\boldsymbol{u}$ we reduce this problem to (2.1). This point seems not to be appreciated by the large number of authors of papers on atoms in crossed electric and magnetic fields, who assume the nucleus can be regarded as fixed and thereby complicate the problem. 


\section{REDUCTION}

In this section we provide local coordinates that exhibit the reduction of the Hamiltonian system (2.1) to three degrees of freedom and identify the regimes of parameters and invariant subsets of $\mathbb{R}^{12}$ where the system can be reduced to two degrees of freedom. This reduction is valid for all potentials $V$ which depend only on the distance between the particles (or more generally have horizontal rotation symmetry). For simplicity of notation we introduce the combinations

$$
M=m_{1}+m_{2}, \quad m=\frac{m_{1} m_{2}}{m_{1}+m_{2}} .
$$

We change coordinates from positions $\boldsymbol{q}_{i}$ and momenta $\boldsymbol{p}_{i}$ to guiding centres $\boldsymbol{R}_{i}=\left(R_{x_{i}}, R_{y_{i}}\right) \in$ $\mathbb{R}^{2}$, gyroradii vector $\boldsymbol{\rho}_{i}=\left(\rho_{x_{i}}, \rho_{y_{i}}\right) \in \mathbb{R}^{2}$, relative vertical position $q_{z} \in \mathbb{R}$ and a conjugate momentum $p_{z} \in \mathbb{R}$, vertical centre of mass $C_{z} \in \mathbb{R}$ and vertical linear momentum $P_{z} \in \mathbb{R}$, by making

$$
\begin{aligned}
\boldsymbol{\rho}_{i} & =\frac{1}{k_{i}} \mathbf{J}\left(p_{x_{i}}, p_{y_{i}}\right) & \boldsymbol{R}_{i} & =\left(q_{x_{i}}, q_{y_{i}}\right)-\boldsymbol{\rho}_{i} \\
q_{z} & =q_{z_{1}}-q_{z_{2}} & p_{z} & =\left(m_{2} p_{z_{1}}-m_{1} p_{z_{2}}\right) / M \\
C_{z} & =\left(m_{1} q_{z_{1}}+m_{2} q_{z_{2}}\right) / M & P_{z} & =p_{z_{1}}+p_{z_{2}}
\end{aligned}
$$

where $\mathbf{J}$ is the standard symplectic matrix in $\mathbb{R}^{2}$, given by

$$
\mathbf{J}=\left(\begin{array}{cc}
0 & 1 \\
-1 & 0
\end{array}\right)
$$

Then, the Hamiltonian system (2.1) transforms to

$$
\begin{aligned}
H & =\frac{k_{1} \Omega_{1}}{2}\left|\boldsymbol{\rho}_{1}\right|^{2}+\frac{k_{2} \Omega_{2}}{2}\left|\boldsymbol{\rho}_{2}\right|^{2}+V(R)+\frac{p_{z}^{2}}{2 m}+\frac{P_{z}^{2}}{2 M} \\
\omega & =\sum_{i=1,2} k_{i}\left(\mathrm{~d} R_{x_{i}} \wedge \mathrm{d} R_{y_{i}}-\mathrm{d} \rho_{x_{i}} \wedge \mathrm{d} \rho_{y_{i}}\right)+\mathrm{d} q_{z} \wedge \mathrm{d} p_{z}+\mathrm{d} C_{z} \wedge \mathrm{d} P_{z}
\end{aligned}
$$

where $R=\left(\left|\boldsymbol{R}_{1}-\boldsymbol{R}_{2}+\boldsymbol{\rho}_{1}-\boldsymbol{\rho}_{2}\right|^{2}+q_{z}^{2}\right)^{1 / 2}$. This coordinate change reduces (2.1) by one degree of freedom by conservation of $P_{z}$ and elimination of $C_{z}$. The quantities $\boldsymbol{P}, L$ and $W$ are now given by

$$
\boldsymbol{P}=\mathbf{J}\left(k_{1} \boldsymbol{R}_{1}+k_{2} \boldsymbol{R}_{2}\right), \quad L=\sum_{i=1,2} \frac{k_{i}}{2}\left(\left|\boldsymbol{\rho}_{i}\right|^{2}-\left|\boldsymbol{R}_{i}\right|^{2}\right), \quad W=\left|k_{1} \boldsymbol{\rho}_{1}+k_{2} \boldsymbol{\rho}_{2}\right|^{2} .
$$

We separate our analysis into two cases: $k_{1}+k_{2} \neq 0$ and $k_{1}+k_{2}=0$.

3.1. Case $k_{1}+k_{2} \neq 0$. We introduce the combinations

$$
\mu=k_{1}+k_{2}, \quad e=\frac{k_{1} k_{2}}{k_{1}+k_{2}},
$$

and note that since $k_{1}+k_{2} \neq 0$ then $\mu$ is non-zero and $e$ is well-defined. We introduce the planar relative position $\boldsymbol{q}=\left(q_{x}, q_{y}\right) \in \mathbb{R}^{2}$ and a conjugate momentum $\boldsymbol{p}=\left(p_{x}, p_{y}\right) \in \mathbb{R}^{2}$, by making the change of coordinates

$$
\begin{array}{rlrl}
\boldsymbol{q} & =\boldsymbol{R}_{1}-\boldsymbol{R}_{2}+\boldsymbol{\rho}_{1}-\boldsymbol{\rho}_{2} & \boldsymbol{p}=\frac{e}{2} \mathbf{J}\left(\boldsymbol{R}_{1}-\boldsymbol{R}_{2}-\boldsymbol{\rho}_{1}+\boldsymbol{\rho}_{2}\right) \\
\boldsymbol{P} & =\mathbf{J}\left(k_{1} \boldsymbol{R}_{1}+k_{2} \boldsymbol{R}_{2}\right) & \boldsymbol{f}=-\mathbf{J}\left(k_{1} \boldsymbol{\rho}_{1}+k_{2} \boldsymbol{\rho}_{2}\right),
\end{array}
$$


where $\boldsymbol{f}=\left(f_{x}, f_{y}\right) \in \mathbb{R}^{2}$ and $\boldsymbol{P}=\left(P_{x}, P_{y}\right) \in \mathbb{R}^{2}$. The inverse transformation is given by

$$
\begin{aligned}
\boldsymbol{R}_{1}=\frac{1}{\mu}\left[\frac{k_{2}}{2}\left(\boldsymbol{q}-\frac{2}{e} \mathbf{J} \boldsymbol{p}\right)-\mathbf{J} \boldsymbol{P}\right] & \boldsymbol{R}_{2}=\frac{1}{\mu}\left[-\frac{k_{1}}{2}\left(\boldsymbol{q}-\frac{2}{e} \mathbf{J} \boldsymbol{p}\right)-\mathbf{J} \boldsymbol{P}\right] \\
\boldsymbol{\rho}_{1}=\frac{1}{\mu}\left[\frac{k_{2}}{2}\left(\boldsymbol{q}+\frac{2}{e} \mathbf{J} \boldsymbol{p}\right)+\mathbf{J} \boldsymbol{f}\right] & \boldsymbol{\rho}_{2}=\frac{1}{\mu}\left[-\frac{k_{1}}{2}\left(\boldsymbol{q}+\frac{2}{e} \mathbf{J} \boldsymbol{p}\right)+\mathbf{J} \boldsymbol{f}\right] .
\end{aligned}
$$

Combining (3.3) and (3.2) we obtain

$$
\begin{aligned}
H & =\frac{1}{2 m}\left(|\boldsymbol{p}|^{2}+p_{z}{ }^{2}\right)+\frac{e^{2}}{8 m}|\boldsymbol{q}|^{2}+\frac{e}{2 m} \boldsymbol{q} . \mathbf{J} \boldsymbol{p}+V(R) \\
& +\epsilon(2 \boldsymbol{p}-e \mathbf{J} \boldsymbol{q}) \cdot \boldsymbol{f}+\frac{k_{1} \Omega_{1}+k_{2} \Omega_{2}}{2 \mu^{2}}|\boldsymbol{f}|^{2}+\frac{P_{z}^{2}}{2 M},
\end{aligned}
$$

and

$$
\omega=\mathrm{d} q_{x} \wedge \mathrm{d} p_{x}+\mathrm{d} q_{y} \wedge \mathrm{d} p_{y}+\mathrm{d} q_{z} \wedge \mathrm{d} p_{z}+\mathrm{d} C_{z} \wedge \mathrm{d} P_{z}+\frac{1}{\mu}\left(\mathrm{d} P_{x} \wedge \mathrm{d} P_{y}-\mathrm{d} f_{x} \wedge \mathrm{d} f_{y}\right),
$$

where $R=\left(|\boldsymbol{q}|^{2}+q_{z}^{2}\right)^{1 / 2}$ and

$$
\epsilon=\frac{\Omega_{1}-\Omega_{2}}{2 \mu}
$$

measures the displacement from the set of parameters satisfying $\Omega_{1}=\Omega_{2}$. This coordinate change reduces (2.1) by a further degree of freedom by conservation (and elimination) of $P_{x}$ and $P_{y}$. The quantities $L$ and $W$ are now given by

$$
L=\boldsymbol{q} . \mathbf{J} \boldsymbol{p}+\frac{1}{2 \mu}\left(|\boldsymbol{f}|^{2}-|\boldsymbol{P}|^{2}\right), \quad W=|\boldsymbol{f}|^{2} .
$$

Since $\boldsymbol{P}$ is conserved we remove the $-|\boldsymbol{P}|^{2} /(2 \mu)$ term from the angular momentum, corresponding to a change in the level set of the angular momentum, and define the following conserved quantity

$$
p_{\theta}=\boldsymbol{q} . \mathbf{J} \boldsymbol{p}+\frac{1}{2 \mu}|\boldsymbol{f}|^{2} .
$$

A final change of coordinates makes the system canonical and exhibits the reduction to three degrees of freedom. It is given by writing

$$
\begin{aligned}
& \boldsymbol{q}=r \boldsymbol{e}_{r} \quad \boldsymbol{p}=p_{r} \boldsymbol{e}_{r}+\frac{2 \mu p_{\theta}-p_{\phi}}{2 \mu r} \boldsymbol{e}_{\theta} \\
& \boldsymbol{f}=p_{\phi}^{1 / 2} \boldsymbol{e}_{2 \mu \phi+\theta} \\
& P_{x}=\mu \Pi_{x} \quad P_{y}=\Pi_{y}
\end{aligned}
$$

where $\theta$ is the direction of $\boldsymbol{q}$, i.e.

$$
\boldsymbol{e}_{\boldsymbol{r}}=\cos \theta \boldsymbol{e}_{x}+\sin \theta \boldsymbol{e}_{y}, \quad \boldsymbol{e}_{\theta}=-\sin \theta \boldsymbol{e}_{x}+\cos \theta \boldsymbol{e}_{y},
$$

with $\boldsymbol{e}_{x}=(1,0) \in \mathbb{R}^{2}$ and $\boldsymbol{e}_{y}=(0,1) \in \mathbb{R}^{2}$ and $\phi$ is an angle of period $\pi / \mu$ representing the direction of $\boldsymbol{f}$ relative to that of $\boldsymbol{q}$. The vector $\boldsymbol{e}_{2 \mu \phi+\theta}$ is defined in the same way as $\boldsymbol{e}_{\theta}$ with $\theta$ replaced by $2 \mu \phi+\theta$. The coordinate change given in (3.6) is singular at $p_{\phi}=0$ since $\phi$ is undefined in this case. There is another coordinate singularity at $r=0$ that corresponds to collisions when $q_{z}=0$.

We obtain the following result. 
Theorem 3.1. Let $k_{1}+k_{2} \neq 0$. Then the Hamiltonian system (2.1) reduces to one with three degrees of freedom in the variables $\left(r, p_{r}, \phi, p_{\phi}, q_{z}, p_{z}\right)$, given by

$$
\begin{aligned}
H & =H_{0}+\epsilon H_{1} \\
\omega & =\mathrm{d} r \wedge \mathrm{d} p_{r}+\mathrm{d} \phi \wedge \mathrm{d} p_{\phi}+\mathrm{d} q_{z} \wedge \mathrm{d} p_{z}+\mathrm{d} \theta \wedge \mathrm{d} p_{\theta}+\mathrm{d} \Pi_{x} \wedge \mathrm{d} \Pi_{y}+\mathrm{d} C_{z} \wedge \mathrm{d} P_{z},
\end{aligned}
$$

where $H_{0}=H_{0}\left(r, p_{r}, p_{\phi}, q_{z}, p_{z}, p_{\theta}, P_{z}\right)$ is given by

$$
H_{0}=\frac{1}{2 m}\left(p_{r}{ }^{2}+p_{z}{ }^{2}\right)+\frac{1}{2 m}\left(\frac{2 \mu p_{\theta}-p_{\phi}}{2 \mu r}\right)^{2}+\frac{e^{2}}{8 m} r^{2}+\frac{e}{2 m}\left(p_{\theta}+\frac{p_{\phi}}{2 \mu}\right)+\frac{1}{2 M} P_{z}^{2}+V(R)
$$

$H_{1}=H_{1}\left(r, p_{r}, p_{\theta}, \phi, p_{\phi}\right)$ is given by

$$
H_{1}=p_{\phi}^{1 / 2}\left(\left(e r+\frac{2 \mu p_{\theta}-p_{\phi}}{\mu r}\right) \cos (2 \mu \phi)-2 p_{r} \sin (2 \mu \phi)\right)+\frac{k_{1}-k_{2}}{2 \mu} p_{\phi}
$$

and

$\epsilon=\frac{\Omega_{1}-\Omega_{2}}{2 \mu}, \quad R=\left(r^{2}+q_{z}^{2}\right)^{1 / 2}, \quad p_{\theta}=L+\frac{1}{2 \mu} P, \quad p_{\phi}=W, \quad\left(\mu \Pi_{x}, \Pi_{y}\right)=\boldsymbol{P}$.

The reconstruction map is given by

$$
\begin{aligned}
\boldsymbol{R}_{1} & =-\frac{\mathbf{J} \boldsymbol{P}}{\mu}+\frac{k_{2}}{2 \mu}\left(\left(r-\frac{2 \mu p_{\theta}-p_{\phi}}{k_{1} k_{2} r}\right) \boldsymbol{e}_{r}+\frac{2}{e} p_{r} \boldsymbol{e}_{\theta}\right) \\
\boldsymbol{R}_{2} & =-\frac{\mathbf{J} \boldsymbol{P}}{\mu}-\frac{k_{1}}{2 \mu}\left(\left(r-\frac{2 \mu p_{\theta}-p_{\phi}}{k_{1} k_{2} r}\right) \boldsymbol{e}_{r}+\frac{2}{e} p_{r} \boldsymbol{e}_{\theta}\right) \\
\boldsymbol{\rho}_{1} & =\frac{p_{\phi}^{1 / 2}}{\mu} \mathbf{J} \boldsymbol{e}_{2 \mu \phi+\theta}+\frac{k_{2}}{2 \mu}\left(\left(r+\frac{2 \mu p_{\theta}-p_{\phi}}{k_{1} k_{2} r}\right) \boldsymbol{e}_{r}-\frac{2}{e} p_{r} \boldsymbol{e}_{\theta}\right) \\
\boldsymbol{\rho}_{2} & =\frac{p_{\phi}^{1 / 2}}{\mu} \mathbf{J} \boldsymbol{e}_{2 \mu \phi+\theta}-\frac{k_{1}}{2 \mu}\left(\left(r+\frac{2 \mu p_{\theta}-p_{\phi}}{k_{1} k_{2} r}\right) \boldsymbol{e}_{r}-\frac{2}{e} p_{r} \boldsymbol{e}_{\theta}\right) \\
q_{z_{1}} & =C_{z}+\frac{m_{2}}{M} q_{z}, \quad q_{z_{2}}=C_{z}-\frac{m_{1}}{M} q_{z} \\
p_{z_{1}} & =\frac{m_{1}}{M} P_{z}+p_{z}, \quad p_{z_{2}}=\frac{m_{2}}{M} P_{z}-p_{z} .
\end{aligned}
$$

If the gyrofrequencies of the two particles are equal, i.e. $\Omega_{1}=\Omega_{2}$, we have $\epsilon=0$. Applying theorem 3.1 we see that $\phi$ is ignorable and so we obtain the following result.

Corollary 3.2. If $\Omega_{1}=\Omega_{2}$ the Hamiltonian system (2.1) reduces to one with two degree of freedom in the variables $\left(r, p_{r}, q_{z}, p_{z}\right)$, given by

$$
\begin{aligned}
H & =H_{0}\left(r, p_{r}, p_{\phi}, q_{z}, p_{z}, p_{\theta}, P_{z}\right) \\
\omega & =\mathrm{d} r \wedge \mathrm{d} p_{r}+\mathrm{d} \phi \wedge \mathrm{d} p_{\phi}+\mathrm{d} q_{z} \wedge \mathrm{d} p_{z}+\mathrm{d} C_{z} \wedge \mathrm{d} P_{z}+\mathrm{d} \theta \wedge \mathrm{d} p_{\theta}+\mathrm{d} \Pi_{x} \wedge \mathrm{d} \Pi_{y},
\end{aligned}
$$

where $H_{0}$ is as given in theorem 3.1 .

3.2. Case $k_{1}+k_{2}=0$. We now treat the case where the charges sum to zero. For simplicity of notation let

$$
\kappa=k_{1}=-k_{2}
$$

We make the change of coordinates

$$
\begin{array}{rlrl}
\boldsymbol{q} & =\boldsymbol{R}_{1}-\boldsymbol{R}_{2}+\boldsymbol{\rho}_{1}-\boldsymbol{\rho}_{2} & \boldsymbol{p} & =-\frac{\kappa}{2} \mathbf{J}\left(\boldsymbol{\rho}_{1}+\boldsymbol{\rho}_{2}\right) \\
\boldsymbol{C} & =-\frac{1}{2} \mathbf{J}\left(\boldsymbol{R}_{1}+\boldsymbol{R}_{2}+\boldsymbol{\rho}_{1}+\boldsymbol{\rho}_{2}\right) & \boldsymbol{\Pi} & =\kappa\left(\boldsymbol{R}_{1}-\boldsymbol{R}_{2}\right)
\end{array}
$$


where $\boldsymbol{q}=\left(q_{x}, q_{y}\right) \in \mathbb{R}^{2}$ is the relative position of the two particles, $\boldsymbol{p}=\left(p_{x}, p_{y}\right) \in \mathbb{R}^{2}$ a conjugate momentum, $\boldsymbol{C}=\left(C_{x}, C_{y}\right) \in \mathbb{R}^{2}$ and $\boldsymbol{\Pi}=\left(\Pi_{x}, \Pi_{y}\right) \in \mathbb{R}^{2}$. Inverting (3.9) we obtain

$$
\begin{array}{rlrl}
\boldsymbol{R}_{1} & =\frac{1}{2 \kappa} \boldsymbol{\Pi}+\mathbf{J} \boldsymbol{C}-\frac{1}{\kappa} \mathbf{J} \boldsymbol{p} & \boldsymbol{R}_{2}=-\frac{1}{2 \kappa} \boldsymbol{\Pi}+\mathbf{J} \boldsymbol{C}-\frac{1}{\kappa} \mathbf{J} \boldsymbol{p} \\
\boldsymbol{\rho}_{1}=-\frac{1}{2 \kappa} \boldsymbol{\Pi}+\frac{1}{2}\left(\boldsymbol{q}+\frac{2}{\kappa} \mathbf{J} \boldsymbol{p}\right) & \boldsymbol{\rho}_{2}=\frac{1}{2 \kappa} \boldsymbol{\Pi}-\frac{1}{2}\left(\boldsymbol{q}-\frac{2}{\kappa} \mathbf{J} \boldsymbol{p}\right) .
\end{array}
$$

From (3.2) and (3.10), we obtain a system determined by the Hamiltonian function

$$
\begin{aligned}
H= & \frac{1}{2 m}|\boldsymbol{p}|^{2}+\frac{\kappa^{2}}{8 m}|\boldsymbol{q}|^{2}+\frac{\left(m_{2}-m_{1}\right) \kappa}{2 m_{1} m_{2}} \boldsymbol{q} \cdot \mathbf{J} \boldsymbol{p}+V(R) \\
& -\left(\frac{\kappa}{4 m} \boldsymbol{q}+\frac{m_{2}-m_{1}}{2 m_{1} m_{2}} \mathbf{J} \boldsymbol{p}\right) \cdot \boldsymbol{\Pi}+\frac{1}{8 m}|\boldsymbol{\Pi}|^{2}+\frac{1}{2 m} p_{z}{ }^{2}+\frac{1}{2 M} P_{z}{ }^{2},
\end{aligned}
$$

where $R=\left(|\boldsymbol{q}|^{2}+q_{z}^{2}\right)^{1 / 2}$, and symplectic form

$$
\omega=\mathrm{d} q_{x} \wedge \mathrm{d} p_{x}+\mathrm{d} q_{y} \wedge \mathrm{d} p_{y}+\mathrm{d} q_{z} \wedge \mathrm{d} p_{z}+\mathrm{d} C_{x} \wedge \mathrm{d} \Pi_{x}+\mathrm{d} C_{y} \wedge \mathrm{d} \Pi_{y}+\mathrm{d} C_{z} \wedge \mathrm{d} P_{z},
$$

with the conserved quantities

$$
\boldsymbol{P}=\mathbf{J} \Pi, \quad L=q . \mathbf{J} p+C . \mathrm{J} \Pi
$$

The Hamiltonian system (3.11) is already reduced to three degrees of freedom by conservation of $\boldsymbol{\Pi}$ and $P_{z}$ and elimination of $\boldsymbol{C}$ and $C_{z}$. Unless $\boldsymbol{\Pi}=\mathbf{0}$ (or equivalently $\boldsymbol{P}=\mathbf{0}$ ), it is not possible to use the angular momentum $L$ to reduce further (3.11) since $L$ depends on the cyclic variables $\boldsymbol{C}$ and hence it is not a function defined on the reduced space. We make a final change of coordinates, given by

$$
\boldsymbol{q}=r \boldsymbol{e}_{r}, \quad \boldsymbol{p}=p_{r} \boldsymbol{e}_{r}+\frac{p_{\theta}}{r} \boldsymbol{e}_{\theta},
$$

where $\boldsymbol{e}_{r}$ and $\boldsymbol{e}_{\theta}$ are as given in (3.7). We obtain the following result.

Theorem 3.3. Let $k_{1}+k_{2}=0$. Then the Hamiltonian system (2.1) reduces to one with three degrees of freedom in the variables $\left(r, p_{r}, \theta, p_{\theta}, q_{z}, p_{z}\right)$, given by

$$
\begin{aligned}
H & =H_{0}+H_{1} \\
\omega & =\mathrm{d} r \wedge \mathrm{d} p_{r}+\mathrm{d} \theta \wedge \mathrm{d} p_{\theta}+\mathrm{d} q_{z} \wedge \mathrm{d} p_{z}+\mathrm{d} C_{x} \wedge \mathrm{d} \Pi_{x}+\mathrm{d} C_{y} \wedge \mathrm{d} \Pi_{y}+\mathrm{d} C_{z} \wedge \mathrm{d} P_{z},
\end{aligned}
$$

where $H_{0}=H_{0}\left(r, p_{r}, p_{\theta}, q_{z}, p_{z}, P_{z}\right)$ is given by

$$
H_{0}=\frac{1}{2 m}\left(p_{r}{ }^{2}+p_{z}{ }^{2}\right)+\frac{1}{2 m}\left(\frac{p_{\theta}}{r}\right)^{2}+\frac{\kappa^{2}}{8 m} r^{2}+\frac{1}{2 M} P_{z}^{2}+\frac{\left(m_{2}-m_{1}\right) \kappa}{2 m_{1} m_{2}} p_{\theta}+V(R)
$$

$H_{1}=H_{1}\left(r, p_{r}, \theta, p_{\theta}, \Pi_{x}, \Pi_{y}\right)$ is given by

$$
H_{1}=-\left(\left(\frac{\kappa}{4 m} r+\frac{m_{2}-m_{1}}{2 m_{1} m_{2}} \frac{p_{\theta}}{r}\right) \boldsymbol{e}_{r}-\frac{m_{2}-m_{1}}{2 m_{1} m_{2}} p_{r} \boldsymbol{e}_{\theta}\right) \cdot \boldsymbol{\Pi}+\frac{1}{8 m}|\boldsymbol{\Pi}|^{2}
$$

and

$$
R=\left(r^{2}+q_{z}^{2}\right)^{1 / 2}, \quad p_{\theta}=L-\boldsymbol{C} . \mathbf{J} \boldsymbol{\Pi}, \quad \boldsymbol{\Pi}=-\mathbf{J} \boldsymbol{P} .
$$


The reconstruction map is given by

$$
\begin{aligned}
\boldsymbol{R}_{1} & =\frac{1}{2 \kappa} \boldsymbol{\Pi}+\mathbf{J} \boldsymbol{C}-\frac{1}{\kappa} \frac{p_{\theta}}{r} \boldsymbol{e}_{r}+\frac{1}{\kappa} p_{r} \boldsymbol{e}_{\theta} \\
\boldsymbol{R}_{2} & =-\frac{1}{2 \kappa} \boldsymbol{\Pi}+\mathbf{J} \boldsymbol{C}-\frac{1}{\kappa} \frac{p_{\theta}}{r} \boldsymbol{e}_{r}+\frac{1}{\kappa} p_{r} \boldsymbol{e}_{\theta} \\
\boldsymbol{\rho}_{1} & =-\frac{1}{2 \kappa} \boldsymbol{\Pi}+\frac{1}{2}\left(r+\frac{2}{\kappa} \frac{p_{\theta}}{r}\right) \boldsymbol{e}_{r}-\frac{1}{\kappa} p_{r} \boldsymbol{e}_{\theta} \\
\boldsymbol{\rho}_{2} & =\frac{1}{2 \kappa} \boldsymbol{\Pi}-\frac{1}{2}\left(r-\frac{2}{\kappa} \frac{p_{\theta}}{r}\right) \boldsymbol{e}_{r}-\frac{1}{\kappa} p_{r} \boldsymbol{e}_{\theta} \\
q_{z_{1}} & =C_{z}+\frac{m_{2}}{M} q_{z}, \quad q_{z_{2}}=C_{z}-\frac{m_{1}}{M} q_{z} \\
p_{z_{1}} & =\frac{m_{1}}{M} P_{z}+p_{z}, \quad p_{z_{2}}=\frac{m_{2}}{M} P_{z}-p_{z} .
\end{aligned}
$$

If $\boldsymbol{P}=\mathbf{0}$ then $\boldsymbol{\Pi}=\mathbf{0}$ and hence $H_{1}$, as given in the statement of theorem 3.3, is identically zero. From theorem 3.3, we obtain the following result.

Corollary 3.4. If $k_{1}+k_{2}=0$ and $\boldsymbol{P}=\mathbf{0}$ then the Hamiltonian system (2.1) reduces to one with two degrees of freedom in the variables $\left(r, p_{r}, q_{z}, p_{z}\right)$, given by

$$
\begin{aligned}
H & =H_{0}\left(r, p_{r}, q_{z}, p_{z}, p_{\theta}, P_{z}\right) \\
\omega & =\mathrm{d} r \wedge \mathrm{d} p_{r}+\mathrm{d} q_{z} \wedge \mathrm{d} p_{z}+\mathrm{d} \theta \wedge \mathrm{d} p_{\theta}+\mathrm{d} C_{x} \wedge \mathrm{d} \Pi_{x}+\mathrm{d} C_{y} \wedge \mathrm{d} \Pi_{y}+\mathrm{d} C_{z} \wedge \mathrm{d} \Pi_{z},
\end{aligned}
$$

where $H_{0}$ is as given in theorem 3.3 .

Remarks i) In $[21,22]$ a reduction similar to the above is provided for the case $k_{1}+$ $k_{2}=0$, but done with canonical coordinates. The resulting reduced system is the same as the one given here, up to the choice of the direction of the magnetic field. The pseudomomentum $\boldsymbol{K}$ of $[21,22]$ is represented here by the linear momentum vector $\boldsymbol{P}$. The remaining variables are the relative position and conjugate momentum. We provide one extra transformation to write the system in variables involving the distance between the particles and conjugate momentum.

ii) The reduction done in this section is mostly regular, making it a standard illustration of the theory of symplectic reduction (see $[1,3,6,16]$ ). There are, however, level sets of the conserved quantities where the reduction is singular: the level sets of the form $2\left(k_{1}+k_{2}\right) L+|\boldsymbol{P}|^{2}=0$ in the case $k_{1}+k_{2} \neq 0$ and $L=0$ in the case $k_{1}+k_{2}=0$ have conical singularities that must be removed for the reduced space to be a smooth manifold. In [16] symplectic reduction by the symmetry group $S E(2)$ is discussed in a general setting and in [18] a detailed study is done for the symplectic reduction of the Hamiltonian system representing the interaction of charges moving in a plane under the action of a uniform magnetic field by its symmetry group $S E(2)$. The symplectic reduction for the spatial version of that problem is completely analogous.

3.3. Invariant plane $q_{z}=0, p_{z}=0$. In this section we note the existence of an invariant plane for the dynamics of the reduced Hamiltonian systems given in theorems 3.1 and 3.3, and corresponding invariant plane of the Hamiltonian system (2.1).

Lemma 3.5. The reduced Hamiltonian systems (3.8) and (3.12) given, respectively, in theorems 3.1 and 3.3 have an invariant plane determined by the conditions $q_{z}=0$ and $p_{z}=0$. 
Proof. From (3.8) and (3.12) we obtain

$$
\dot{q}_{z}=\frac{1}{m} p_{z}, \quad \dot{p}_{z}=-q_{z} \frac{V^{\prime}(R)}{R} .
$$

In particular we obtain $\dot{q}_{z}=\dot{p}_{z}=0$ if $q_{z}=p_{z}=0$. It follows that $q_{z}$ and $p_{z}$ remain equal to zero if they both start at zero.

Invariance of the plane $q_{z}=0, p_{z}=0$ under the dynamics of (3.8) and (3.12) corresponds to invariance of the (ten-dimensional) plane

$$
\Lambda=\left\{\left(\boldsymbol{q}_{1}, \boldsymbol{q}_{2}, \boldsymbol{p}_{1}, \boldsymbol{p}_{2}\right) \in \mathbb{R}^{12}: q_{z_{1}}=q_{z_{2}}, m_{2} p_{z_{1}}=m_{1} p_{z_{2}}\right\}
$$

under the dynamics of (2.1). Furthermore, on the invariant plane $\Lambda$ the dynamics of the Hamiltonian system (2.1) reduce by a further degree of freedom compared to the reduced systems in theorems 3.1 and 3.3. In fact, by setting $q_{z}=0, p_{z}=0$ in the Hamiltonian systems (3.8) and (3.12) we obtain reduced dynamical systems for the problem of the interaction of two charges moving in a plane under the action of a magnetic field (with a shift in the energy level sets by $\left.P_{z}^{2} /(2 M)\right)$.

\section{Reconstructed Dynamics for a Coulomb potential}

In this section we use the reduced Hamiltonian systems and the corresponding reconstruction maps obtained in section 3 to provide a qualitative description of the possible types of dynamics in the full twelve-dimensional phase space in terms of the properties of the dynamics of the reduced systems. Throughout this section we consider the interaction potential to be Coulomb

$$
V(R)=\frac{G}{R},
$$

where $R$ is the distance between the particles, $G=e_{1} e_{2} /\left(4 \pi \epsilon_{0}\right)$ and $\epsilon_{0}$ is the permittivity of the vacuum.

From theorems 3.1 and 3.3 we obtain that the vertical $z$-component of the centre of mass of the two particles moves with constant velocity $\dot{C}_{z}=P_{z} / M$. Hence, by a translation we can assume that $\dot{C}_{z}$ and $P_{z}$ are 0 . Furthermore, without loss of generality we will assume that $C_{z}=0$. This corresponds to considering the system as moving with the centre of mass of the $z$-component. Until section 5.3, we will assume this is the case. To extend the results in the sections below to non-zero $P_{z}$ it is enough to add a drift $P_{z} t / M$ to the vertical positions $q_{z_{1}}$ and $q_{z_{2}}$ of the particles (by the reconstruction maps of theorems 3.1 and 3.3).

4.1. Dynamics on the invariant plane $\Lambda$. For the Coulomb interaction, extensive results on the planar case were obtained in [19]. We summarize here the implications. The next two results follow from theorems 3.1 and 3.3, lemma 3.5 and the results in [19]. We skip their proof.

Corollary 4.1. Let $k_{1}+k_{2} \neq 0$ and restrict attention to the dynamics of (2.1) on $\Lambda$. Then

- the Hamiltonian system (2.1) reduces to one with two degrees of freedom in the variables $\left(r, p_{r}, \phi, p_{\phi}\right)$, given by the restriction of (3.8) to $q_{z}=0, p_{z}=0$.

- if $\Omega_{1}=\Omega_{2}$ the Hamiltonian system (2.1) reduces to one with one degree of freedom in the variables $\left(r, p_{r}\right)$, given by the restriction of (3.8) to $q_{z}=0, p_{z}=0$ (and $\epsilon=0$ ). 
- if $e_{1}$ and $e_{2}$ have opposite signs and $\Omega_{1}+\Omega_{2} \neq 0$ the Hamiltonian system (2.1) contains a suspension of a non-trivial subshift of finite type on all level sets of sufficiently high energy.

Corollary 4.2. Let $k_{1}+k_{2}=0$ and restrict attention to the dynamics of (2.1) on $\Lambda$. Then

- the Hamiltonian system (2.1) reduces to one with two degrees of freedom in the variables $\left(r, p_{r}, \theta, p_{\theta}\right)$, given by the restriction of (3.12) to $q_{z}=0, p_{z}=0$.

- if $\boldsymbol{P}=\mathbf{0}$ the Hamiltonian system (2.1) reduces to one with one degree of freedom in the variables $\left(r, p_{r}\right)$, given by the restriction of (3.12) to $q_{z}=0, p_{z}=0$ (and $\boldsymbol{P}=\mathbf{0}$ ).

- if $\boldsymbol{P} \neq \mathbf{0}$ and $\Omega_{1}+\Omega_{2} \neq 0$ the Hamiltonian system (2.1) contains a suspension of a non-trivial subshift of finite type on all level sets of sufficiently high energy.

Remark The existence of chaotic orbits was proved in [19] for $G m^{1 / 2}<\delta_{0}$ for some $\delta_{0}>0$ depending on the parameters $e_{1}, e_{2}, m_{1}, m_{2}$ (with $e_{1} e_{2}<0$ and $e_{1} / m_{1} \neq-e_{2} / m_{2}$ ), the magnetic field $\boldsymbol{B}$, the energy $H>0$, and the angular momentum $L$ or squared linear momentum $|\boldsymbol{P}|^{2}$ (according as $e_{1}+e_{2} \neq 0$ or $e_{1}+e_{2}=0$ ) in a certain range depending on the preceding quantities. We should have pointed out there that by a 4 -dimensional group of scaling symmetries (dimensional analysis) the functional dependence of $\delta_{0}$ can be reduced so that the condition can be written as

$$
\frac{E^{3} B}{M^{1 / 2} H^{3 / 2} \epsilon_{0}}<\delta_{1}\left(e_{2} / e_{1}, m_{2} / m_{1}, A\right),
$$

where $E=\left|e_{1}-e_{2}\right|$ and $A=\left(e_{1}+e_{2}\right) B L / M H$ or $A=|\boldsymbol{P}|^{2} / 2 M H$ according as $e_{1}+e_{2} \neq 0$ or $e_{1}+e_{2}=0$. Equivalently,

$$
H^{3 / 2}>\frac{E^{3} B}{M^{1 / 2} \epsilon_{0} \delta_{1}} .
$$

Taking $\delta_{2}\left(e_{2} / e_{1}, m_{2} / m_{1}\right)=\max \delta_{1}\left(e_{2} / e_{1}, m_{2} / m_{1}, A\right)$ over $A$ yields chaos on each energy level with

$$
H^{3 / 2}>\frac{E^{3} B}{M^{1 / 2} \epsilon_{0} \delta_{2}},
$$

specifying quantitatively the minimum energy required in corollaries 4.1 and 4.2 .

As was observed in [19] the reduced dynamics exhibit the following types of dynamical behaviour:

- In the integrable regimes the energy levels are foliated by periodic orbits.

- Close to the integrable regimes most of the periodic orbits cease to exist and all but a small fraction of the orbits in the energy levels are quasiperiodic and hence the dynamics still look regular.

- For opposite signs of charge (except for the case $\Omega_{1}+\Omega_{2}=0$ ) there is chaotic dynamics on all level sets of sufficiently high energy, which implies non-integrability.

The full dynamics in $\Lambda$ correspond to a drift of the two particles with constant and equal velocities $P_{z} / M$ in the $z$-direction. The dynamics in the $(x, y)$ plane are as described in [19]:

1) If $k_{1}+k_{2} \neq 0$ the dynamics in the $(x, y)$ plane are mostly quasiperiodic with three rationally independent frequencies. The particles rotate with these three frequencies about a fixed centre determined by the linear momenta. 
2) If $k_{1}+k_{2}=0$ periodic and quasiperiodic base dynamics lift to possibly unbounded motion in the $(x, y)$ plane corresponding to a combination of a drift and quasiperiodic dynamics. The quasiperiodic dynamics have, generically, two rationally independent frequencies.

3) Chaotic dynamics lift to chaotic dynamics in the $(x, y)$ plane. The motion (in the $(x, y)$ plane) is always bounded if $k_{1}+k_{2} \neq 0$ and typically unbounded otherwise.

4.2. Bounds on the motion. In this section we study the boundedness or not of various of the variables for the reduced systems given in theorems 3.1 and 3.3. This will give us important insight into distinct types of dynamical behaviour of (2.1), which will be of significance for the description of the global dynamics of the reconstructed system given in section 4.3 and the associated scattering problem.

We first state an auxiliary result, providing a regime of parameters where the planar distance $r$ between the two particles is bounded away from zero. We skip the proof, since it follows from an analysis of the Hamiltonian functions given in theorems 3.1 and 3.3.

Lemma 4.3. Let $k_{1}+k_{2} \neq 0$. If $k_{1} k_{2}>0$, or $k_{1} k_{2}<0$ and the value of the conserved quantity $p_{\theta}$ is fixed so that $\mu p_{\theta}<0$, the planar distance $r$ between the two particles is bounded away from zero, i.e. there exists $d>0$ such that $r(t)>d$ for all $t \in \mathbb{R}$.

Similarly, let $k_{1}+k_{2}=0$. If $\boldsymbol{P}=\mathbf{0}$ and $p_{\theta} \neq 0$, the planar distance $r$ between the two particles is bounded away from zero, i.e. there exists $d>0$ such that $r(t)>d$ for all $t \in \mathbb{R}$.

In the next two lemmas we identify the sets of parameters and level sets of the conserved quantities for which the distance between the two particles remains bounded or not. In [19] we gave similar results for the case where the two particles move in a plane.

Lemma 4.4. Let $k_{1}+k_{2} \neq 0$ and consider the reduced Hamiltonian system given in theorem 3.1. Then

(i) for every level set of the Hamiltonian function the dynamics of $r$ and $p_{\phi}$ are bounded for all time.

(ii) if $e_{1}$ and $e_{2}$ have equal signs, there exists $E^{\prime} \in \mathbb{R}$ such that for every level set $E<E^{\prime}$ of the Hamiltonian function the projection of the level set $\{H=E\}$ onto the $q_{z}$ direction is unbounded but bounded away from $q_{z}=0$, and for $E>E^{\prime}$ the projection of the level set $\{H=E\}$ onto the $q_{z}$ direction is unbounded. Furthermore, for every level set of the Hamiltonian function the dynamics of $p_{r}$ and $p_{z}$ are bounded for all time.

(iii) if $e_{1}$ and $e_{2}$ have opposite signs and the value of the conserved quantity $p_{\theta}$ is fixed so that $2 \mu p_{\theta}<0$, there exists $E^{\prime \prime} \in \mathbb{R}$ such that for every level set $E<E^{\prime \prime}$ of the Hamiltonian function the dynamics of $q_{z}$ are bounded and for every level set $E>E^{\prime \prime}$ the projection of the level set $\{H=E\}$ onto the $q_{z}$ direction is unbounded. Furthermore, for every level set of the Hamiltonian function the dynamics of $p_{r}$ and $p_{z}$ are bounded for all time.

(iv) if $e_{1}$ and $e_{2}$ have opposite signs and the value of the conserved quantity $p_{\theta}$ is fixed so that $2 \mu p_{\theta} \geq 0$, there exists $E^{\prime \prime} \in \mathbb{R}$ such that for every level set $E<E^{\prime \prime}$ of the Hamiltonian function the dynamics of $q_{z}$ are bounded and for every level set $E>E^{\prime \prime}$ the projection of the level set $\{H=E\}$ onto the $q_{z}$ direction is unbounded.

Proof. Item (i) follows trivially from the fact that $H \rightarrow \infty$ as $r \rightarrow \infty$ (and similarly for $p_{\phi}$ ). 
For the proof of the remaining items let us define the function $\mathcal{H}$ by

$$
\mathcal{H}=H-G\left(r^{2}+q_{z}^{2}\right)^{-1 / 2},
$$

where $H$ is given in theorem 3.1 with $V$ replaced by the Coulomb potential (1.1) and $G=$ $e_{1} e_{2} /\left(4 \pi \epsilon_{0}\right)$. It is crucial to point out that the function $\mathcal{H}$ does not depend on $q_{z}$ and is always bounded below. For simplicity of notation we consider $H$ and $\mathcal{H}$ just as functions of the variables in the reduced phase spaces and do not make their dependence on the conserved quantities $P_{z}$ and $p_{\theta}$ explicit.

We now prove item (ii). For the case of same sign charges the Hamiltonian function $H$ is bounded below. Denote its lower bound by

$$
E^{-}=\inf H\left(r, p_{r}, \phi, p_{\phi}, q_{z}, p_{z}\right) .
$$

By lemma 4.3 we obtain that $r$ is bounded away from 0 and hence $G\left(r^{2}+q^{2}\right)^{-1 / 2}$ is bounded. On lines where $r$ is constant $G\left(r^{2}+q_{z}^{2}\right)^{-1 / 2}$ increases at non-zero rate from 0 at the limit $q_{z} \rightarrow-\infty$ to attain its (positive) maximum at $q_{z}=0$ to decrease again to 0 at non-zero rate at the limit $q_{z} \rightarrow+\infty$. We now define

$$
E^{\prime}=\inf H\left(r, p_{r}, \phi, p_{\phi}, q_{z}=0, p_{z}\right),
$$

and note that $E^{-}<E^{\prime}$. From a simple analysis of the Hamiltonian function $H$ we obtain that for every level set $E^{-} \leq E<E^{\prime}$ of the Hamiltonian function the projection of the level set $\{H=E\}$ onto the $q_{z}$ direction is bounded away from 0 and for $E>E^{\prime}, q_{z}=0$ intersects the level sets of the Hamiltonian function. Furthermore, we obtain that for every level set of the Hamiltonian function the projection of the level set $\{H=E\}$ onto the $q_{z}$ direction is unbounded. Boundedness of the dynamics of $p_{r}$ and $p_{z}$ follow from the fact that $r$ is always bounded away from 0 (lemma 4.3).

For the proof of item (iii) we start by defining

$$
E^{\prime \prime}=\inf \mathcal{H}\left(r, p_{r}, \phi, p_{\phi}, p_{z}\right) .
$$

By lemma 4.3 we get that $r$ is bounded away from 0 and hence $G\left(r^{2}+q_{z}^{2}\right)^{-1 / 2}$ is bounded. On lines where $r$ is constant $G\left(r^{2}+q_{z}^{2}\right)^{-1 / 2}$ decreases at non-zero rate from 0 at the limit $q_{z} \rightarrow-\infty$ to its (negative) minimum at $q_{z}=0$ to increase again to 0 at non-zero rate at the limit $q_{z} \rightarrow+\infty$. We note that in this case the function $H$ is also bounded below and define

$$
\begin{aligned}
E^{-} & =\inf H\left(r, p_{r}, \phi, p_{\phi}, q_{z}=0, p_{z}\right) \\
& =\inf \left(\mathcal{H}\left(r, p_{r}, \phi, p_{\phi}, p_{z}\right)+\frac{G}{r}\right),
\end{aligned}
$$

and note that by item (i) in this lemma, $r$ is bounded and hence $G / r$ is strictly negative implying that $E^{-}<E^{\prime \prime}$. Using the fact that $\mathcal{H}$ does not depend on $q_{z}$ a simple analysis of the Hamiltonian function $H$ shows that for every level set $E^{-} \leq E<E^{\prime \prime}$ of $H$ the dynamics of $q_{z}$ are bounded for all time and for every level set $E>E^{\prime \prime}$ the projection of the level set $\{H=E\}$ onto the $q_{z}$ direction is unbounded. Boundedness of the dynamics of $p_{r}$ and $p_{z}$ follow from the fact that $r$ is always bounded away from 0 (lemma 4.3).

To prove (iv) we note that $H$ is not bounded below at points satisfying $p_{\phi}=2 \mu p_{\theta}$ and define

$$
E^{\prime \prime}=\inf \mathcal{H}\left(r, p_{r}, \phi, p_{\phi}, p_{z}\right) .
$$


Since $G\left(r^{2}+q_{z}^{2}\right)^{-1 / 2}<0$ (possibly unbounded) we obtain that $H \leq \mathcal{H}$. A simple analysis of the Hamiltonian function $H$ shows that for every level set $E<E^{\prime \prime}$ of $H$ the dynamics of $q_{z}$ are bounded for all time and for every level set $E>E^{\prime \prime}$ the projection of the level set $\{H=E\}$ onto the $q_{z}$ direction is unbounded.

We skip the proof of the next lemma which is analogous to (though simpler than) the proof of the previous lemma 4.4 .

Lemma 4.5. Let $k_{1}+k_{2}=0$ and consider the reduced Hamiltonian system given in theorem 3.3. Then

(i) for every level set of the Hamiltonian function the dynamics of $r$ and $p_{\theta}$ are bounded for all time.

(ii) if $\boldsymbol{P}=0$ and $p_{\theta} \neq 0$, there exists $E^{\prime \prime} \in \mathbb{R}$ such that for every level set $E<E^{\prime \prime}$ of the Hamiltonian function the dynamics of $q_{z}$ are bounded and for every level set $E>E^{\prime \prime}$ the projection of the level set $\{H=E\}$ onto the $q_{z}$ direction is unbounded. Furthermore, for every level set of the Hamiltonian function the dynamics of $p_{r}$ and $p_{z}$ are bounded for all time.

(iii) if $\boldsymbol{P} \neq 0$ or $\boldsymbol{P}=0$ and $p_{\theta}=0$, there exists $E^{\prime \prime} \in \mathbb{R}$ such that for every level set $E<E^{\prime \prime}$ of the Hamiltonian function the dynamics of $q_{z}$ are bounded and for every level set $E>E^{\prime \prime}$ the projection of the level set $\{H=E\}$ onto the $q_{z}$ direction is unbounded.

4.3. Description of the global dynamics of the reconstructed system. The dynamics of $\left(q_{z}, p_{z}\right)$ is given by

$$
\dot{q}_{z}=\frac{p_{z}}{m}, \quad \dot{p}_{z}=-q_{z} \frac{V^{\prime}(R)}{R} .
$$

Although these depend on $r(t)$ via $R=\sqrt{q_{z}^{2}+r^{2}}$, quite a lot can be deduced without explicit knowledge of $r(t)$. We separate the analysis according as the charges have the same or opposite signs.

4.3.1. Same sign charges. In this case $V^{\prime}<0$, so the signs of $\dot{q}_{z}$ and $\dot{p}_{z}$ in the $\left(q_{z}, p_{z}\right)$ plane are as indicated in Fig. 1, and the normal motion to $\Lambda$ is hyperbolic, as shown.

In particular, every trajectory in the quadrant $q_{z} \geq 0, p_{z} \leq 0$, except those on $\Lambda\left(q_{z}=p_{z}=0\right)$, has $q_{z}(t)$ decreasing, $p_{z}(t)$ increasing. Thus it has to do precisely one of the following:

(1) $\operatorname{cross} p_{z}=0, q_{z}>0$;

(2) $\operatorname{cross} q_{z}=0, p_{z}<0$;

(3) converge to $\Lambda$.

In the first case, $q_{z}(t)$ subsequently increases to $+\infty$, so we say the gyrating particles "bounceback". In the second case, $q_{z}(t)$ decreases to $-\infty$, so we say the gyrating particles "passthrough". The third case can be called the stable set of $\Lambda$. Only a set of measure zero on each energy level does the third case, because $\Lambda_{E}$, the intersection of $\Lambda$ with energy level $E$, has measure zero in the energy level, and volume is conserved.

We conjecture that $\Lambda$ (and thus $\Lambda_{E}$ for all regular values of $E$ ) is normally hyperbolic, though we have proved this in only the special case of equal gyrofrequencies (section (5.2.3)) (the difficulty in the general case is that unless measured in a suitable way, tangential contraction 


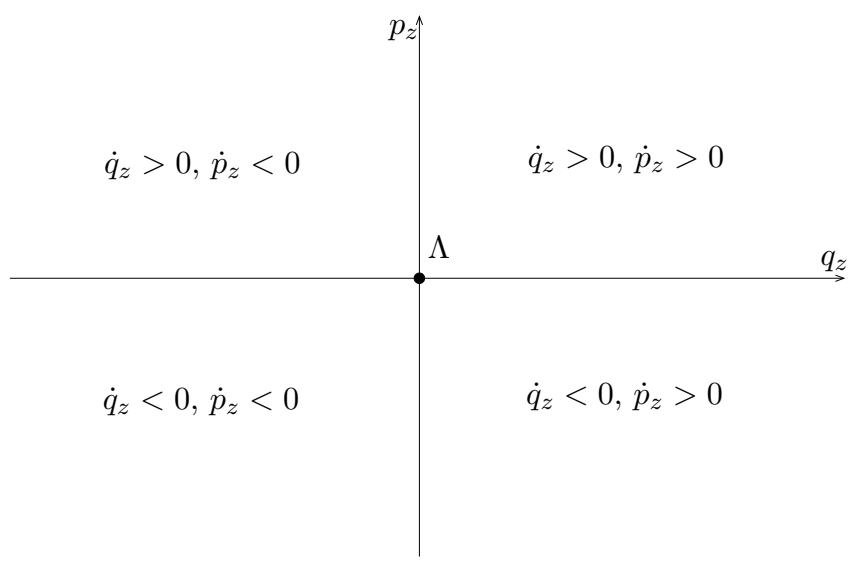

Figure 1: Signs of $\dot{q}_{z}$ and $\dot{p}_{z}$ for the case of same sign charges.

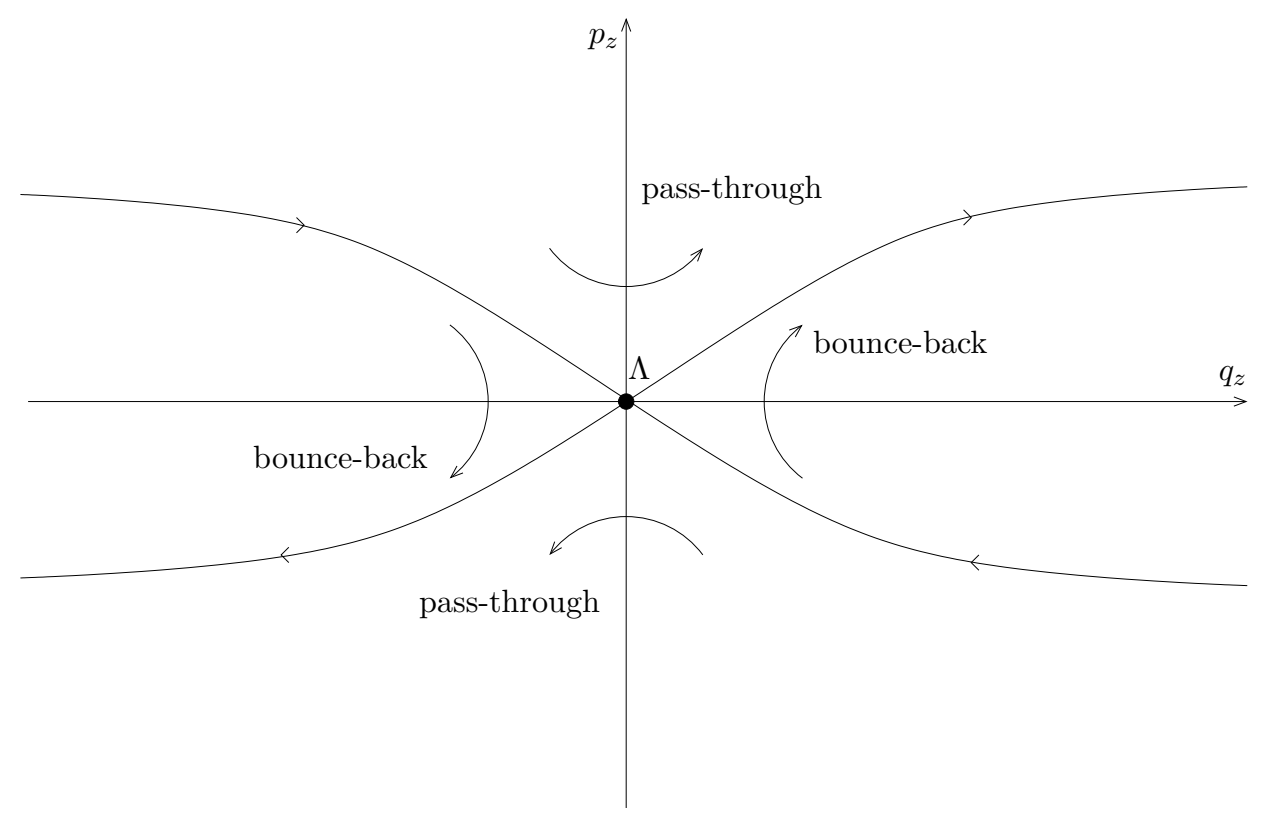

Figure 2: Sketch of the dynamics of $\left(q_{z}, p_{z}\right)$ for charges of the same sign. $\Lambda$ is the invariant set of coplanar states. The invariant manifolds of $\Lambda$ are 4D in each 5D energy level above the minimum energy on $\Lambda$ for the reduced system. They do not project to curves in $\left(q_{z}, p_{z}\right)$ but the directions of motion of $q_{z}, p_{z}$ are correctly represented. They separate trajectories from $q_{z}= \pm \infty$ into "bounce-back" and "pass-through".

might be almost as strong as normal contraction). If $\Lambda_{E}$ is normally hyperbolic then its stable set is a smooth submanifold of codimension one, and each trajectory on it converges to a trajectory on $\Lambda_{E}$. The stable manifold separates the cases of "bounce-back" and "pass-through". For energies in the interval $\left(E^{\prime}, E_{1}\right)$ up to the next critical value $E_{1}$ on $\Lambda, \Lambda_{E}$ is topologically a 3sphere and can be spanned by two $4 \mathrm{D}$ hemispheres of unidirectional flux in the $5 \mathrm{D}$ energy level (it suffices to take one hemisphere to be $q_{z}=0, p_{z}>0$, the other $q_{z}=0, p_{z}<0$ ), representing "pass-through" transitions in the two directions. The flux of energy-level-volume across the 
hemispheres is given by an action integral over $\Lambda_{E}$ [15]. For $E>E_{1}$ the topological type of $\Lambda_{E}$ changes, but the division into "pass-through" and "bounce-back" orbits persists.

4.3.2. Opposite sign charges. In this case $V^{\prime}>0$ so the signs of $\dot{q}_{z}$ and $\dot{p}_{z}$ are as in Fig. 3 and the normal motion to $\Lambda$ is elliptic.

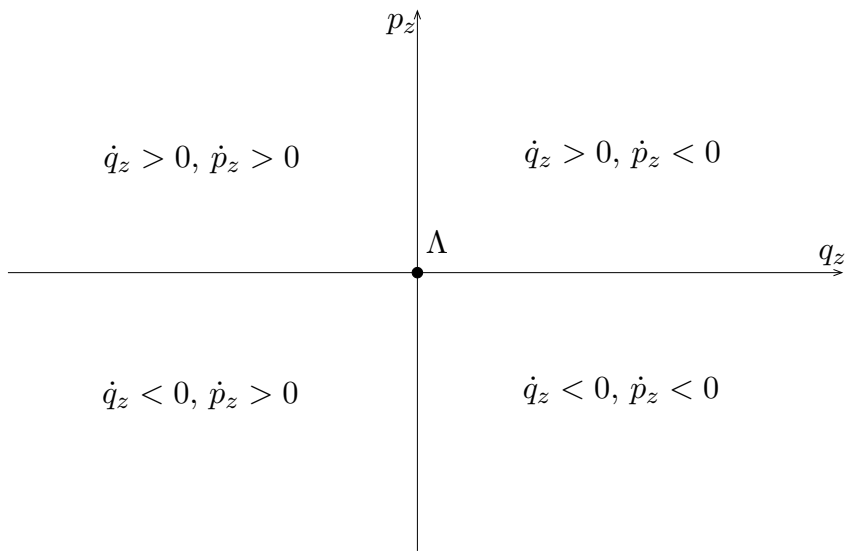

Figure 3: Signs of $\dot{q}_{z}$ and $\dot{p}_{z}$ for the case of opposite sign charges.

The key to understanding this case is to regularise the sets $\Omega_{ \pm}=\left\{q_{z}= \pm \infty, p_{z}=0\right\}$, by setting $q_{z}= \pm \sigma^{-2}$ (or using the global coordinate $\eta$ of Fig. 4) and using a new time $s$ with $d s / d t=\sigma^{3}$. Specialising to $\Omega_{+}$this yields

$$
\frac{\mathrm{d} \sigma}{\mathrm{d} s}=-\frac{p}{2 m}, \quad \frac{\mathrm{d} p}{\mathrm{~d} s}=-\frac{G \sigma}{\left(1+r^{2} \sigma^{4}\right)^{3 / 2}} .
$$

In particular, $\Omega_{+}$gives an invariant submanifold at $\sigma=p=0$, and the normal motion is hyperbolic. The dynamics on $\Omega_{+}$(and anywhere on $\sigma=0$ ) is not really defined since it has infinite gyrofrequencies in the new time $s$, but the guiding centre positions and the gyroradii are constant, so $\Omega_{+}$can be regarded as a normally hyperbolic submanifold. In particular, we conjecture that the stable and unstable sets for $\Omega_{+}$are smooth submanifolds of codimension one (a modification of usual proofs should work).

The stable and unstable manifolds of $\Omega_{ \pm}$, extended to $q_{z}=0$, separate the state space into a "trapped" region which reaches $q_{z}= \pm \infty$ at only $\Omega_{ \pm}$and two "free" regions which extend to $q_{z}= \pm \infty$ with $p_{z}>0$ and $p_{z}<0$ respectively. Although we have not proved it, we expect that the stable and unstable manifolds intersect at $q_{z}=0$ but do not coincide. Then the intersections of the invariant manifolds with $q_{z}=0$ form 3D lobes in each energy level, consisting of the flux between free and trapped motions. The fluxes are given by action integrals over the intersection submanifolds. Any trajectory other than those on an invariant manifold of $\Omega_{ \pm}$describes a sequence of transitions in the graph below.

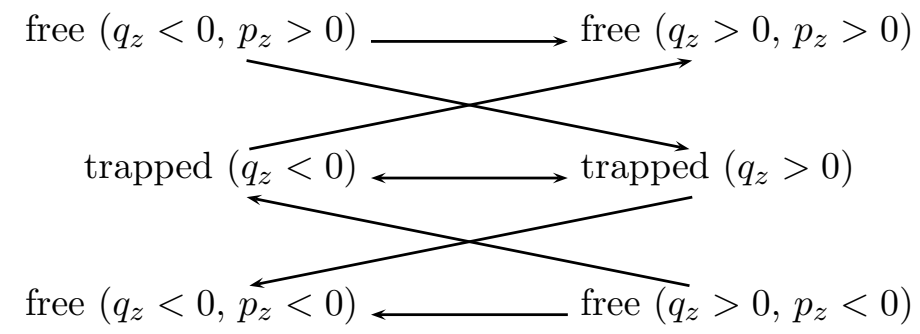


In particular there are:

(1) permanently trapped trajectories, e.g. the set $\Lambda\left(p_{z}=q_{z}=0\right)$ and all those with $E<E^{\prime \prime}$; we call these "atom-like" (thinking of the case of a proton and an electron); they can be subdivided into bounded trajectories and those with unbounded oscillation (oscillating between a neighbourhood of $\Lambda$ and unbounded excursions near $q_{z}= \pm \infty$ ); it would be interesting to prove existence of the latter;

(2) trajectories starting and ending free but possibly spending a finite time trapped in between; these are the main object of study for scattering; they may end in the same or opposite direction as they begin; the two cases can be called "pass-through" and "bounce-back".

(3) trajectories starting free and making a transition to permanently trapped; there could even be positive flux for this on each energy level with $E>E^{\prime \prime}$, because the volume of trapped orbits with given energy greater than $E^{\prime \prime}$ is infinite (owing to divergence of the integral as $q_{z} \rightarrow \infty$ ); these almost certainly lead to chaotic scattering in the neighbourhood, because they are likely to form a fractal and thus create a fractal structure of behaviour for the scattering trajectories.

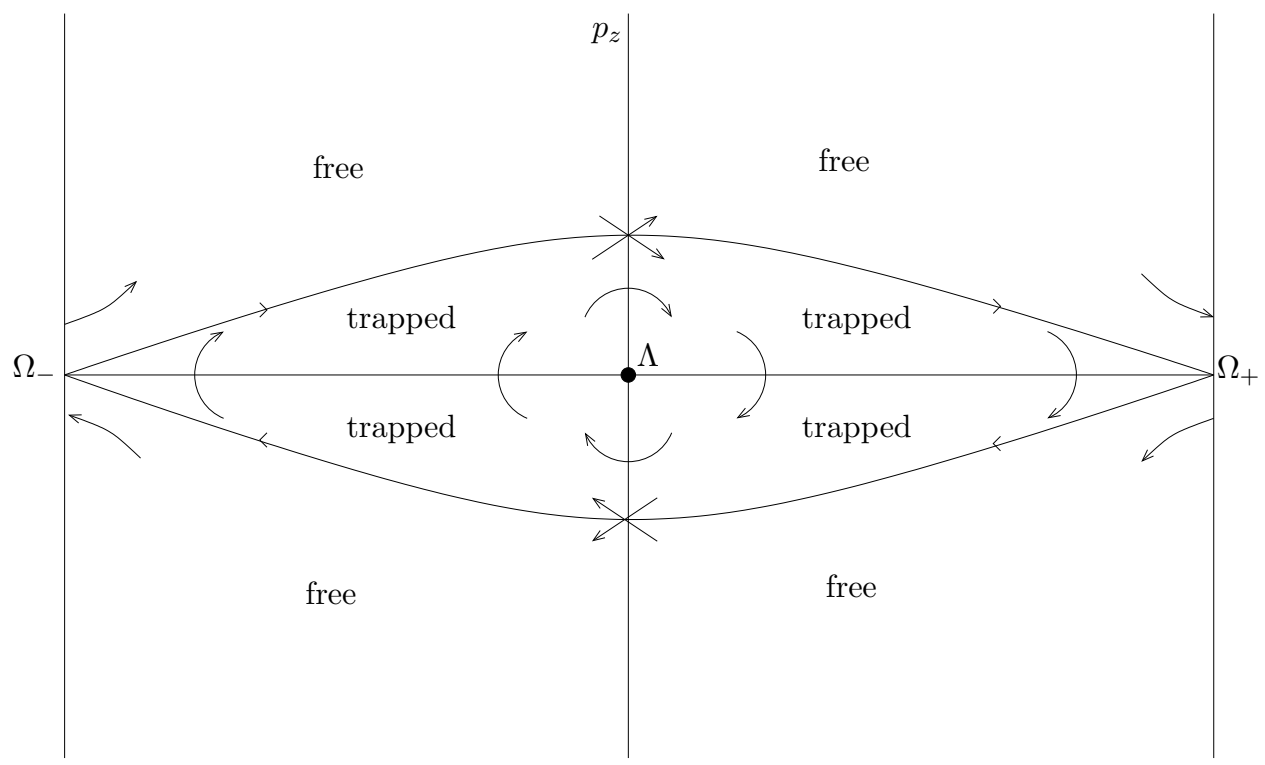

Figure 4: Sketch of the dynamics of $\left(q_{z}, p_{z}\right)$ for charges of opposite sign. The horizontal coordinate $\eta$ is related to $q_{z}$ by $q_{z}=\frac{\eta}{\left(1-\eta^{2}\right)^{2}}$. $\Omega_{ \pm}$are normally hyperbolic invariant sets at $q_{z}= \pm \infty$, $p_{z}=0$, for a regularised system with new time $s$ related by $\mathrm{d} s / \mathrm{d} t=\left(1-\eta^{2}\right)^{-3}$. The invariant manifolds for $\Omega_{ \pm}$are $4 \mathrm{D}$ in each $5 \mathrm{D}$ energy level above the minimum energy on $\Omega_{ \pm}$for the reduced system. They do not project to curves in $\left(q_{z}, p_{z}\right)$ but the directions of motion of $q_{z}, p_{z}$ are correctly represented. They separate each quadrant of $\left(q_{z}, p_{z}\right)$ into free and trapped subsets. They intersect the $4 \mathrm{D}$ plane $q_{z}=0$ in 3 -spheres. The two 3 -spheres with $p_{z}>0$ are expected to intersect but not coincide, so there is flux from free $q_{z}<0$ to trapped $q_{z}>0$ and from trapped $q_{z}<0$ to free $q_{z}>0$. Similarly for $p_{z}<0$. 
4.4. Summary of the different types of dynamics. In this section we combine the results given in the three previous subsections with the reconstruction maps of theorems 3.1 and 3.3 to provide a characterization of the dynamics of the Hamiltonian system (2.1).

As seen before, we have to distinguish between three main cases: dynamics on the invariant plane $\Lambda$, dynamics of two charges of the same sign and dynamics of two charges of opposite sign.

(1) Dynamics on the invariant plane $\Lambda$. In the invariant plane $\Lambda$ the two particles drift with equal and constant velocity in the vertical $z$-direction while the dynamics in the $(x, y)$ plane correspond to the interaction of the two particles in a plane. We call this "planar" behaviour.

(2) Dynamics of two charges of the same sign. The following types of dynamical behaviour have been identified:

(2.1) "bounce-back" behaviour - the dynamics are such that the following asymptotic conditions are satisfied:

a) $\lim _{t \rightarrow \pm \infty} q_{z_{1}}(t)=-\lim _{t \rightarrow \pm \infty} q_{z_{2}}(t)$.

b) $\lim _{t \rightarrow \pm \infty}\left|q_{z_{1}}(t)\right|=\lim _{t \rightarrow \pm \infty}\left|q_{z_{2}}(t)\right|=\infty$.

c) $q_{z_{1}}(t)-q_{z_{2}}(t)$ is bounded away from zero for all $t \in \mathbb{R}$.

(2.2) "pass-through" behaviour - the dynamics are such that the following asymptotic conditions are satisfied:

a) $\lim _{t \rightarrow \pm \infty} q_{z_{1}}(t)=-\lim _{t \rightarrow \pm \infty} q_{z_{2}}(t)$.

b) $\lim _{t \rightarrow \pm \infty}\left|q_{z_{1}}(t)\right|=\lim _{t \rightarrow \pm \infty}\left|q_{z_{2}}(t)\right|=\infty$.

c) $\lim _{t \rightarrow+\infty} q_{z_{i}}(t)=-\lim _{t \rightarrow-\infty} q_{z_{i}}(t), \quad i \in\{1,2\}$.

(2.3) trajectories forward or backward asymptotic to $\Lambda$.

(3) Dynamics of two charges of the opposite sign. The following types of dynamical behaviour have been identified

(3.1) "atom-like" behaviour - the particles are permanently trapped and can have either bounded or unbounded oscillations. However, even in the case of bounded oscillations, each particle position may be unbounded:

- if $P_{z} \neq 0$ the particles drift in the vertical direction with non-zero velocity.

- if $k_{1}+k_{2} \neq 0$ and $P_{z}=0$ using (3.1) and the reconstruction map in theorem 3.1 , we obtain

$$
\left(q_{x_{i}}, q_{y_{i}}\right)=-\frac{\mathbf{J} \boldsymbol{P}}{\mu}+(-1)^{i+1} \frac{1}{\mu}\left(k_{2} r \boldsymbol{e}_{r}+(-1)^{i+1} p_{\phi}^{1 / 2} \mathbf{J} \boldsymbol{e}_{2 \mu \phi+\theta}\right) .
$$

By lemma 4.4 we obtain that $r$ and $p_{\phi}$ are bounded and hence $\boldsymbol{q}_{1}$ and $\boldsymbol{q}_{2}$ are bounded.

- if $k_{1}+k_{2}=0$ and $\boldsymbol{P} \neq \mathbf{0}$ we have that the trajectories of the two particles are typically unbounded in the $(x, y)$ plane: they drift with a non-zero average velocity.

(3.2) trajectories starting and ending free but possibly spending a finite time trapped in between, leading to an asymptotic behaviour of the type "bounce-back" or "passthrough" described in points (2.1) and (2.2) above.

(3.3) trajectories starting free and making a transition to permanently trapped.

Figure 5 shows some examples. 


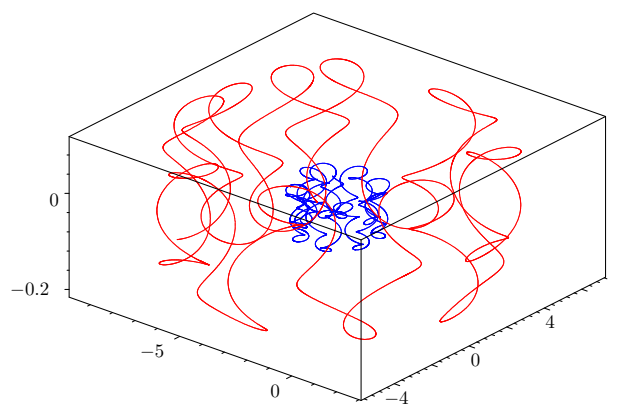

(a)

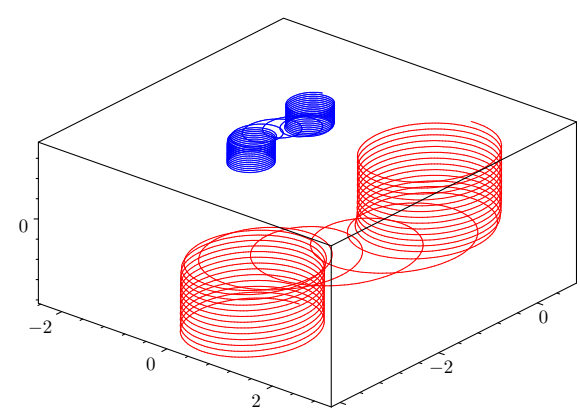

(c)

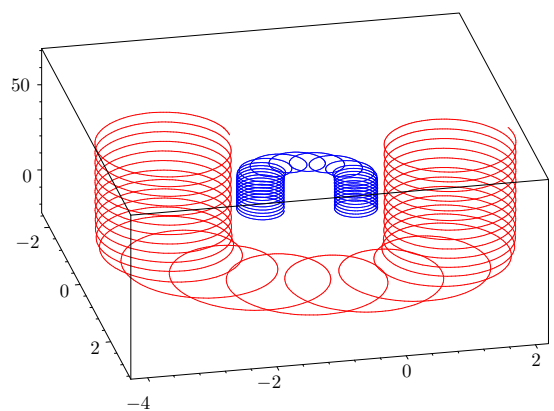

(b)

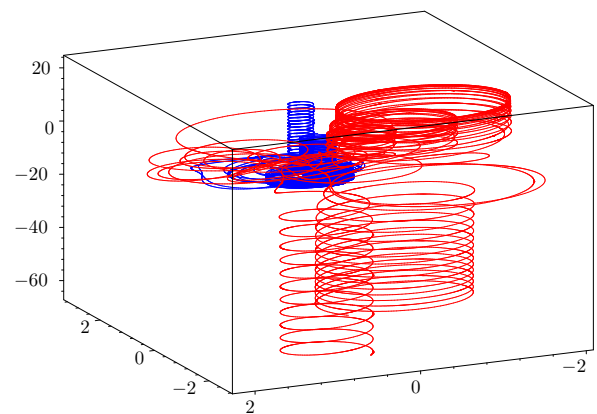

(d)

Figure 5: Four distinct dynamical behaviours. For all the figures we fix the parameters $e_{1}=$ $m_{1}=1, B=1$ and $\epsilon_{0}=0.1\left(\epsilon_{0}\right.$ is really about $8.854 \times 10^{-12} \mathrm{Fm}^{-1}$ but by choice of units/scaling symmetry we can scale it to any convenient value for the numerics) and initial conditions $q_{x_{1}}(0)=$ $-q_{x_{2}}(0)=2, p_{x_{1}}(0)=p_{x_{2}}(0)=1\left(p_{x_{1}}(0)=-p_{x_{2}}(0)=0.1\right.$ on the bottom right figure $)$ and $q_{y_{1}}(0)=q_{y_{2}}(0)=p_{y_{1}}(0)=p_{y_{2}}(0)=0$. On the top left figure we set $e_{2}=-4, m_{2}=3$ and $q_{z_{1}}(0)=-q_{z_{2}}(0)=0.1, p_{z_{1}}(0)=-p_{z_{2}}(0)=-0.01$ and obtain "atom-like" behaviour. On the top right figure we set $e_{2}=m_{2}=3$ and $q_{z_{1}}(0)=-q_{z_{2}}(0)=0.1, p_{z_{1}}(0)=-p_{z_{2}}(0)=-0.01$ and obtain "bouncing-back" behaviour. On the bottom left figure we set $e_{2}=m_{2}=3$ and $q_{z_{1}}(0)=-q_{z_{2}}(0)=10, p_{z_{1}}(0)=-p_{z_{2}}(0)=-0.85$ and obtain "pass-through" behaviour. On the bottom right figure we set $e_{2}=-4, m_{2}=3$ and $q_{z_{1}}(0)=-q_{z_{2}}(0)=10, p_{z_{1}}(0)=-p_{z_{2}}(0)=-0.2$ and obtain an orbit starting and ending free but spending a finite time trapped.

\section{THE SCATTERING MAP}

In this section we introduce the scattering map associated with the Hamiltonian system (2.1) and derive relevant properties of this map in some suitable regimes. As in section 4 , throughout this section we will consider the interaction potential to be Coulomb $V(R)=G / R$, where $R$ is the distance between the particles and $G=e_{1} e_{2} / 4 \pi \epsilon_{0}$; as before, we will also set $C_{z}=0$ and $P_{z}=0$ without loss of generality (except in section 5.3).

In the case of zero interaction, the general solution of (2.1) can be written as

$$
\boldsymbol{q}_{i}(t)=\left(\boldsymbol{R}_{i}+\boldsymbol{\rho}_{i}(t), q_{z_{i}}(t)\right), \quad \boldsymbol{p}_{i}(t)=\left(-k_{i} \mathbf{J} \boldsymbol{\rho}_{\boldsymbol{i}}(t), p_{z_{i}}\right),
$$

for $i \in\{1,2\}$, where $\boldsymbol{R}_{i}=\left(R_{x_{i}}, R_{y_{i}}\right) \in \mathbb{R}^{2}$ are the guiding centres of the particles, $\boldsymbol{\rho}_{i}(t) \in \mathbb{R}^{2}$ their gyroradii vectors, $q_{z_{i}}(t) \in \mathbb{R}$ their vertical positions and $p_{z_{i}} \in \mathbb{R}$ their vertical momenta. 
For zero interaction, $\boldsymbol{R}_{i}, \rho_{i}=\left|\boldsymbol{\rho}_{i}(t)\right|^{2} / 2$ and $p_{z_{i}}$ are conserved, while $\arg \left(\boldsymbol{\rho}_{i}(t)\right)$ rotates with gyrofrequency $\Omega_{i}$ and the vertical positions $q_{z_{i}}(t)$ evolve linearly with time.

As a consequence of theorems 3.1 and 3.3, for non-zero interactions the general solution of (2.1) can still be written in the form given in (5.1), with the variables $\boldsymbol{R}_{i}, \boldsymbol{\rho}_{i}, q_{z_{i}}$ and $p_{z_{i}}(i \in\{1,2\})$ evolving with time accordingly with the reconstruction maps and the reduced Hamiltonian systems given in the statements of theorems 3.1 and 3.3.

We will be considering the cases of section 4.3 where the dynamics in the vertical direction are unbounded: in the limits of $t \rightarrow \pm \infty$ we have that $\left|q_{z}(t)\right|=\left|q_{z_{1}}(t)-q_{z_{2}}(t)\right| \rightarrow \infty$. In such cases, the typical situation is the following: initially the particles have a large vertical separation and their trajectories are just helices of the form (5.1), as described for the zero interaction case. As their vertical separation reduces, the particles interact and their paths are no longer helices. The two particles eventually separate again and their paths approach helices again. Due to the interaction, the helices in which the particles move before and after interacting are different. The scattering map describes such asymptotic changes to the helices.

We now rigorously introduce the scattering map. We assume that as $|t| \rightarrow \infty$ the two particles have infinite vertical separation and the particles move in helices. We would like the scattering map to map the main asymptotic properties of such helices, i.e. the guiding centres and gyroradius as well as the vertical position and momentum as the particles approach $t=-\infty$, to the asymptotic properties of the helices at $t=+\infty$. We proceed as follows. First of all, we note that since $C_{z}=P_{z}=0$ from the reconstruction maps in theorems 3.1 and 3.3, we have that

$$
\begin{array}{ll}
q_{z_{1}}=\frac{m_{2}}{M} q_{z} & q_{z_{2}}=-\frac{m_{1}}{M} q_{z} \\
p_{z_{1}}=p_{z} & p_{z_{2}}=-p_{z},
\end{array}
$$

and hence it is enough to look at the asymptotics of $q_{z}$ and $p_{z}$. There are, however, some difficulties: in the limit of large vertical separation, the gyroradii of the two particles rotate uniformly with angular velocity given by the gyrofrequency of each particle and hence do not have a well-defined limit as $|t| \rightarrow \infty$. To overcome this difficulty we consider the Hamiltonian system (3.2) and introduce the following change of coordinates

$$
\boldsymbol{\rho}_{i}=\left(2 \rho_{i}\right)^{1 / 2} \mathbf{J} \boldsymbol{e}_{\theta_{i}}, \quad i \in\{1,2\},
$$

where $\boldsymbol{e}_{\theta_{i}}$ is defined in the same way as $\boldsymbol{e}_{\theta}$ in (3.7) with $\theta$ replaced by $\theta_{i}$. Note that $\rho_{i} \in \mathbb{R}$ is one half the square of the length of the gyroradius vector and $\theta_{i}$ is the $i$-th particle gyrophase. Using (5.2), we obtain the Hamiltonian system

$$
\begin{aligned}
H & =\frac{p_{z}^{2}}{2 m}+k_{1} \Omega_{1} \rho_{1}+k_{2} \Omega_{2} \rho_{2}+V(R) \\
\omega & =\sum_{i=1,2} k_{i}\left(\mathrm{~d} R_{x_{i}} \wedge \mathrm{d} R_{y_{i}}+\mathrm{d} \theta_{i} \wedge \mathrm{d} \rho_{i}\right)+\mathrm{d} q_{z} \wedge \mathrm{d} p_{z},
\end{aligned}
$$

where

$$
R=\left(\left|\boldsymbol{R}_{1}-\boldsymbol{R}_{2}+\left(2 \rho_{1}\right)^{1 / 2} \mathbf{J} \boldsymbol{e}_{\theta_{1}}-\left(2 \rho_{2}\right)^{1 / 2} \mathbf{J} \boldsymbol{e}_{\theta_{2}}\right|^{2}+q_{z}^{2}\right)^{1 / 2}
$$


The transformation (5.2) introduces two coordinate singularities at $\rho_{1}=0$ and $\rho_{2}=0$. The gyrophases $\theta_{i}$ evolve by the differential equation

$$
\dot{\theta}_{i}=\Omega_{i}+\frac{1}{k_{i}} \frac{\partial}{\partial \rho_{i}} V(R)
$$

and hence do not have well-defined limits $\theta_{i}^{ \pm}=\lim _{t \rightarrow \pm \infty} \theta_{i}(t)$. To avoid this inconvenience we introduce modified gyrophases by

$$
\phi_{i}=\theta_{i}-\Omega_{i} t, \quad i \in\{1,2\},
$$

measuring the displacement between the gyrophases in the non-zero interaction and zero interaction settings. Going to the extended phase space by introducing the conjugate variables energy $E$ and time $t$ and making the change of coordinates (5.4) we obtain the following Hamiltonian system

$$
\begin{aligned}
\mathcal{H} & =\frac{p_{z}^{2}}{2 m}+k_{1} \Omega_{1} \rho_{1}+k_{2} \Omega_{2} \rho_{2}+V(R)-E \\
\omega & =\sum_{i=1,2} k_{i}\left(\mathrm{~d} R_{x_{i}} \wedge \mathrm{d} R_{y_{i}}+\mathrm{d} \phi_{i} \wedge \mathrm{d} \rho_{i}\right)+\mathrm{d} q_{z} \wedge \mathrm{d} p_{z}+\mathrm{d}\left(E-k_{1} \Omega_{1} \rho_{1}-k_{2} \Omega_{2} \rho_{2}\right) \wedge \mathrm{d} t,
\end{aligned}
$$

where

$$
R=\left(\left|\boldsymbol{R}_{1}-\boldsymbol{R}_{2}+\left(2 \rho_{1}\right)^{1 / 2} \mathbf{J} \boldsymbol{e}_{\phi_{1}+\Omega_{1} t}-\left(2 \rho_{2}\right)^{1 / 2} \mathbf{J} \boldsymbol{e}_{\phi_{2}+\Omega_{2} t}\right|^{2}+q_{z}^{2}\right)^{1 / 2} .
$$

Note that the dynamics on level sets $\{H=E\}$ of (5.3) correspond to the dynamics of the level set $\{\mathcal{H}=0\}$ of (5.5). Introducing the asymptotic vertical energy

$$
E_{z}=E-k_{1} \Omega_{1} \rho_{1}-k_{2} \Omega_{2} \rho_{2}
$$

we obtain

$$
\begin{aligned}
\mathcal{H} & =\frac{p_{z}^{2}}{2 m}+V(R)-E_{z} \\
\omega & =\sum_{i=1,2} k_{i}\left(\mathrm{~d} R_{x_{i}} \wedge \mathrm{d} R_{y_{i}}+\mathrm{d} \phi_{i} \wedge \mathrm{d} \rho_{i}\right)+\mathrm{d} q_{z} \wedge \mathrm{d} p_{z}+\mathrm{d} E_{z} \wedge \mathrm{d} t
\end{aligned}
$$

where $R$ is given by (5.6).

Let $i \in\{1,2\}$ and let us denote by $\boldsymbol{R}_{i}^{+}, \rho_{i}^{+}, \phi_{i}^{+}$and $p_{z}^{+}$(respectively $\boldsymbol{R}_{i}^{-}, \rho_{i}^{-}, \phi_{i}^{-}$and $p_{z}^{-}$) the limits of the quantities $\boldsymbol{R}_{i}(t), \rho_{i}(t), \phi_{i}(t)$ and $p_{z}(t)$ as $t \rightarrow+\infty$ (respectively $t \rightarrow-\infty$ ). Furthermore, let us denote by $q_{z}{ }^{+}$the sign of $q_{z}(t)$ as $t \rightarrow+\infty$ and by $q_{z}{ }^{-}$the sign of $q_{z}(t)$ as $t \rightarrow-\infty$. The scattering map is the map $S: \mathbb{R}^{9} \times\{+,-\} \rightarrow \mathbb{R}^{9} \times\{+,-\}$ given by

$$
S\left(\boldsymbol{R}_{1}^{-}, \boldsymbol{R}_{2}^{-}, \rho_{1}^{-}, \rho_{2}^{-}, \phi_{1}^{-}, \phi_{2}^{-}, p_{z}^{-}, q_{z}^{-}\right)=\left(\boldsymbol{R}_{1}^{+}, \boldsymbol{R}_{2}{ }^{+}, \rho_{1}{ }^{+}, \rho_{2}{ }^{+}, \phi_{1}{ }^{+}, \phi_{2}{ }^{+}, p_{z}{ }^{+}, q_{z}{ }^{+}\right) \text {. }
$$

Remarks $\quad$ i) The scattering map is well-defined provided $\left|q_{z}(t)\right| \rightarrow \infty$ as $|t| \rightarrow \infty$.

ii) A complete study of the scattering map should take into account the asymptotic time difference for the particles to travel between two points with and without the interaction. We skip an analytic study of this asymptotic time difference since the Coulomb potential leads to unbounded time difference due to a logarithmic term. However, we report on a numerical study of the time difference in section 5.4. 
5.1. Scattering map in the limit of widely separated trajectories and rationally independent gyrofrequencies. Throughout this section we will consider that the projections of the helices on the horizontal plane in the limits $|t| \rightarrow \infty$ are two widely separated circles, i.e.

$$
\left|\rho_{1}\right|^{2}+\left|\rho_{2}\right|^{2}<<\left|\Delta_{\perp}\right|^{2}
$$

where

$$
\Delta_{\perp}=\boldsymbol{R}_{1}-\boldsymbol{R}_{2}
$$

Furthermore, we assume that the ratio of gyrofrequencies is not close to a low order rational and the relative vertical speed $\dot{q}_{z}$ is small compared to $\left|\Delta_{\perp}\right| \min \left\{\Omega_{1}, \Omega_{2}\right\}$. Then to a good approximation one can average the interaction potential over the two gyrophases (two-phase averaging, cf. [14]), obtaining the averaged Hamiltonian system:

$$
\begin{aligned}
\mathcal{H}^{e f f} & =\frac{p_{z}^{2}}{2 m}+\mathcal{V}^{e f f}-E_{z} \\
\omega & =\sum_{i=1,2} k_{i}\left(\mathrm{~d} R_{x_{i}} \wedge \mathrm{d} R_{y_{i}}+\mathrm{d} \phi_{i} \wedge \mathrm{d} \rho_{i}\right)+\mathrm{d} q_{z} \wedge \mathrm{d} p_{z}+\mathrm{d} E_{z} \wedge \mathrm{d} t,
\end{aligned}
$$

where

$$
\mathcal{V}^{e f f}=\frac{1}{4 \pi^{2}} \int_{0}^{2 \pi} \int_{0}^{2 \pi} V(R) \mathrm{d} \phi_{1} \mathrm{~d} \phi_{2}
$$

and

$$
\begin{aligned}
V(R) & =\frac{G}{R} \\
R & =\left(\left|\Delta_{\perp}\right|^{2}+2 \Delta_{\perp} \cdot \boldsymbol{w}+|\boldsymbol{w}|^{2}+q_{z}^{2}\right)^{1 / 2} \\
\boldsymbol{w} & =\left(2 \rho_{1}\right)^{1 / 2} \mathbf{J} \boldsymbol{e}_{\phi_{1}+\Omega_{1} t}-\left(2 \rho_{2}\right)^{1 / 2} \mathbf{J} \boldsymbol{e}_{\phi_{2}+\Omega_{2} t} .
\end{aligned}
$$

The average of $1 / R$ with respect to the two modified gyrophases $\phi_{1}$ and $\phi_{2}$ can be expressed most simply by change of variables of integration from $\phi_{1}$ and $\phi_{2}$ to the modulus $r$ and direction $\phi$ of $\boldsymbol{w}$. We obtain

$$
\begin{aligned}
\left\langle\frac{1}{R}\right\rangle & =\frac{1}{4 \pi^{2}} \int_{0}^{2 \pi} \int_{0}^{2 \pi} \frac{1}{R} \mathrm{~d} \phi_{1} \mathrm{~d} \phi_{2} \\
& =\frac{1}{\pi^{2}} \int_{r_{-}}^{r^{+}} \int_{0}^{2 \pi} \frac{r}{\sqrt{\left(\Delta^{2}+2 r \Delta_{\perp} . \mathbf{J} e_{\phi}+r^{2}\right)\left(r_{+}^{2}-r^{2}\right)\left(r^{2}-r_{-}^{2}\right)}} \mathrm{d} \phi \mathrm{d} r
\end{aligned}
$$

where

$$
\begin{aligned}
\Delta & =\sqrt{\left|\Delta_{\perp}\right|^{2}+q_{z}^{2}} \\
r_{ \pm} & =\left|\sqrt{2 \rho_{1}} \pm \sqrt{2 \rho_{2}}\right| .
\end{aligned}
$$


This is a type of double elliptic integral, but rather than expressing it in such terms, we choose to expand it in Taylor series to analyse the dominant effects:

$$
\begin{aligned}
& \int_{0}^{2 \pi}\left(\Delta^{2}+2 r \Delta_{\perp} . \mathbf{J} \boldsymbol{e}_{\phi}+r^{2}\right)^{-1 / 2} \mathrm{~d} \phi=\Delta^{-1} \int_{0}^{2 \pi}\left(1+\frac{2 r \Delta_{\perp} . \mathbf{J} \boldsymbol{e}_{\phi}+r^{2}}{\Delta^{2}}\right)^{-1 / 2} \mathrm{~d} \phi \\
& =\Delta^{-1} \int_{0}^{2 \pi} 1-\frac{1}{2} \frac{2 r \Delta_{\perp} . \mathbf{J} \boldsymbol{e}_{\phi}+r^{2}}{\Delta^{2}}+\frac{3}{8}\left(\frac{2 r \Delta_{\perp} . \mathbf{J} \boldsymbol{e}_{\phi}+r^{2}}{\Delta^{2}}\right)^{2} \mathrm{~d} \phi+O\left(\frac{\left|\Delta_{\perp}\right|^{4}}{\Delta^{9}}\right) \\
& =\Delta^{-1}\left(2 \pi-\pi r^{2} \frac{1}{\Delta^{2}}+3 \pi r^{2} \frac{\left|\Delta_{\perp}\right|^{2}}{\Delta^{4}}\right)+O\left(\frac{\left|\Delta_{\perp}\right|^{4}}{\Delta^{9}}\right) .
\end{aligned}
$$

Noting that

$$
\begin{aligned}
& \int_{r_{-}}^{r^{+}} \frac{r}{\sqrt{\left(r_{+}^{2}-r^{2}\right)\left(r^{2}-r_{-}^{2}\right)}} \mathrm{d} r=\frac{\pi}{2} \\
& \int_{r_{-}}^{r^{+}} \frac{r^{3}}{\sqrt{\left(r_{+}^{2}-r^{2}\right)\left(r^{2}-r_{-}^{2}\right)}} \mathrm{d} r=\frac{\pi}{4}\left(r_{+}^{2}+r_{-}^{2}\right)=\pi\left(\rho_{1}+\rho_{2}\right),
\end{aligned}
$$

and putting together (5.12) and (5.14), to second order in the ratio of gyroradii to the distance between the guiding centres, we obtain the following approximate effective potential

$$
\mathcal{V}^{e f f}=G\left(\frac{1}{\Delta}-\left(\rho_{1}+\rho_{2}\right)\left(\frac{1}{\Delta^{3}}-\frac{3}{2} \frac{\left|\Delta_{\perp}\right|^{2}}{\Delta^{5}}\right)\right),
$$

where $\Delta_{\perp}$ is given in (5.10) and $\Delta$ is given in (5.13).

Thus, we have derived an effective Hamiltonian system for the interaction of two widely separated charges in a uniform magnetic field, which is given by

$$
\begin{aligned}
\mathcal{H}^{e f f} & =\frac{p_{z}^{2}}{2 m}+\mathcal{V}^{e f f}-E_{z} \\
\omega & =\sum_{i=1,2} k_{i}\left(\mathrm{~d} R_{x_{i}} \wedge \mathrm{d} R_{y_{i}}+\mathrm{d} \phi_{i} \wedge \mathrm{d} \rho_{i}\right)+\mathrm{d} q_{z} \wedge \mathrm{d} p_{z}+\mathrm{d} E_{z} \wedge \mathrm{d} t,
\end{aligned}
$$

where $\mathcal{V}^{\text {eff }}$ is approximately given in (5.15), and we will use this approximation henceforth.

Note that the averaging procedure eliminates $t$ and implies the conservation of the quantities $\rho_{1}, \rho_{2}$ and $E_{z}$. Furthermore, the system (5.16) has a three-dimensional group of symmetries (2 translations and 1 rotation) to which correspond the following conserved quantities:

$$
\begin{aligned}
\boldsymbol{P} & =\mathbf{J}\left(k_{1} \boldsymbol{R}_{1}+k_{2} \boldsymbol{R}_{2}\right) \\
L-k_{1} \rho_{1}-k_{2} \rho_{2} & =-\frac{k_{1}}{2}\left|\boldsymbol{R}_{1}\right|^{2}-\frac{k_{2}}{2}\left|\boldsymbol{R}_{2}\right|^{2} .
\end{aligned}
$$

We will use these symmetries and conserved quantities to reduce the Hamiltonian system (5.16). We divide our analysis into two cases: $k_{1}+k_{2} \neq 0$ and $k_{1}+k_{2}=0$.

5.1.1. Case $k_{1}+k_{2} \neq 0$. From (5.17) we obtain that the centre of charge of the guiding centres

$$
\frac{k_{1} \boldsymbol{R}_{1}+k_{2} \boldsymbol{R}_{2}}{k_{1}+k_{2}}=\frac{-J \boldsymbol{P}}{k_{1}+k_{2}}
$$

is a constant. So by a translation we can assume it and $\boldsymbol{P}$ are $\mathbf{0}$. This implies the relation

$$
\boldsymbol{R}_{2}=-\frac{k_{1}}{k_{2}} \boldsymbol{R}_{1}
$$


Restricting the Hamiltonian system (5.16) to the level set determined by (5.18) gives

$$
\begin{aligned}
\mathcal{H}^{e f f} & =\frac{p_{z}{ }^{2}}{2 m}+\mathcal{V}^{e f f}-E_{z} \\
\omega & =\frac{k_{1}{ }^{2}}{e} \mathrm{~d} R_{x_{1}} \wedge \mathrm{d} R_{y_{1}}+k_{1} \mathrm{~d} \phi_{1} \wedge \mathrm{d} \rho_{1}+k_{2} \mathrm{~d} \phi_{2} \wedge \mathrm{d} \rho_{2}+\mathrm{d} q_{z} \wedge \mathrm{d} p_{z}+\mathrm{d} E_{z} \wedge \mathrm{d} t \\
\mathcal{V}^{e f f} & =\frac{G}{\left(k_{1}{ }^{2}\left|\boldsymbol{R}_{1}\right|^{2} / e^{2}+q_{z}{ }^{2}\right)^{1 / 2}}-G\left(\rho_{1}+\rho_{2}\right) \frac{q_{z}{ }^{2}-k_{1}{ }^{2}\left|\boldsymbol{R}_{1}\right|^{2} /\left(2 e^{2}\right)}{\left(k_{1}{ }^{2}\left|\boldsymbol{R}_{1}\right|^{2} / e^{2}+q_{z}\right)^{5 / 2}} .
\end{aligned}
$$

The angular momentum given in (5.17) is now given by

$$
L-k_{1} \rho_{1}-k_{2} \rho_{2}=-\frac{k_{1}^{2}}{2 e}\left|\boldsymbol{R}_{1}\right|^{2} .
$$

We introduce a corrected angular momentum

$$
p_{\theta_{R}}=L-k_{1} \rho_{1}-k_{2} \rho_{2},
$$

and note that:

- $-2 e p_{\theta_{R}}>0$,

- conservation of $p_{\theta_{R}}$ implies that the guiding centres $\boldsymbol{R}_{i}$ move in arcs of radius

$$
\sqrt{-2 e p_{\theta_{R}} / k_{i}^{2}}, \quad i \in\{1,2\} .
$$

We reduced the system by angular momentum introducing polar coordinates

$$
\boldsymbol{R}_{1}=\sqrt{-2 e p_{\theta_{R}} / k_{1}^{2}} e_{\theta_{R}}
$$

where $\boldsymbol{e}_{\theta_{R}}$ is defined in the same way as $\boldsymbol{e}_{\theta}$ in (3.7) with $\theta$ replaced by $\theta_{R}$. We note that there is a coordinate singularity when $p_{\theta_{R}}=0$ corresponding to the case when $\boldsymbol{R}_{1}=\boldsymbol{R}_{2}=0$, which is not a problem since we are dealing with the case of large $\left|\boldsymbol{R}_{1}-\boldsymbol{R}_{2}\right|$. To obtain a canonical Hamiltonian system we also introduce $p_{\phi_{i}}$ defined by

$$
p_{\phi_{i}}=k_{i} \rho_{i}=\frac{1}{2} e_{i} B\left|\boldsymbol{\rho}_{i}\right|^{2}
$$

(the quantity $e_{i} p_{\phi_{i}} / m_{i}$ is the magnetic moment of the gyrating particle) and reduce by the (extra) degree of freedom $\mathrm{d} E_{z} \wedge \mathrm{d} t$ corresponding to the coordinates in the extended phase space. We obtain the following one degree of freedom effective Hamiltonian system

$$
\begin{aligned}
H^{e f f} & =\frac{p_{z}^{2}}{2 m}+V^{e f f} \\
V^{e f f} & =\frac{G}{\left(-2 p_{\theta_{R}} / e+q_{z}^{2}\right)^{1 / 2}}-G\left(\frac{p_{\phi_{1}}}{k_{1}}+\frac{p_{\phi_{2}}}{k_{2}}\right) \frac{q_{z}^{2}+p_{\theta_{R}} / e}{\left(-2 p_{\theta_{R}} / e+q_{z}^{2}\right)^{5 / 2}} \\
\omega & =\mathrm{d} q_{z} \wedge \mathrm{d} p_{z}+\mathrm{d} \theta_{R} \wedge \mathrm{d} p_{\theta_{R}}+\mathrm{d} \phi_{1} \wedge \mathrm{d} p_{\phi_{1}}+\mathrm{d} \phi_{2} \wedge \mathrm{d} p_{\phi_{2}} .
\end{aligned}
$$

We note that assumption (5.9) implies that the effective potential in (5.21) has exactly one critical point. Furthermore, it satisfies the following conditions:

- if $G>0$, then $V^{e f f}$ is positive for all $q_{z} \in \mathbb{R}$ (see figure (6a)). It is increasing for $q_{z}<0$, decreasing for $q_{z}>0$ and has a maximum $E^{\prime}$ at $q_{z}=0$, given by

$$
E^{\prime}=\frac{G}{\left(-2 p_{\theta_{R}} / e\right)^{1 / 2}}+\frac{1}{2}\left(\frac{p_{\phi_{1}}}{k_{1}}+\frac{p_{\phi_{2}}}{k_{2}}\right) \frac{G}{\left(-2 p_{\theta_{R}} / e\right)^{3 / 2}} .
$$

Furthermore $\lim _{q_{z} \rightarrow \pm \infty} V^{e f f}=0$. 
- if $G<0$, then $V^{e f f}$ is negative for all $q_{z} \in \mathbb{R}$ (see figure (6b)). It is decreasing for $q_{z}<0$, increasing for $q_{z}>0$ and has global minimum $E^{\prime}$ (given by (5.22)) at $q_{z}=0$. Furthermore $\lim _{t \rightarrow \pm \infty} V^{e f f}=0$.

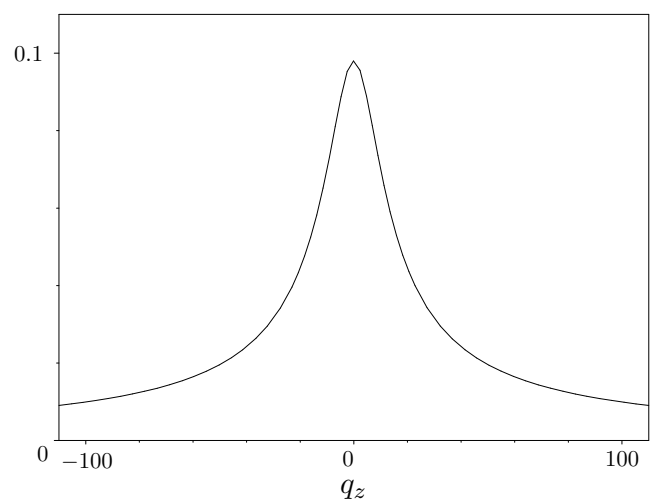

(a)

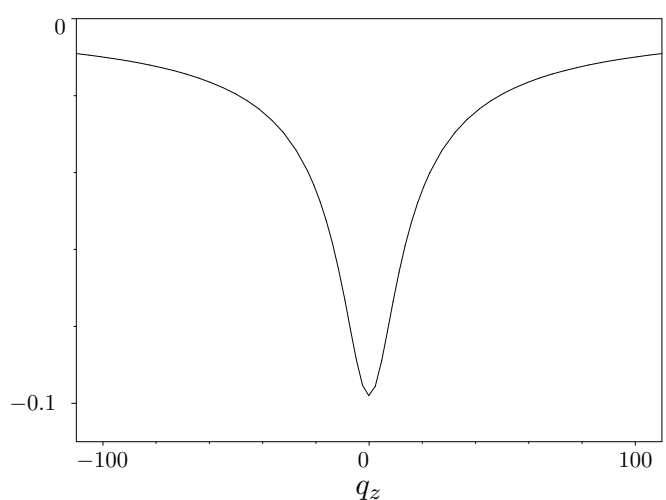

(b)

Figure 6: Plots of $V^{e f f}$. For both figures $p_{\phi_{1}} / k_{1}=p_{\phi_{2}} / k_{2}=1$ and $-2 p_{\theta_{R}} / e=100$. In the left figure $G=1$ while on the right figure $G=-1$.

The next results summarize the most relevant properties of the scattering map (5.8) for the case of two widely separated charges (satisfying $k_{1}+k_{2} \neq 0$ ) moving under the action of a uniform magnetic field and a Coulomb interaction. Before stating the results we need to introduce some notation. Given $n \in \mathbb{N}$, we define the asymptotic changes of order $n$ for $k_{1}+k_{2} \neq 0, \Gamma_{n, \gamma_{0}, \gamma_{1}, \gamma_{2}, \gamma_{3}}$, by

$$
\Gamma_{n, \gamma_{0}, \gamma_{1}, \gamma_{2}, \gamma_{3}}=\gamma_{1} \int_{\gamma_{0}}^{+\infty} \frac{1}{\left(1+x^{2}\right)^{n / 2}\left(1-U_{\gamma_{2}, \gamma_{3}}(x)\right)^{1 / 2}} \mathrm{~d} x
$$

where $U_{\gamma_{2}, \gamma_{3}}(x)$ is given by

$$
U_{\gamma_{2}, \gamma_{3}}(x)=\frac{\gamma_{2}}{\left(1+x^{2}\right)^{1 / 2}}-\gamma_{3} \frac{x^{2}-1 / 2}{\left(1+x^{2}\right)^{5 / 2}},
$$

$\gamma_{1}, \gamma_{2}$ and $\gamma_{3}$ are given by

$$
\gamma_{1}=-\frac{G e}{p_{\theta_{R}}} \sqrt{\frac{m}{2 E}}, \quad \gamma_{2}=\frac{G}{E} \sqrt{-\frac{e}{2 p_{\theta_{R}}}}, \quad \gamma_{3}=\frac{G}{E}\left(\sqrt{-\frac{e}{2 p_{\theta_{R}}}}\right)^{3}\left(\frac{p_{\phi_{1}}}{k_{1}}+\frac{p_{\phi_{2}}}{k_{2}}\right)
$$

and $\gamma_{0}$ is defined by

$$
\gamma_{0}= \begin{cases}0 & \text { if } k_{1} k_{2}>0 \text { and } E>E^{\prime} \\ q_{z}^{*} \sqrt{-e /\left(2 p_{\theta_{R}}\right)} & \text { if } k_{1} k_{2}>0 \text { and } 0<E<E^{\prime} \\ 0 & \text { if } k_{1} k_{2}<0 \text { and } E>0\end{cases}
$$

where $q_{z}{ }^{*}$ is the only positive root of $V^{e f f}\left(q_{z}\right)=E$ and $E^{\prime}$ is given by (5.22).

Theorem 5.1. Let $k_{1}+k_{2} \neq 0$ and $k_{1} k_{2}>0$ and assume that inequality (5.9) is satisfied. Then, for every level set $\left\{H^{\text {eff }}=E\right\}$ of (5.21) such that $E>0$, the scattering map $S$ is well-defined. Furthermore, there exists $E^{\prime} \in \mathbb{R}$ given by (5.22) such that:

i) for every $E>E^{\prime}$ we have that $p_{z}{ }^{+}=p_{z^{-}},\left|p_{z}{ }^{ \pm}\right|=(2 m E)^{1 / 2}$ and $q_{z}{ }^{+}=-q_{z}{ }^{-}$.

ii) for every $0<E<E^{\prime}$ we have that $p_{z}{ }^{+}=-p_{z}{ }^{-},\left|p_{z}{ }^{ \pm}\right|=(2 m E)^{1 / 2}$ and $q_{z}{ }^{+}=q_{z}{ }^{-}$. 
Let $i \in\{1,2\}$. Whenever the scattering map is well-defined, it also has the following properties:

a) there is no transfer of magnetic moment between the two particles, i.e. the gyroareas $\pi\left|\boldsymbol{\rho}_{i}\right|^{2}=2 \pi p_{\phi_{i}} / k_{i}$ are conserved and hence $\rho_{i}^{+}=\rho_{i}^{-}$.

b) the asymptotic gyrophases $\phi_{i}^{+}$and $\phi_{i}^{-}$are related by

$$
\Delta \phi_{i}=\phi_{i}^{+}-\phi_{i}^{-}=-\frac{1}{k_{i}}\left(\Gamma_{3, \gamma_{0}, \gamma_{1}, \gamma_{2}, \gamma_{3}}-\frac{3}{2} \Gamma_{5, \gamma_{0}, \gamma_{1}, \gamma_{2}, \gamma_{3}}\right) .
$$

c) the guiding centres $\boldsymbol{R}_{i}$ move in arcs of radius $\sqrt{-2 e p_{\theta_{R}} / k_{i}{ }^{2}}$ about $-\mathbf{J P} / \mu$ and rotate (about the centre $-\mathbf{J P} / \mu$ ) by an angle $\Delta \theta_{R}$ which, to leading order, is given by

$$
\Delta \theta_{R}=\frac{1}{e} \Gamma_{3, \gamma_{0}, \gamma_{1}, \gamma_{2}, \gamma_{3}} .
$$

Proof. The first part of the theorem and item a) of the second part follow trivially from an analysis of the Hamiltonian system (5.21). To prove item b) of the second part note that

$$
\Delta \phi_{i}=\phi_{i}^{+}-\phi_{i}^{-}=\int_{-\infty}^{+\infty} \frac{\partial H^{e f f}}{\partial p_{\phi_{i}}}\left(q_{z}(t), p_{z}(t)\right) \mathrm{d} t .
$$

If $E>E^{\prime}$, then

$$
\Delta \phi_{i}=\int_{-\infty}^{+\infty} \frac{\partial H^{e f f}}{\partial p_{\phi_{i}}} \frac{\mathrm{d} t}{\mathrm{~d} q_{z}} \mathrm{~d} q_{z}
$$

From (5.21), we obtain

$$
\begin{aligned}
\frac{\partial H^{e f f}}{\partial p_{\phi_{i}}} & =-\frac{G}{k_{i}} \frac{q_{z}^{2}+p_{\theta_{R}} / e}{\left(-2 p_{\theta_{R}} / e+q_{z}^{2}\right)^{5 / 2}} \\
\frac{\mathrm{d} q_{z}}{\mathrm{~d} t} & = \pm\left(\frac{2}{m}\left(E-V^{e f f}\left(q_{z}\right)\right)\right)^{1 / 2}
\end{aligned}
$$

From (5.24) and (5.25), we get

$$
\Delta \phi_{i}=-\frac{G}{k_{i}} \int_{-\infty}^{+\infty} \frac{q_{z}^{2}+p_{\theta_{R}} / e}{\left(-2 p_{\theta_{R}} / e+q_{z}^{2}\right)^{5 / 2}\left(\frac{2}{m}\left(E-V^{e f f}\left(q_{z}\right)\right)^{1 / 2}\right.} \mathrm{d} q_{z} .
$$

Making the change of variable $q_{z}=\left(-2 p_{\theta_{R}} / e\right)^{1 / 2} x$ in the integral on the right hand side of (5.26) and noting that the integrand is an even function of $q_{z}$, we obtain

$$
\Delta \phi_{i}=-\frac{\gamma_{1}}{k_{i}} \int_{0}^{+\infty} \frac{x^{2}-1 / 2}{\left(1+x^{2}\right)^{5 / 2}\left(1-\gamma_{2} /\left(1+x^{2}\right)^{1 / 2}+\gamma_{3}\left(x^{2}-1 / 2\right) /\left(1+x^{2}\right)^{5 / 2}\right)^{1 / 2}} \mathrm{~d} x,
$$

where

$$
\gamma_{1}=-\frac{G e}{p_{\theta_{R}}} \sqrt{\frac{m}{2 E}}, \quad \gamma_{2}=\frac{G}{E} \sqrt{-\frac{e}{2 p_{\theta_{R}}}}, \quad \gamma_{3}=\frac{G}{E}\left(\sqrt{-\frac{e}{2 p_{\theta_{R}}}}\right)^{3}\left(\frac{p_{\phi_{1}}}{k_{1}}+\frac{p_{\phi_{2}}}{k_{2}}\right) .
$$

The result then follows by simple manipulations. With a similar procedure, if $0<E<E^{\prime}$, we obtain

$$
\Delta \phi_{i}=-\frac{\gamma_{1}}{k_{i}} \int_{\gamma_{0}}^{+\infty} \frac{x^{2}-1 / 2}{\left(1+x^{2}\right)^{5 / 2}\left(1-\gamma_{2} /\left(1+x^{2}\right)^{1 / 2}+\gamma_{3}\left(x^{2}-1 / 2\right) /\left(1+x^{2}\right)^{5 / 2}\right)^{1 / 2}} \mathrm{~d} x,
$$

where $\gamma_{i}(i \in\{1,2,3\})$ are still given by (5.27) and $\gamma_{0}$ is given by

$$
\gamma_{0}=q_{z}^{*} \sqrt{-\frac{e}{2 p_{\theta_{R}}}}
$$


The first part of item c) follows from (5.18), (5.19) and (5.20) and the reconstruction map in theorem 3.1. To complete the proof we just need to evaluate

$$
\Delta \theta_{R}=\lim _{t \rightarrow+\infty} \theta_{R}(t)-\lim _{t \rightarrow-\infty} \theta_{R}(t)=\int_{-\infty}^{+\infty} \frac{\partial H^{e f f}}{\partial p_{\theta_{R}}}\left(q_{z}(t), p_{z}(t)\right) \mathrm{d} t
$$

Noting that

$$
\frac{\partial H^{e f f}}{\partial p_{\theta_{R}}}=\frac{G}{e\left(-2 p_{\theta_{R}} / e+q_{z}^{2}\right)^{3 / 2}}+O\left(\frac{\left(p_{\phi_{1}} / k_{1}+p_{\phi_{2}} / k_{2}\right)}{\left(-2 p_{\theta_{R}} / e+q_{z}^{2}\right)^{5 / 2}}\right),
$$

and using (5.9) we obtain that the second term on the right hand side of (5.28) is negligible. Proceeding in the same way as for the proof of item b) we obtain the required result.

Theorem 5.2. Let $k_{1}+k_{2} \neq 0$ and $k_{1} k_{2}<0$ and assume that inequality (5.9) is satisfied. Then, for every level set $\left\{H^{\text {eff }}=E\right\}$ of (5.21) such that $E>0$ the scattering map $S$ is well-defined and we have that $p_{z}^{+}=p_{z}^{-},\left|p_{z}{ }^{ \pm}\right|=(2 m E)^{1 / 2}$ and $q_{z}^{+}=-q_{z}^{-}$. Let $i \in\{1,2\}$. Whenever the scattering map is well-defined, it also has the following properties:

a) there is no transfer of magnetic moment between the two particles, i.e. the gyroareas $\pi\left|\boldsymbol{\rho}_{i}\right|^{2}=2 \pi p_{\phi_{i}} / k_{i}$ are conserved and hence $\rho_{i}^{+}=\rho_{i}^{-}$.

b) the asymptotic gyrophases $\phi_{i}^{+}$and $\phi_{i}^{-}$are related by

$$
\Delta \phi_{i}=\phi_{i}^{+}-\phi_{i}^{-}=-\frac{1}{k_{i}}\left(\Gamma_{3, \gamma_{0}, \gamma_{1}, \gamma_{2}, \gamma_{3}}-\frac{3}{2} \Gamma_{5, \gamma_{0}, \gamma_{1}, \gamma_{2}, \gamma_{3}}\right) .
$$

c) the guiding centres $\boldsymbol{R}_{i}$ move in arcs of radius $\sqrt{-2 e p_{\theta_{R}} / k_{i}{ }^{2}}$ about $-\mathbf{J P} / \mu$ and rotate (about the centre $-\mathbf{J P} / \mu$ ) by an angle $\Delta \theta_{R}$ which, to leading order, is given by

$$
\Delta \theta_{R}=\frac{1}{e} \Gamma_{3, \gamma_{0}, \gamma_{1}, \gamma_{2}, \gamma_{3}} .
$$

The proof of theorem 5.2 is identical to the proof of theorem 5.1 and so we skip it.

Remarks i) Item i) in theorem 5.1 corresponds to "pass-through" behaviour (high energy) while item ii) corresponds to "bounce-back" behaviour (small energy).

ii) In the case of two charges satisfying $k_{1}+k_{2} \neq 0$ and $k_{1} k_{2}<0$ (as in theorem 5.2) to level sets with negative energies (bounded below by $E^{\prime}$ given in (5.22)) correspond bounded motions and hence, the scattering map is not well-defined. In this case we have "atom-like" behaviour in agreement with section 4.3.

5.1.2. Case $k_{1}+k_{2}=0$. Let us consider again the Hamiltonian system (5.16). We change coordinates to

$$
\boldsymbol{C}=\frac{1}{2}\left(\boldsymbol{R}_{1}+\boldsymbol{R}_{2}\right), \quad \boldsymbol{P}=\frac{1}{2 \kappa} \mathbf{J}\left(\boldsymbol{R}_{1}-\boldsymbol{R}_{2}\right),
$$

introduce again $p_{\phi_{i}}$ defined by

$$
p_{\phi_{i}}=k_{i} \rho_{i}
$$

and reduce by the (extra) degree of freedom $\mathrm{d} E_{z} \wedge \mathrm{d} t$ corresponding to the coordinates in the extended phase space obtaining the following one degree of freedom effective Hamiltonian system

$$
\begin{aligned}
H^{\text {eff }} & =\frac{p_{z}^{2}}{2 m}+\frac{G}{\left(4 \kappa^{2}|\boldsymbol{P}|^{2}+q_{z}^{2}\right)^{1 / 2}}-G\left(\frac{p_{\phi_{1}}}{k_{1}}+\frac{p_{\phi_{2}}}{k_{2}}\right) \frac{q_{z}^{2}-2 \kappa^{2}|\boldsymbol{P}|^{2}}{\left(4 \kappa^{2}|\boldsymbol{P}|^{2}+q_{z}^{2}\right)^{5 / 2}} \\
\omega & =\mathrm{d} q_{z} \wedge \mathrm{d} p_{z}+\mathrm{d} C_{x} \wedge \mathrm{d} P_{x}+\mathrm{d} C_{y} \wedge \mathrm{d} P_{y}+\mathrm{d} \phi_{1} \wedge \mathrm{d} p_{\phi_{1}}+\mathrm{d} \phi_{2} \wedge \mathrm{d} p_{\phi_{2}} .
\end{aligned}
$$


We note that condition (5.9) implies that the effective potential in (5.30) has exactly one critical point. Furthermore, it satisfies the following condition:

- $V^{e f f}$ is negative for all $q_{z} \in \mathbb{R}$ (see figure (6b)). It is decreasing for $q_{z}<0$, increasing for $q_{z}>0$ and has global minimum $E^{\prime}$ given by

$$
E^{\prime}=\frac{G}{2|\kappa||\boldsymbol{P}|}+\frac{G\left(p_{\phi_{1}} / k_{1}+p_{\phi_{2}} / k_{2}\right)}{2(2|\kappa||\boldsymbol{P}|)^{3}}
$$

at $q_{z}=0$. Furthermore $\lim _{t \rightarrow \pm \infty} V^{e f f}=0$.

The next result summarizes the most relevant properties of the scattering map (5.8) for the case of two widely separated charges (satisfying $k_{1}+k_{2}=0$ ) moving under the action of a uniform magnetic field and a Coulomb interaction. Before stating the results we need to introduce some notation. Given, $n \in \mathbb{N}$, we define the asymptotic change of order $n$ for $k_{1}+k_{2}=0, \Gamma_{n, \gamma_{1}^{0}, \gamma_{2}^{0}, \gamma_{3}^{0}}^{0}$, by

$$
\Gamma_{n, \gamma_{1}^{0}, \gamma_{2}^{0}, \gamma_{3}^{0}}^{0}=\gamma_{1}^{0} \int_{0}^{+\infty} \frac{1}{\left(1+x^{2}\right)^{n / 2}\left(1-U_{\gamma_{2}^{0}, \gamma_{3}^{0}}(x)\right)^{1 / 2}} \mathrm{~d} x,
$$

where $U_{\gamma_{2}^{0}, \gamma_{3}^{0}}(x)$ is given by

$$
U_{\gamma_{2}^{0}, \gamma_{3}^{0}}(x)=\frac{\gamma_{2}^{0}}{\left(1+x^{2}\right)^{1 / 2}}-\gamma_{3}^{0} \frac{x^{2}-1 / 2}{\left(1+x^{2}\right)^{3 / 2}}
$$

and $\gamma_{1}^{0}, \gamma_{2}^{0}$ and $\gamma_{3}^{0}$ are given by

$$
\gamma_{1}^{0}=\frac{G}{2 \kappa^{2}|\boldsymbol{P}|^{2}} \sqrt{\frac{m}{2 E}}, \quad \gamma_{2}^{0}=\frac{G}{2|\kappa||\boldsymbol{P}| E}, \quad \gamma_{3}^{0}=\frac{G}{8|\kappa|^{3}|\boldsymbol{P}|^{3} E}\left(\frac{p_{\phi_{1}}}{k_{1}}+\frac{p_{\phi_{2}}}{k_{2}}\right) .
$$

Theorem 5.3. Let $k_{1}+k_{2}=0$ and assume that inequality (5.9) is satisfied. Then, for every level set $\left\{H^{e f f}=E\right\}$ of (5.30) such that $E>0$ the scattering map $S$ is well-defined and we have that $p_{z}^{+}=p_{z}^{-},\left|p_{z}{ }^{ \pm}\right|=(2 m E)^{1 / 2}$ and $q_{z}^{+}=-q_{z}^{-}$. Let $i \in\{1,2\}$. Whenever the scattering map is well-defined, it also has the following properties:

a) there is no transfer of magnetic moment between the two particles, i.e. the gyroareas $\pi\left|\boldsymbol{\rho}_{i}\right|^{2}=2 \pi p_{\phi_{i}} / k_{i}$ are conserved and hence $\rho_{i}^{+}=\rho_{i}^{-}$.

b) the asymptotic gyrophases $\phi_{i}^{+}$and $\phi_{i}^{-}$are related by

$$
\Delta \phi_{i}=\phi_{i}^{+}-\phi_{i}^{-}=-\frac{1}{k_{i}}\left(\Gamma_{3, \gamma_{1}^{0}, \gamma_{2}^{0}, \gamma_{3}^{0}}^{0}-\frac{3}{2} \Gamma_{5, \gamma_{1}^{0}, \gamma_{2}^{0}, \gamma_{3}^{0}}^{0}\right)
$$

c) the distance between the guiding centres is conserved:

$$
\left|\boldsymbol{R}_{1}{ }^{+}-\boldsymbol{R}_{2}{ }^{+}\right|=\left|\boldsymbol{R}_{1}{ }^{-}-\boldsymbol{R}_{2}{ }^{-}\right|=2|\kappa||\boldsymbol{P}| .
$$

d) to leading order, the asymptotic guiding centres $\boldsymbol{R}_{i}^{+}$and $\boldsymbol{R}_{i}^{-}$are related by

$$
\boldsymbol{R}_{i}^{+}-\boldsymbol{R}_{i}^{-}=-4 \kappa^{2} \Gamma_{3, \gamma_{1}^{0}, \gamma_{2}^{0}, \gamma_{3}^{0}} \boldsymbol{P}
$$

Proof. The first part of the theorem and item i) of the second part follow trivially from an analysis of the Hamiltonian system (5.30). The proof of item b) is analogous to the proof of item b) of theorem 5.1. Item c) follows from (5.29). To prove item d) we invert (5.29) to obtain

$$
\boldsymbol{R}_{1}=\boldsymbol{C}-\kappa \mathbf{J} \boldsymbol{P}, \quad \boldsymbol{R}_{2}=\boldsymbol{C}+\kappa \mathbf{J} \boldsymbol{P}
$$


Noting that

$$
\boldsymbol{R}_{i}^{+}-\boldsymbol{R}_{i}^{-}=\lim _{t \rightarrow+\infty} \boldsymbol{C}(t)-\lim _{t \rightarrow-\infty} \boldsymbol{C}(t)=\int_{-\infty}^{+\infty} \frac{\partial H^{e f f}}{\partial \boldsymbol{P}}\left(q_{z}(t), p_{z}(t)\right) \mathrm{d} t
$$

and proceeding in the same way as we did for the proof of item c) of theorem 5.1 we obtain the result.

Remark In the setting of theorem 5.3, to level sets with negative energies (bounded below by $E^{\prime}$ given in (5.31)) correspond bounded motions and the scattering map is not defined. In such energy regimes the dynamics exhibit "atom-like" behaviour in agreement with section 4.3. Otherwise, on level sets with positive energies the dynamics exhibit "pass-through" behaviour.

We should note that theorems 5.1, 5.2 and 5.3 apply only to the averaged systems (5.21) and (5.30); the error of approximation caused by the derivation of such Hamiltonian systems has not been analysed in detail.

\subsection{Scattering map in the limit of widely separated trajectories and rationally de-} pendent gyrofrequencies. As in the previous section, we will consider that the projections of the helices on the horizontal plane in the limits $|t| \rightarrow \infty$ are two widely separated circles satisfying (5.9). Furthermore, we assume that the particles' gyrofrequencies have ratio close to a low order rational. Then correlations between the gyrophases may lead to corrections to the scattering map obtained in the previous section, of the order of the ratio between a gyroradius and the horizontal distance between guiding centres. In particular, although one combination of the magnetic moments is still an adiabatic invariant and it happens to imply that the vertical kinetic energy is approximately unchanged by the interaction, there can be transfer of horizontal kinetic energy between the particles.

To analyse this case, let $\lambda_{1}, \lambda_{2} \in \mathbb{Z}$ be relatively prime integers such that $\lambda_{1} \Omega_{1}+\lambda_{2} \Omega_{2}=0$ and introduce the near-resonant combination $\phi$ of the gyrophases and a complementary one $\psi$ by

$$
\phi=\lambda_{1} \theta_{1}+\lambda_{2} \theta_{2}, \quad \psi=\gamma_{1} \theta_{1}+\gamma_{2} \theta_{2}
$$

where $\gamma_{1}, \gamma_{2} \in \mathbb{Z}$ are relatively prime integers such that $\lambda_{1} \gamma_{2}-\lambda_{2} \gamma_{1}=1$. Introducing conjugate momenta $p_{\phi}$ and $p_{\psi}$, given by

$$
p_{\phi}=\gamma_{2} k_{1} \rho_{1}-\gamma_{1} k_{2} \rho_{2}, \quad p_{\psi}=\lambda_{1} k_{2} \rho_{2}-\lambda_{2} k_{1} \rho_{1}
$$

and substituting the gyrophases $\theta_{1}, \theta_{2}$ and their conjugate momenta $\rho_{1}, \rho_{2}$ by the new variables introduced above in the Hamiltonian system (5.3), we obtain

$$
\begin{aligned}
H & =\frac{p_{z}^{2}}{2 m}+\left(\gamma_{1} \Omega_{1}+\gamma_{2} \Omega_{2}\right) p_{\psi}+V(R) \\
\omega & =\sum_{i=1,2} k_{i}\left(\mathrm{~d} R_{x_{i}} \wedge \mathrm{d} R_{y_{i}}\right)+\mathrm{d} \phi \wedge \mathrm{d} p_{\phi}+\mathrm{d} \psi \wedge \mathrm{d} p_{\psi}+\mathrm{d} q_{z} \wedge \mathrm{d} p_{z}
\end{aligned}
$$


where $R=\left(|\boldsymbol{w}|^{2}+2 \Delta_{\perp} \cdot \boldsymbol{w}+\Delta^{2}\right)^{1 / 2}$ and

$$
\begin{aligned}
\Delta_{\perp} & =\boldsymbol{R}_{1}-\boldsymbol{R}_{2} \\
\Delta & =\sqrt{\left|\Delta_{\perp}\right|^{2}+q_{z}^{2}} \\
\boldsymbol{w} & =\left(\frac{2}{k_{1}}\left(\lambda_{1} p_{\phi}+\gamma_{1} p_{\psi}\right)\right)^{1 / 2} \mathbf{J} \boldsymbol{e}_{\gamma_{2} \phi-\lambda_{2} \psi}-\left(\frac{2}{k_{2}}\left(\lambda_{2} p_{\phi}+\gamma_{2} p_{\psi}\right)\right)^{1 / 2} \mathbf{J} \boldsymbol{e}_{\lambda_{1} \psi-\gamma_{1} \phi} .
\end{aligned}
$$

Then one can average the Hamiltonian system (5.32) with respect to $\psi$, obtaining the following averaged Hamiltonian system:

$$
\begin{aligned}
H^{e f f} & =\frac{p_{z}^{2}}{2 m}+\left(\gamma_{1} \Omega_{1}+\gamma_{2} \Omega_{2}\right) p_{\psi}+V^{e f f} \\
\omega & =\sum_{i=1,2} k_{i}\left(\mathrm{~d} R_{x_{i}} \wedge \mathrm{d} R_{y_{i}}\right)+\mathrm{d} \phi \wedge \mathrm{d} p_{\phi}+\mathrm{d} \psi \wedge \mathrm{d} p_{\psi}+\mathrm{d} q_{z} \wedge \mathrm{d} p_{z},
\end{aligned}
$$

where

$$
V^{e f f}=\frac{1}{2 \pi} \int_{0}^{2 \pi} V(R) \mathrm{d} \psi .
$$

In particular, adiabatic invariance of $p_{\psi}$ and conservation of $H^{\text {eff }}$ imply that the vertical kinetic energy is unchanged by the interaction.

To examine whether there is some transfer of horizontal kinetic energy, we proceed in the same way as in section 5.1, i.e. we expand the interaction potential $V(R)$ in Taylor's series, neglect terms of order $O\left(\left|\Delta_{\perp}\right| / \Delta^{5}\right)$ and integrate the remaining terms with respect to $\psi$. If $\left|\lambda_{1}\right|+2\left|\lambda_{2}\right| \neq 0$ or $2\left|\lambda_{1}\right|+\left|\lambda_{2}\right| \neq 0$ there are no terms of order $O\left(\left|\Delta_{\perp}\right| / \Delta^{5}\right)$ and we neglect terms of order $O\left(1 / \Delta^{5}\right)$. We separate our analysis into three cases: $\left|\lambda_{1}\right| \neq\left|\lambda_{2}\right|, \lambda_{1}-\lambda_{2}=0$ and $\lambda_{1}+\lambda_{2}=0$.

5.2.1. Case $\left|\lambda_{1}\right| \neq\left|\lambda_{2}\right|$. Applying the averaging procedure described above, we obtain the following effective Hamiltonian system:

$$
\begin{aligned}
H^{e f f} & =\frac{p_{z}^{2}}{2 m}+\left(\gamma_{1} \Omega_{1}+\gamma_{2} \Omega_{2}\right) p_{\psi}+V^{e f f} \\
\omega & =\sum_{i=1,2} k_{i}\left(\mathrm{~d} R_{x_{i}} \wedge \mathrm{d} R_{y_{i}}\right)+\mathrm{d} \phi \wedge \mathrm{d} p_{\phi}+\mathrm{d} \psi \wedge \mathrm{d} p_{\psi}+\mathrm{d} q_{z} \wedge \mathrm{d} p_{z},
\end{aligned}
$$

where

$$
V^{e f f}=G\left(\frac{1}{\Delta}-\left(\rho_{1}+\rho_{2}\right)\left(\frac{1}{\Delta^{3}}-\frac{3}{2} \frac{\left|\Delta_{\perp}\right|^{2}}{\Delta^{5}}\right)\right) .
$$

and

$$
\begin{aligned}
\Delta_{\perp} & =\boldsymbol{R}_{1}-\boldsymbol{R}_{2} \\
\Delta & =\sqrt{\left|\Delta_{\perp}\right|^{2}+q_{z}{ }^{2}} \\
\rho_{1}+\rho_{2} & =\left(\frac{\lambda_{1}}{k_{1}}+\frac{\lambda_{2}}{k_{2}}\right) p_{\phi}+\left(\frac{\gamma_{1}}{k_{1}}+\frac{\gamma_{2}}{k_{2}}\right) p_{\psi} .
\end{aligned}
$$

Since $V^{e f f}$ is independent of $\phi$ (up to an error of order $O\left(\left|\Delta_{\perp}\right| / \Delta^{5}\right)$ if $\left|\lambda_{1}\right|+2\left|\lambda_{2}\right|=0$ or $2\left|\lambda_{1}\right|+\left|\lambda_{2}\right|=0$ or of order $O\left(1 / \Delta^{5}\right)$ otherwise) we obtain that $p_{\phi}$ is also approximately conserved, and then the analysis reduces to the cases of sections 5.1.1 and 5.1.2. In particular, integrating the error bound along unperturbed trajectories shows that the effect of the errors 
is of order at most $\left|\Delta_{\perp}\right|^{-3}$ if $\left|\lambda_{1}\right|+2\left|\lambda_{2}\right|=0$ or $2\left|\lambda_{1}\right|+\left|\lambda_{2}\right|=0$ or of order at most $\left|\Delta_{\perp}\right|^{-4}$ otherwise.

We should remark, however, that effects of resonance would be noticeable if we had considered higher order terms in the Taylor's series expansions of the interaction potential $V(R)$.

5.2.2. Case $\lambda_{1}-\lambda_{2}=0$. We start by noticing that $\lambda_{1}-\lambda_{2}=0$ is equivalent to $\Omega_{1}+\Omega_{2}=0$ and that, without loss of generality, one can can fix $\lambda_{1}, \lambda_{2}, \gamma_{1}, \gamma_{2} \in \mathbb{Z}$ so that $\lambda_{1}=1, \lambda_{2}=1$ and $\gamma_{2}-\gamma_{1}=1$. Then, applying the averaging procedure described above, we obtain an effective Hamiltonian system of the form (5.33) with effective potential $V^{e f f}$ given by

$$
\begin{aligned}
H^{e f f} & =\frac{p_{z}^{2}}{2 m}-\Omega p_{\psi}+V^{e f f} \\
\omega & =\sum_{i=1,2} k_{i}\left(\mathrm{~d} R_{x_{i}} \wedge \mathrm{d} R_{y_{i}}\right)+\mathrm{d} \phi \wedge \mathrm{d} p_{\phi}+\mathrm{d} \psi \wedge \mathrm{d} p_{\psi}+\mathrm{d} q_{z} \wedge \mathrm{d} p_{z} \\
V^{e f f} & =G\left(\frac{1}{\Delta}-\left(\rho_{1}+\rho_{2}\right)\left(\frac{1}{\Delta^{3}}-\frac{3}{2} \frac{\left|\Delta_{\perp}\right|^{2}}{\Delta^{5}}\right)+3 \sqrt{\rho_{1} \rho_{2}} \frac{\left(\Delta_{\perp_{y}}^{2}-\Delta_{\perp x}^{2}\right) \cos (\phi)-2 \Delta_{\perp x} \Delta_{\perp y} \sin (\phi)}{\Delta^{5}}\right),
\end{aligned}
$$

where $\Omega=\Omega_{1}=-\Omega_{2}, \Delta_{\perp}=\left(\Delta_{\perp x}, \Delta_{\perp y}\right), \Delta_{\perp}$ and $\Delta$ are as given in (5.34) and

$$
\rho_{1}=\frac{\lambda_{1} p_{\phi}+\gamma_{1} p_{\psi}}{k_{1}}, \quad \rho_{2}=\frac{\lambda_{2} p_{\phi}+\gamma_{2} p_{\psi}}{k_{2}} .
$$

We can now use the system symmetries as in sections 5.1.1 and 5.1.2 to reduce the Hamiltonian system given by (5.35) to one with two degrees of freedom. We skip such details here and proceed to state results which are analogous to those of sections 5.1.1 and 5.1.2, but since $p_{\phi}$ is not necessarily conserved there is no longer any reason to expect the magnetic moments of the particles to be individually conserved and indeed we prove typical transfer of horizontal kinetic energy between the particles.

Theorem 5.4. Let $k_{1}+k_{2} \neq 0$ and assume that inequality (5.9) is satisfied. Then, for every level set $\left\{H^{e f f}=E\right\}$ of (5.35) such that $E>-\Omega p_{\psi}$ the scattering map $S$ is well-defined and we have that $p_{z}^{+}=p_{z}^{-},\left|p_{z}{ }^{ \pm}\right|=\left(2 m\left(E+\Omega p_{\psi}\right)\right)^{1 / 2}$ and $q_{z}^{+}=-q_{z}^{-}$. Let $i \in\{1,2\}$. Whenever the scattering map is well-defined, it also has the following properties:

a) the difference $p_{\psi}$ in magnetic moments is approximately conserved.

b) typically there is transfer of horizontal kinetic energy between the particles.

c) the distances of the guiding centres from $-\mathbf{J P} / \mu$ typically change, but conserve $L=$ $k_{1} \rho_{1}+k_{2} \rho_{2}-\frac{k_{1}}{2}\left|\boldsymbol{R}_{1}\right|^{2}-\frac{k_{2}}{2}\left|\boldsymbol{R}_{2}\right|^{2}$ and $-\mathbf{J} \boldsymbol{P}=k_{1} \boldsymbol{R}_{1}+k_{2} \boldsymbol{R}_{2}$

Proof. Most of the proof is analogous to that for theorems 5.1 and 5.2. For part b), we use

$$
\Delta p_{\phi}=-\int V^{\prime}(\phi) \mathrm{d} t
$$

and note that to first order in $G$ this can be evaluated along unperturbed orbits, i.e. $\rho_{j}$ and $\Delta_{\perp}$ constant and $\Delta=\sqrt{\left|\Delta_{\perp}\right|^{2}+v^{2} t^{2}}$ for some relative vertical speed $v$. Thus to first order in $G$,

$$
\Delta p_{\phi}=G(A \sin \phi+B \cos \phi)
$$

with

$$
A=3 \sqrt{\rho_{1} \rho_{2}}\left(\Delta_{\perp y}^{2}-\Delta_{\perp x}^{2}\right) \int\left(\left|\Delta_{\perp}\right|^{2}+v^{2} t^{2}\right)^{-5 / 2} \mathrm{~d} t
$$


and similar for $B$. Evaluating the integral in the expression for $A$, we obtain

$$
A=\frac{4 \sqrt{\rho_{1} \rho_{2}}\left(\Delta_{\perp y}^{2}-\Delta_{\perp x}^{2}\right)}{v\left|\Delta_{\perp}\right|^{4}}, \quad B=\frac{8 \sqrt{\rho_{1} \rho_{2}}\left(\Delta_{\perp x} \Delta_{\perp y}\right)}{v\left|\Delta_{\perp}\right|^{4}},
$$

which shows the effect on $p_{\phi}$ to be of order $1 /\left|\Delta_{\perp}\right|^{2}$.

In particular, for small $G \neq 0$ it is not constant as a function of $\phi$. Now $\Delta p_{\phi}$ is analytic in the initial conditions and parameters, so its zeroes form isolated codimension-one subsets. Thus typically $\Delta p_{\phi}$ is non-zero. This means that there is transfer of horizontal kinetic energy between the particles. The magnitude for small $G$ (which is equivalent to large $\Delta_{\perp}$ ) is as above.

Theorem 5.5. Let $k_{1}+k_{2}=0$ and assume that inequality (5.9) is satisfied. Then, for every level set $\left\{H^{e f f}=E\right\}$ of (5.35) such that $E>-\Omega p_{\psi}$ the scattering map $S$ is well-defined and we have that $p_{z}^{+}=p_{z}^{-},\left|p_{z}{ }^{ \pm}\right|=\left(2 m\left(E+\Omega p_{\psi}\right)\right)^{1 / 2}$ and $q_{z}^{+}=-q_{z}^{-}$. Let $i \in\{1,2\}$. Whenever the scattering map is well-defined, it also has the following properties:

a) the difference $p_{\psi}$ in magnetic moments is approximately conserved.

b) typically there is transfer of horizontal kinetic energy between the particles.

c) the positions of the guiding centres typically move, but conserve $k_{1}\left(\boldsymbol{R}_{1}-\boldsymbol{R}_{2}\right)=\boldsymbol{P}$ and $L / k_{1}=\rho_{1}-\rho_{2}-\left|\boldsymbol{R}_{1}\right|^{2} / 2+\left|\boldsymbol{R}_{2}\right|^{2} / 2$.

Proof. Similar to theorem 5.4.

5.2.3. $\lambda_{1}+\lambda_{2}=0$. Note that $\lambda_{1}+\lambda_{2}=0$ is equivalent to $\Omega_{1}=\Omega_{2}$. Without loss of generality, one can can fix $\lambda_{1}, \lambda_{2}, \gamma_{1}, \gamma_{2} \in \mathbb{Z}$ so that $\lambda_{1}=-\lambda_{2}=1$ and $\gamma_{2}+\gamma_{1}=1$. Applying the averaging procedure described at the beginning of section 5.2, we will obtain that the total magnetic moment $p_{\psi}=k_{1} \rho_{1}+k_{2} \rho_{2}$ is an adiabatic invariant. Under the adiabatic approximation, the vertical kinetic energy is unchanged by the interaction and we prove there is typically a transfer of horizontal kinetic energy between the particles, as in the previous case. In this case, however, we will then go beyond the adiabatic approximation, using the exceptional additional invariant $W$ to reduce the scattering problem to two degrees of freedom. In particular we will refine the qualitative description obtained in section 4.3.1 for this case, and prove that there is also typically a transfer between horizontal and vertical kinetic energy.

Using the averaging approximation, we obtain an effective Hamiltonian system of the form (5.33) with effective potential $V^{e f f}$ given by

$$
\begin{aligned}
H^{e f f} & =\frac{p_{z}^{2}}{2 m}+\Omega p_{\psi}+V^{e f f} \\
\omega & =\sum_{i=1,2} k_{i}\left(\mathrm{~d} R_{x_{i}} \wedge \mathrm{d} R_{y_{i}}\right)+\mathrm{d} \phi \wedge \mathrm{d} p_{\phi}+\mathrm{d} \psi \wedge \mathrm{d} p_{\psi}+\mathrm{d} q_{z} \wedge \mathrm{d} p_{z} \\
V^{e f f} & =G\left(\frac{1}{\Delta}-\left(\rho_{1}+\rho_{2}-2 \sqrt{\rho_{1} \rho_{2}} \cos (\phi)\right)\left(\frac{1}{\Delta^{3}}-\frac{3}{2} \frac{\left|\Delta_{\perp}\right|^{2}}{\Delta^{5}}\right)\right),
\end{aligned}
$$

where $\Omega=\Omega_{1}=\Omega_{2}, \Delta_{\perp}=\left(\Delta_{\perp_{x}}, \Delta_{\perp y}\right), \Delta_{\perp}$ and $\Delta$ are as given in (5.34) and

$$
\rho_{1}=\frac{\lambda_{1} p_{\phi}+\gamma_{1} p_{\psi}}{k_{1}}, \quad \rho_{2}=\frac{\lambda_{2} p_{\phi}+\gamma_{2} p_{\psi}}{k_{2}} .
$$

Theorem 5.6. Let $\Omega_{1}=\Omega_{2}=\Omega$ and assume that inequality (5.9) is satisfied. Then, for every level set $\left\{H^{\text {eff }}=E\right\}$ of (5.36) such that $E>\Omega p_{\psi}$ the scattering map $S$ is well-defined and has the following properties: 
a) the total magnetic moment $p_{\psi}$ is approximately conserved.

b) typically there is transfer of horizontal kinetic energy between the particles.

c) the distances of the guiding centres from $-\mathbf{J P} / \mu$ typically change, but conserve $p_{\psi}-L=$ $\frac{k_{1}}{2}\left|\boldsymbol{R}_{1}\right|^{2}+\frac{k_{2}}{2}\left|\boldsymbol{R}_{2}\right|^{2}$ and $-\mathbf{J} \boldsymbol{P}=k_{1} \boldsymbol{R}_{1}+k_{2} \boldsymbol{R}_{2}$

Proof. The analysis is very similar to that for theorem 5.4.

Next, as promised, the extra conserved quantity $W$ of proposition 2.1 enables us to push the analysis beyond the adiabatic approximation in this case. We perform exact reduction using $W$, obtaining the two degrees of freedom reduced Hamiltonian system of corollary 3.2 , given by

$$
\begin{aligned}
H & =\frac{1}{2 m}\left(p_{r}{ }^{2}+p_{z}{ }^{2}\right)+\frac{A^{2}}{r^{2}}+B^{2} r^{2}+E_{c}+V(R) \\
\omega & =\mathrm{d} r \wedge \mathrm{d} p_{r}+\mathrm{d} q_{z} \wedge \mathrm{d} p_{z}
\end{aligned}
$$

where

$$
A^{2}=\frac{1}{2 m}\left(\frac{2 \mu p_{\theta}-W}{2 \mu}\right)^{2}, \quad B^{2}=\frac{e^{2}}{8 m}, \quad E_{c}=\frac{e}{2 m}\left(p_{\theta}+\frac{W}{2 \mu}\right)+\frac{1}{2 M} P_{z}^{2}
$$

and $V(R)$ is the Coulomb potential $V(R)=G / R$ with $R=\left(r^{2}+q_{z}^{2}\right)^{1 / 2}$.

We obtain the following result, refining that of section 4.3.1.

Proposition 5.7. The Hamiltonian system (5.37) has the following properties:

i) The Hamiltonian function $H$ is bounded below by $E_{c}+2 A B$.

ii) It has a unique equilibrium at $r=r^{*}, p_{r}=q_{z}=p_{z}=0$, where $r^{*}$ is the unique positive root of the equation

$$
\frac{2 A^{2}}{r^{3}}-2 B^{2} r+\frac{G}{r}=0 .
$$

Furthermore, the equilibrium is a saddle with eigenvalues

$$
\pm \sqrt{\frac{G}{m r^{* 3}}}, \quad \pm \mathrm{i} \sqrt{\frac{2\left(3 A^{2}+B^{2} r^{* 4}+G r^{*}\right)}{m r^{* 4}}} .
$$

iii) Let $E^{*}=A^{2} / r^{* 2}+B^{2} r^{* 2}+E_{c}$ be the energy of the level set of (5.37) containing the equilibrium. Then, the Hamiltonian system (5.37) possesses a family of hyperbolic periodic orbits $\gamma_{E}$ parametrized by the energy $E>E^{*}$ and given by

$$
\frac{1}{2 m} p_{r}^{2}+\frac{A^{2}}{r^{2}}+B^{2} r^{2}+\frac{G}{R}=E-E_{c} .
$$

iv) The stable manifold of the periodic orbit separates incoming orbits into pass-through and bounce-back orbits. The flux of energy-surface volume over the saddle (i.e. pass-through orbits) for energy $E>E^{*}$ is given by

$$
2\left(r^{+}(E)-r^{-}(E)\right) \sqrt{2 m\left(E-E_{c}\right)} \int_{0}^{1}(1-U(z))^{1 / 2} \mathrm{~d} z
$$

where $0<r^{-}(E)<r^{+}(E)$ are the two positive roots of $A^{2} / r^{2}+B^{2} r^{2}+G / r=E-E_{c}$ and

$$
\begin{aligned}
U(z) & =\frac{A^{2}-B^{2} r^{4}(z)-G r(z)}{2 m\left(E-E_{c}\right) r^{2}(z)} \\
r(z) & =r^{-}(E)+\left(r^{+}(E)-r^{-}(E)\right) z .
\end{aligned}
$$


Proof. The proof of the first two items is trivial and we skip it. For the proof of the third item we note that $q_{z}=p_{z}=0$ is an invariant plane under the dynamics defined by (5.37). Furthermore, the restriction of (5.37) to $q_{z}=p_{z}=0$ defines a one degree of freedom Hamiltonian system given by

$$
\begin{aligned}
H & =\frac{1}{2 m} p_{r}^{2}+\frac{A^{2}}{r^{2}}+B^{2} r^{2}+E_{c}+\frac{G}{R} \\
\omega & =\mathrm{d} r \wedge \mathrm{d} p_{r} .
\end{aligned}
$$

This system is the reduced Hamiltonian system corresponding to the interaction of two charges moving in a plane under the action of a magnetic field in the particular case of equal gyrofrequencies $\Omega_{1}=\Omega_{2}$ and has been extensively studied in [19]. In particular, from the results in [19] it follows that the Hamiltonian system (5.38) has a unique (elliptic) equilibrium corresponding to the level set of energy $E=E^{*}$ and for every $E>E^{*}$ the level set of energy $E$ is composed of a periodic orbit $\tilde{\gamma}_{E}=\left(r_{E}(t), p_{r}(t)\right)$ of period $T(E)>0$, given by

$$
\frac{1}{2 m} p_{r}^{2}+\frac{A^{2}}{r^{2}}+B^{2} r^{2}+\frac{G}{r}=E-E_{c} .
$$

Furthermore, for every $E>E^{*}$ there exist positive values $0<r^{-}(E)<r^{+}(E)$ such that for all $0<t<T(E)$ we have that $r^{-}(E) \leq r_{E}(t) \leq r^{+}(E)$. Therefore, the full system (5.37) possesses a family of periodic orbits $\gamma_{E}=\left(r_{E}(t), p_{r E}(t), 0,0\right)$ parametrized by the energy $E>E^{*}$.

To see that the periodic orbits are hyperbolic we construct a forward invariant conefield with a uniform expansion estimate. Let $\left(\delta_{r}, \delta_{p_{r}}, \delta_{q_{z}}, \delta_{p_{z}}\right)$ denote a tangent vector to the phase space of (5.37). Then, for $E>E^{*}$ the linearized dynamics around a periodic orbit of the family $\gamma_{E}$ are given by the following system of differential equations

$$
\begin{aligned}
\dot{\delta}_{q_{z}} & =\frac{1}{m} \delta_{p_{z}}, & & \dot{\delta}_{p_{z}}=\frac{G}{\left(r_{E}(t)\right)^{3}} \delta_{q_{z}} \\
\dot{\delta}_{r} & =\frac{1}{m} \delta_{p_{r}}, & \dot{\delta}_{p_{r}} & =-\frac{2\left(3 A^{2}+B^{2}\left(r_{E}(t)\right)^{4}+G r_{E}(t)\right)}{\left(r_{E}(t)\right)^{4}} \delta_{p_{z}} .
\end{aligned}
$$

Thus the cone $\delta p_{z} \delta q_{z}>0$ is forward invariant. Furthermore it is absorbed in bounded time by the subcone $\sqrt{A} \leq \delta p_{z} / \delta q_{z} \leq \sqrt{B}$ for any $A<\min _{t} m G / r_{E}(t)^{3}, B>\max _{t} m G / r_{E}(t)^{3}$. In this subcone,

$$
\frac{\mathrm{d}}{\mathrm{d} t}\left(\delta p_{z}^{2}+\delta q_{z}^{2}\right)=2\left(\frac{1}{m}+\frac{G}{r^{3}}\right) \delta p_{z} \delta q_{z} \geq 2 \min _{t}\left(\frac{1}{m}+\frac{G}{r_{E}(t)^{3}}\right) \frac{\sqrt{A}}{1+B}\left(\delta q_{z}^{2}+\delta p_{z}^{2}\right),
$$

giving a uniform expansion estimate. The $\left(r, p_{r}\right)$ directions are not relevant because they correspond to the flow direction and change of energy.

The proof of item (iv) follows by a result in [15], stating that the flux of energy-surface volume across a surface $S$ in an energy surface for a two degrees of freedom Hamiltonian system is the action integral of its boundary $S$. In our particular case the boundary is the hyperbolic periodic orbit of item iii). Thus, the result is obtained by computing the action integral along such orbit.

The motion in $\left(r, p_{r}\right)$ for frozen $q_{z}$ is oscillatory with frequency near $\Omega_{1}=\Omega_{2}=\Omega$. Indeed if the contribution of $V(R)$ is neglected, the solutions are $r^{2}=C+D \cos \left(\Omega\left(t-t_{0}\right)\right)$ with $C^{2}-D^{2}=A^{2} / B^{2}$. Under the conditions of widely separated helices or small $p_{z}$, one can average with respect to this oscillation, obtaining an adiabatic invariant for it and an effective 
Hamiltonian for the $\left(q_{z}, p_{z}\right)$ motion. Up to scale, the adiabatic invariant is the total magnetic moment $k_{1} \rho_{1}+k_{2} \rho_{2}$, just as we obtained without the reduction by $W$. We could also use the reconstruction of theorem 3.9 to reveal that there is in general a transfer of horizontal kinetic energy between the particles, equivalently of magnetic moment, as already obtained. Conservation of $W$ and total magnetic moment $k_{1} \rho_{1}+k_{2} \rho_{2}$ leave a 2 -torus of possible values for $\left(\rho_{1}, \rho\right)$ and in general their lengths change as a result of the interaction.

Instead of pursuing this line, we will treat the exact reduced system (i.e. without averaging), and show that there is typically also some exchange between the vertical and horizontal kinetic energies. Let $H_{v e r}^{ \pm}$and $H_{h o r}^{ \pm}$denote, respectively, the vertical and horizontal components of the energy when $q_{z} \rightarrow \pm \infty$, i.e.:

$$
\begin{aligned}
& H_{\text {ver }}^{ \pm}=\lim _{t \rightarrow \pm \infty} \frac{1}{2 m} p_{z}{ }^{2}(t) \\
& H_{\text {hor }}^{ \pm}=\lim _{t \rightarrow \pm \infty} \frac{1}{2 m} p_{r}{ }^{2}(t)+\frac{A^{2}}{r^{2}(t)}+B^{2} r^{2}(t)+E_{c} .
\end{aligned}
$$

Clearly, the limits defined above exist. Furthermore, let us define the asymptotic changes in the vertical and horizontal energies, $\Delta H_{v e r}$ and $\Delta H_{h o r}$, by

$$
\begin{aligned}
\Delta H_{\text {ver }} & =H_{v e r}^{+}-H_{\text {ver }}^{-} \\
\Delta H_{\text {hor }} & =H_{\text {hor }}^{+}-H_{\text {hor }}^{-} .
\end{aligned}
$$

Theorem 5.8. If $\Omega_{1}=\Omega_{2}=\Omega$ then, for every level set $\{H=E\}$ of (5.37) such that $E>$ $E_{c}+2 A B$, the scattering map $S$ is well-defined. Furthermore, defining $E^{*}$ as in proposition 5.\%:

i) for every $E \in\left(E_{c}+A B, E^{*}\right)$ we have $p_{z}{ }^{+} p_{z}{ }^{-}<0$ and $q_{z}{ }^{+}=q_{z}{ }^{-}$, corresponding to "bouncing-back" behaviour.

ii) for every $E>E^{*}$ there is a mixture of "pass-through" and "bounce-back" behaviour.

Let $i \in\{1,2\}$. The scattering map also has the following properties:

a) typically for "pass-through" orbits there is exchange between parallel and perpendicular kinetic energy.

b) typically for "pass-through" orbits, the magnetic moments of the two particles are not conserved but they are always constrained by the following relations:

$$
\begin{aligned}
\frac{\mathrm{d}}{\mathrm{d} t}\left|k_{1} \boldsymbol{\rho}_{1}(t)+k_{2} \boldsymbol{\rho}_{2}(t)\right|^{2} & =0 \\
\lim _{t \rightarrow+\infty} k_{1}\left|\boldsymbol{\rho}_{1}(t)\right|^{2}+k_{2}\left|\boldsymbol{\rho}_{2}(t)\right|^{2} & -\lim _{t \rightarrow-\infty} k_{1}\left|\boldsymbol{\rho}_{1}(t)\right|^{2}+k_{2}\left|\boldsymbol{\rho}_{2}(t)\right|^{2}=-\frac{2}{\Omega} \Delta H_{\text {ver }} .
\end{aligned}
$$

c) typically for "pass-through" orbits, the distance of the guiding centres to the fixed centre of rotation $-\mathbf{J P} / \mu$ is not conserved during an interaction but is always constrained by

$$
\lim _{t \rightarrow+\infty}\left|\boldsymbol{R}_{i}(t)+\frac{\mathbf{J} \boldsymbol{P}}{\mu}\right|^{2}-\lim _{t \rightarrow-\infty}\left|\boldsymbol{R}_{i}(t)+\frac{\mathbf{J} \boldsymbol{P}}{\mu}\right|^{2}=-\left(\frac{2 m}{k_{i}}\right)^{2} \Delta H_{v e r}, \quad i \in\{1,2\} .
$$

Proof. The first part of the theorem has already been proved in this subsection. The constraints in the second part follow from a simple analysis of the reconstruction map of theorem 3.1 and the reduced Hamiltonian (5.37). So the main job is to prove that $\Delta H_{v e r}$ is typically non-zero for "pass-through" orbits. To see this,

$$
\frac{\mathrm{d} H_{v e r}}{\mathrm{~d} t}=\frac{p_{z} \dot{p}_{z}}{m}=G \frac{p_{z} q_{z}}{m R^{3}}
$$


SO

$$
\Delta H_{v e r}=G \int \frac{q_{z} p_{z}}{m R^{3}} \mathrm{~d} t .
$$

For "pass-through" trajectories this can be computed to first order in $G$ along trajectories of the case $G=0$. These have $p_{z}=m v$, constant (and we assume non-zero), $q_{z}=v t$ (choosing the origin of time at $\left.q_{z}=0\right), r^{2}=C+D \cos \left(\Omega\left(t-t_{0}\right)\right)$ with $C^{2}-D^{2}=A^{2} / B^{2}$ and $H_{h o r}=2 B^{2} C$. Thus to first order in $G$,

$$
\Delta H_{v e r}=G \int \frac{v^{2} t}{\left(v^{2} t^{2}+C+D \cos \left(\Omega\left(t-t_{0}\right)\right)\right)^{3 / 2}} \mathrm{~d} t .
$$

If $D=0$ the integrand is odd and the result is zero, but to first order in $D / C$ the result is

$$
\Delta H_{v e r}=-\frac{3}{2} G D v^{2} \sin \Omega t_{0} \int \frac{t \sin \Omega t}{\left(v^{2} t^{2}+C\right)^{5 / 2}} \mathrm{~d} t
$$

which is non-zero for $\sin \Omega t_{0} \neq 0$. Now $\Delta H_{v e r}$ is analytic in the initial conditions and $G$ for "pass-through" orbits, so being non-constant with respect to $D$ for small $G$ its zeroes in $D$ are isolated. Thus it is typically non-zero (though very small if $v^{2} \ll C \Omega^{2}$ ).

Remark Presumably, transfer between perpendicular and parallel kinetic energy is also typical in the "bounce-back" case, but a different proof would be required.

Using the two relations from item c) in the previous theorem it is possible to derive explicit formulae for the (forward) asymptotic gyroareas $2 \pi \rho_{i}^{+}$in terms of the value of the conserved quantity $W$, the variation of the vertical energy $\Delta H_{v e r}$, the (backward) asymptotic gyroareas $2 \pi \rho_{i}^{-}$and the forward relative gyrophase of the two particles $\Theta^{+}=\lim _{t \rightarrow+\infty}\left(\theta_{1}(t)-\theta_{2}(t)\right)$. Putting together (5.1), (5.2) and (5.40), we obtain

$$
\begin{aligned}
k_{1}^{2} \rho_{1}^{+}+2 k_{1} k_{2}\left(\rho_{1}^{+} \rho_{2}^{+}\right)^{1 / 2} \cos \left(\Theta^{+}\right)+k_{2}^{2} \rho_{2}^{+} & =\frac{W}{2} \\
k_{1} \rho_{1}^{+}+k_{2} \rho_{2}^{+} & =k_{1} \rho_{1}^{-}+k_{2} \rho_{2}^{-}-\frac{\Delta H_{v e r}}{\Omega} .
\end{aligned}
$$

Solving the equalities above with respect to $\rho_{1}^{+}$and $\rho_{2}^{+}$, we get

$$
\rho_{1}^{+}=\rho_{1}^{-}-\frac{k_{2}}{k_{1}}\left(\rho_{2}^{+}-\rho_{2}^{-}\right)-\frac{\Delta H_{v e r}}{k_{1} \Omega},
$$

where $\rho_{2}^{+}$is one of the (positive) solutions of the quadratic equation

$$
\frac{k_{1}^{2}}{k_{2}^{2}} \rho_{1}^{+2}+\frac{k_{2}^{2}}{k_{1}^{2}} \rho_{2}^{+2}+2\left(1-2 \cos \left(\Theta^{+}\right)\right) \rho_{1}^{+} \rho_{2}^{+}-W\left(\frac{\rho_{1}^{+}}{k_{2}^{2}}+\frac{\rho_{2}^{+}}{k_{1}^{2}}\right)-\frac{W^{2}}{4 k_{1}^{2} k_{2}^{2}}=0
$$

replacing $\rho_{1}^{+}$by the right hand side of equation (5.41).

The solutions of equations (5.41) and (5.42) are the intersection points in the $\left(\rho_{1}^{+}, \rho_{2}^{+}\right)$plane of the conic given by (5.42) with the line defined by (5.41).

- If $\Theta^{+}=0$ the conic (5.42) is a parabola and there are two (positive) intersection points with the line (5.42) provided that $\left|k_{1}\right| \rho_{1}^{-}+\left|k_{2}\right| \rho_{2}^{-}>M^{-}$, where $M^{-}$is given by

$$
M^{-}=\frac{\Delta H_{v e r}}{|\Omega|}+\frac{W}{2\left(\left|k_{1}\right|+\left|k_{2}\right|\right)} .
$$


- If $0<\Theta^{+}<\pi / 2$ the conic (5.42) is an ellipse and there are two (positive) intersection points with the line (5.42) whenever the inequalities $M^{-}<\left|k_{1}\right| \rho_{1}^{-}+\left|k_{2}\right| \rho_{2}^{-}<M^{+}$are satisfied, where $M^{+}$and $M^{-}$are given by

$$
M^{ \pm}=\frac{\Delta H_{v e r}}{|\Omega|}+W \frac{\left|k_{1}\right|+\left|k_{2}\right| \pm\left(\left(k_{1}-k_{2}\right)^{2}-4 k_{1} k_{2} \cos \left(\Theta^{+}\right)\right)^{1 / 2}}{4 k_{1} k_{2}\left(1-\cos \left(\Theta^{+}\right)\right)} .
$$

There is exactly one intersection when one of the inequalities becomes an equality.

- If $\Theta^{+}=\pi / 2$ the conic (5.42) degenerates to a line with slope $-k_{1}{ }^{2} / k_{2}{ }^{2}$ and there is one (positive) intersection point with the line (5.42) provided that $k_{1} \neq k_{2}$ and $M^{-}<$ $\left|k_{1}\right| \rho_{1}^{-}+\left|k_{2}\right| \rho_{2}^{-}<M^{+}$, where $M^{+}$and $M^{-}$are given by

$$
M^{+}=\frac{\Delta H_{v e r}}{|\Omega|}+\frac{W}{2 \min \left\{\left|k_{1}\right|,\left|k_{2}\right|\right\}}, \quad M^{-}=\frac{\Delta H_{v e r}}{|\Omega|}+\frac{W}{2 \max \left\{\left|k_{1}\right|,\left|k_{2}\right|\right\}} .
$$

- If $\pi / 2<\Theta^{+}<\pi$ the conic (5.42) is an hyperbola and there are no (positive) intersection points with the line (5.42).

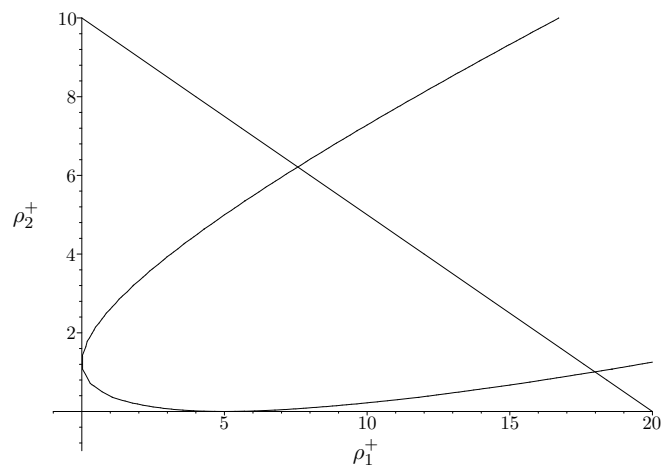

(a)

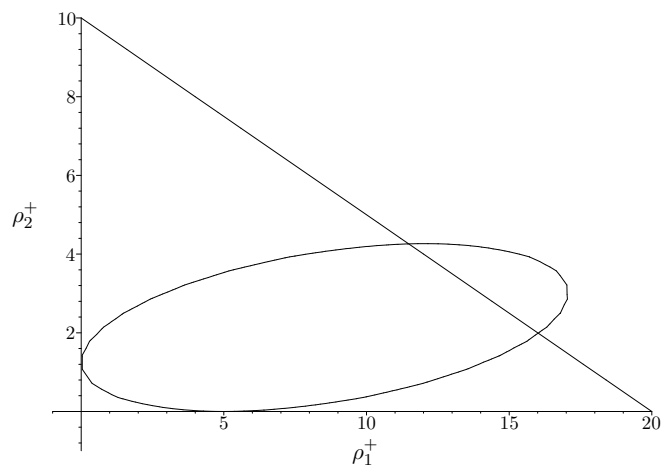

(b)

Figure 7: Intersections of conic sections of the form (5.42) with a line of the form (5.41). For both figures we fix the parameters $e_{1}=m_{1}=1, e_{2}=m_{2}=2, B=1, W=10, \Delta H_{v e r}=1$ and $\rho_{1}^{-}=\rho_{2}^{-}=7$. On the left figure $\Theta^{+}=0$ and on the right figure $\Theta^{+}=\pi / 4$.

5.3. Transfer of parallel energy in fixed frame. In the two previous sections we have done our analysis in a frame moving with the vertical centre of mass and studied the transfer of energy between the two particles. In this section we will see that in the original fixed frame there is a transfer of parallel kinetic energy between the particles in most cases of "bouncing-back" behaviour even if there is no change in the moving frame.

Assume the conditions of theorem 5.1 and recall that there exists $E^{\prime}>0$ such that for every level set $\left\{H^{\text {eff }}=E\right\}$ of $(5.21)$ with $0<E<E^{\prime}$ we have that $p_{z}{ }^{+}=-p_{z^{-}},\left|p_{z}{ }^{ \pm}\right|=(2 m E)^{1 / 2}$ and $q_{z}{ }^{+}=q_{z}{ }^{-}$, i.e. the particles "bounce-back" during an interaction.

Let $i \in\{1,2\}$ and let us denote by $p_{z_{i}}^{+}$(respectively $p_{z_{i}}^{-}$) the limit of $p_{z_{i}}(t)$ as $t \rightarrow+\infty$ (respectively $t \rightarrow-\infty$ ). By the reconstruction map in theorem 3.1 we obtain that

$$
p_{z_{1}}^{ \pm}=\frac{m_{1}}{M} P_{z}+p_{z}^{ \pm}, \quad p_{z_{2}}^{ \pm}=\frac{m_{2}}{M} P_{z}-p_{z}^{ \pm} .
$$


Thus, the change in the vertical kinetic energy of the first particle is given by

$$
\begin{aligned}
\Delta\left(K E_{1}\right) & =\frac{1}{2 m_{1}}\left(p_{z_{1}}^{+2}-{p_{z_{1}}^{-2}}^{2}\right) \\
& =\frac{2}{M} p_{z}^{+} P_{z} .
\end{aligned}
$$

Similarly, we obtain that the change in the vertical kinetic energy of the second particle is given by

$$
\begin{aligned}
\Delta\left(K E_{2}\right) & =\frac{1}{2 m_{2}}\left(p_{z_{2}}^{+2}-{p_{z_{2}}^{-}}^{2}\right) \\
& =-\frac{2}{M} p_{z}{ }^{+} P_{z} .
\end{aligned}
$$

Hence, if the vertical $z$-component of the centre of mass of the two particles moves with nonzero velocity $\dot{C}_{z}=P_{z} / M$ there is a transfer of vertical kinetic energy when the two particles "bounce-back".

5.4. Chaotic scattering: some numerical results. In this section we provide some numerical results that give strong evidence in favour of the existence of chaotic scattering for the problem of two charges of opposite sign moving in $\mathbb{R}^{3}$ under the action of a uniform magnetic field and a Coulomb interaction when the averaging assumptions of the previous subsection are not satisfied.

5.4.1. Procedure. We numerically integrate Hamilton's equations associated with the reduced Hamiltonian system given in theorem 3.1 under the following conditions: we fix the values of the parameters

$$
e_{1}=1, \quad m_{1}=1, \quad e_{2}=-3, \quad m_{2}=5, \quad B=1, \quad \epsilon_{0}=0.1,
$$

the initial conditions

$$
p_{r}(0)=0, \quad \phi(0)=0, \quad p_{\phi}(0)=1
$$

and the level sets of the conserved quantities

$$
p_{\theta}=1, \quad P_{z}=0 .
$$

Furthermore, if the energy $E>0$ and the initial conditions $r(0)=R$ and $q_{z}(0)=h>0$ are fixed, we obtain the value of the remaining initial condition $p_{z}(0)$ as a function of $E, R$ and $h$ :

$$
p_{z}(0)= \pm\left(\frac{3 E}{2}-\frac{49}{64 R^{2}}-\frac{25 R^{2}}{64}-\frac{5}{8}\left(R-\frac{7}{5 R}\right)+\frac{75}{4 \pi\left(R^{2}+h^{2}\right)^{1 / 2}}+\frac{21}{32}\right)^{1 / 2} .
$$

For a given $h>0$ choosing $p_{z}(0) \leq 0$ will make the particles move towards each other, interact and then move apart again.

We now fix the values of the energy $E$ and initial relative vertical position $q_{z}(0)=h$ and let $T_{E, h}(R)$ be the bigger instant of time needed for the particles starting with horizontal distance $r(0)=R$ (and corresponding $p_{z}(0)$ ) to reach a vertical distance of absolute value $h$ before escaping to $\infty$ when subject to a Coulomb interaction and let $T_{E, h}^{0}(R)$ be the time needed for the particles starting with the same initial conditions to reach a vertical distance $q_{z}\left(T_{E, h}^{0}(R)\right)=-h$ 
when moving freely in a uniform magnetic field. In the case of zero interaction the particles move with constant velocity in the vertical direction (equal to $(2 E / m)^{1 / 2}$ ) and hence we obtain

$$
T_{E, h}^{0}(R)=2 h \sqrt{\frac{m}{2 E}} .
$$

We define the time difference map $\tau_{E, h}: \mathbb{R}^{+} \rightarrow \mathbb{R}$ by

$$
\tau_{E, h}(R)=T_{E, h}(R)-T_{E, h}^{0}(R),
$$

and define the sign map $\sigma_{E, h}: \mathbb{R}^{+} \rightarrow\{+,-\}$ by

$$
\sigma_{E, h}(R)=\operatorname{sign}\left(q_{z}\left(T_{E, h}(R)\right)\right)
$$

where $\operatorname{sign}(x)$ denotes the sign of $x \in \mathbb{R}$. The sign map $\sigma_{E, h}(R)$ associates to each orbit the sign + if the particles "bounce-back" during the interaction and the sign - if the particles "pass-through".

Note that the definition given for the time difference map avoids the problems related with the unboundedness of the logarithmic term associated with the general time difference map (i.e. $h=\infty$ ) by making $h$ finite. However, we still have that $\tau_{E, h} \rightarrow \infty$ as $h \rightarrow \infty$.

5.4.2. Simulations. The plots for the maps $\sigma_{E, h}(R)$ and $\tau_{E, h}(R)$ that we show below were made for $h=500$ and values of energy $E=20, E=10, E=5$ and $E=1$.

Note that large energies lead to either a large vertical relative velocity or a large horizontal displacement between the two particles. For large vertical relative velocities the interaction time between the two particles is small while for large horizontal displacements the interaction strength is small. This is the main reason for the regularity of the dynamics for large energies and the lack of it for small energies. 


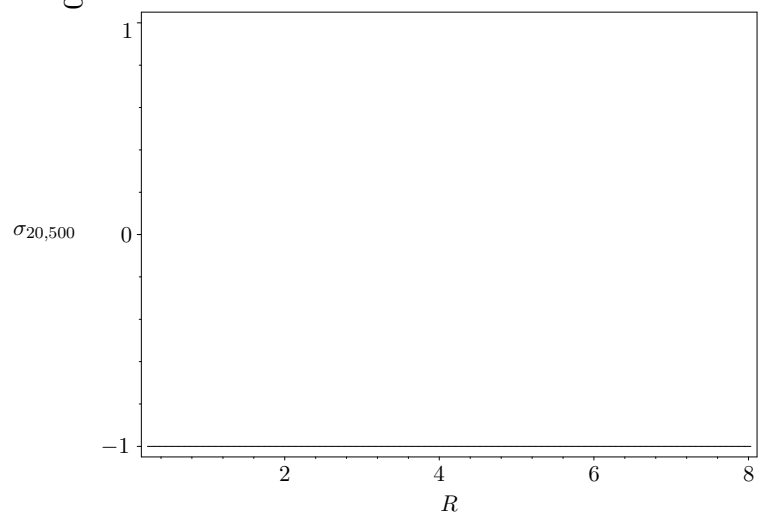

(a)

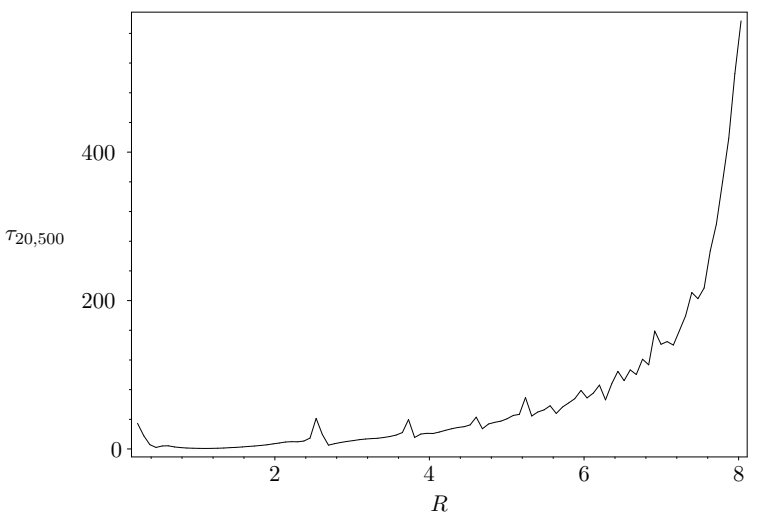

(b)

Figure 8: Plots of $\sigma_{E, h}(R)$ and $\tau_{E, h}(R)$ for $E=20$ and $h=500$. The motion is regular: the only type of dynamical behaviour observed is "pass-through" behaviour and the time difference map is reasonably smooth.

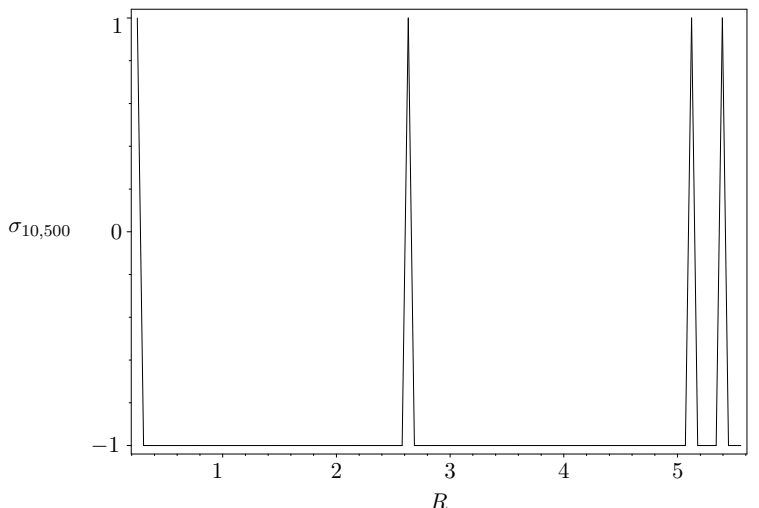

(a)

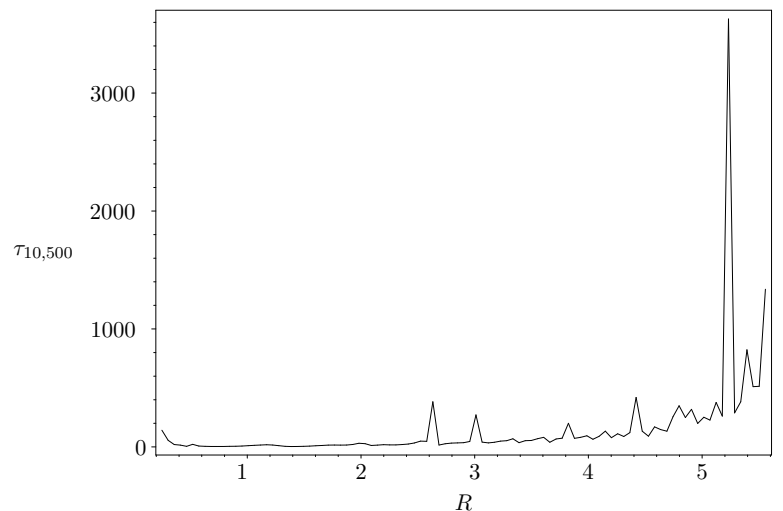

(b)

Figure 9: Plots of $\sigma_{E, h}(R)$ and $\tau_{E, h}(R)$ for $E=10$ and $h=500$. The motion is still mostly regular but there is already a small number of choices of initial horizontal distance that lead to a "bouncing-back" behaviour. The time difference map is still very regular. 


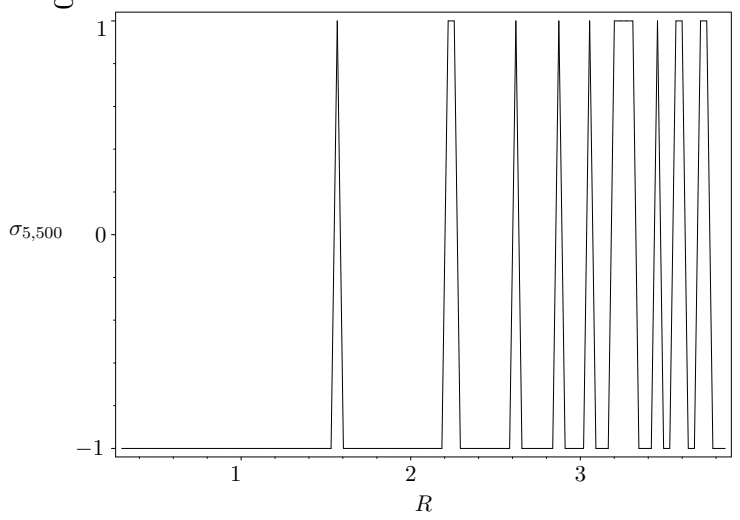

(a)

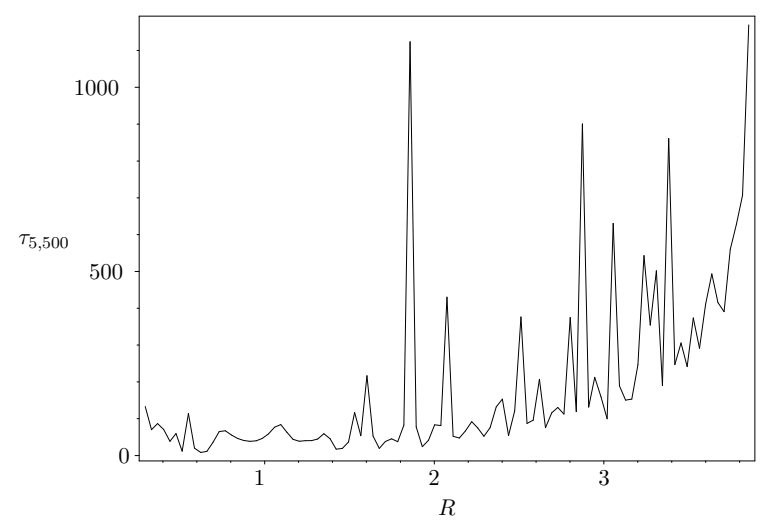

(b)

Figure 10: Plots of $\sigma_{E, h}(R)$ and $\tau_{E, h}(R)$ for $E=5$ and $h=500$. Although the dominant dynamical behaviour is "pass-through" there is already a large amount of "bouncing-back" behaviour. The time difference map loses much of its regularity.

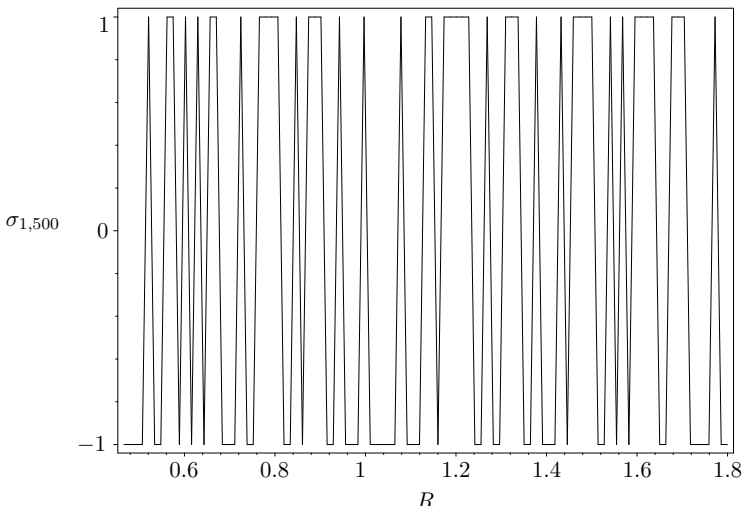

(a)

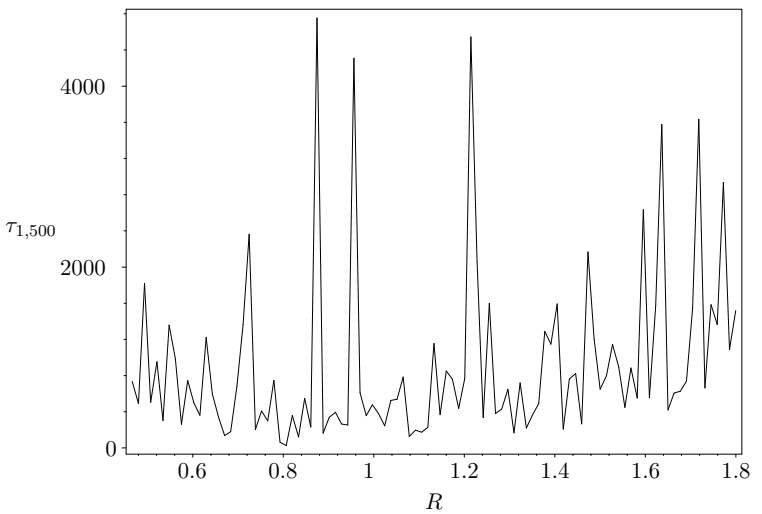

(b)

Figure 11: Plots of $\sigma_{E, h}(R)$ and $\tau_{E, h}(R)$ for $E=1$ and $h=500$. Chaotic scattering: both dynamical behaviours are observable and small changes in the initial horizontal distance might lead to each one of the observed behaviours. The time difference map is very irregular. 


\section{Conclusions}

We have proved that the Hamiltonian system (2.1) can always be reduced to one with three degrees of freedom. Moreover, we have proven that it can be reduced to one with two degrees of freedom for the special case of same sign charges when the particles have equal gyrofrequencies (equal ratio of charge to mass) and on some special submanifolds. Furthermore, we explicitly computed the reduced Hamiltonian systems and corresponding reconstruction maps, enabling us to lift the dynamics from the reduced spaces and hence obtain a description for the dynamics on the initial phase space.

In an invariant subspace where the reduced dynamics are just those associated with the interaction of two particles moving in a plane under the action of a uniform magnetic field (orthogonal to the plane of motion), we obtain that the system always reduces by a further degree of freedom, which leads to an integrable system in the case of two particles with equal gyrofrequency (and some special submanifolds). Specialising to the case of Coulomb interaction, as a consequence of the results in [19] we obtain that in the case of particles with opposite sign and non-zero gyrofrequencies sum, the system contains a suspension of a non-trivial subshift of finite type on all level sets of sufficiently high energy and hence it is non-integrable.

We have studied the scattering map associated with this problem in the regime where the two particles trajectories are widely separated. For rationally independent gyrofrequencies or high order rationally dependent gyrofrequencies, we have obtained that the magnetic moments are conserved to high accuracy, there is no change in parallel kinetic energy in the frame moving with the parallel centre of mass, and that the guiding centres either rotate about a fixed field line during an interaction in the case of two charges whose sum is not zero or translate in a direction determined by the linear momentum if the two charges sum is zero. We give explicit formulas for the rotations and translations in this regime. In the cases where the two particles have gyrofrequencies of equal absolute values we have proved that typically there is typically transfer of perpendicular kinetic energy between the particles, and in the case of equal gyrofrequencies there is also typically transfer between perpendicular and parallel kinetic energy. In a fixed frame, parallel kinetic energy is transferred between the particles in the case of "bounce-back", unless the vertical centre of mass speed is zero. This extends the results in $[2,7,8]$ from the case of infinite to finite magnetic field. To compute energy transport in a temperature or density gradient, however, one would also need to take into account the effects of the interaction-induced horizontal rotation (or translation) of the guiding centres that we have found, and the transfer of horizontal kinetic energy in the resonant cases, which move energy and particles horizontally in all cases of scattering.

We have also made a numerical study of the scattering map without using the assumption that the particles' trajectories are widely separated. We observed regular behaviour for large energies, but in the case of opposite signs of charge we found evidence for chaotic scattering for small positive energies.

One application of our results might be to reassess the derivations of kinetic equations for the velocity distribution functions of charged particles in a magnetic field, describing the effects of two-body scattering. The standard derivations (e.g. [5, 12]) appear to ignore the possibility of 
resonant interaction such as we have found for interaction of particles with equal gyrofrequencies. Even if the resonance effects might be significant only for a small fraction of interactions (those with low order rational ratio of gyrofrequencies and small relative parallel velocity), their net effect might turn out to be larger than the standard answers. By changing the Coulomb interaction to a Debye-shielded version, the Balescu-Lenard version of plasma kinetic theory could also be addressed. Any significant resulting changes to standard plasma kinetic theory could be valuable to understand the scattering of particles into the loss cone in the magnetosphere or that of particles into and out of banana orbits in tokamak fields. In particular, the result could shed light on the generation of toroidal current in tokamaks by such transitions and might contribute to the understanding of anomalous perpendicular electron heat transport.

\section{ACKnowledgments}

D. Pinheiro's research was supported by FCT - Fundação para a Ciência e Tecnologia grants with reference SFRH / BD / 9239 / 2002 and SFRH / BPD / 27151 / 2006 and CMUP - Centro de Matemática da Universidade do Porto. Parts of the work were done during visits of both of us to IMPA (Brazil) and IHES (France), whom we thank for their hospitality. We are grateful also for comments of Bob Dewar and Alan Kauffman on the results and relevant literature. We were saddened to learn of the death of John M. Greene on 22 October 2007, who had a profound influence on MacKay's career. With his mix of interest in plasma physics and Hamiltonian mechanics we think he would have liked the subject of this paper.

\section{REFERENCES}

[1] R. Abraham and J. Marsden. Foundations of Mechanics. Benjamin/Cummings (Reading, Massachusetts), 2nd edition, 1978.

[2] F. Anderegg, X. P. Huang, C. F. Driscoll, E. M. Hollmann, T. M. O'Neil, and D. H. E. Dubin. Test particle transport due to long range interactions. Physical Review Letters, 78:2128-2131, 1997.

[3] V. I. Arnold. Mathematical Methods of Classical Mechanics. Springer Verlag, 1989.

[4] J. E. Avron, I. W. Herbst, and B. Simon. Separation of Center of Mass in Homogeneous Magnetic Fields. Annals of Physics, 114:431-451, 1978.

[5] R. Balescu. Transport processes in plasma, vol.I. N.Holland, 1988.

[6] R. Cushman and L. Bates. Global Aspects of Classical Integrable Systems. Birkhäuser Verlag, 1997.

[7] C. F. Driscoll, F. Anderegg, D. H. E. Dubin, D. Z. Jin, J. M. Kriesel, E. M. Hollmann, and T. M. O'Neil. Shear reduction of collisional transport: experiments and theory. Physics of Plasmas, 9:1905-1914, 2002.

[8] D. H. E. Dubin. Collisional transport in non-neutral plasmas. Physics of Plasmas, 5:1688-1694, 1998.

[9] K. Efstathiou, R. H. Cushman, and Sadovskií. Hamiltonian Hopf bifurcation of the hydrogen atom in crossed fields. Physica D, 194:250-274, 2004.

[10] C. Grotta Raggazo, J. Koiller, and W. M. Oliva. On the motion of two-dimensional vortices with mass. J. Nonlinear Sci., 4:375-418, 1994.

[11] M. C. Gutzwiller. Chaos in Classical and Quantum Mechanics. Interdisciplinary applied mathematics. Springer Verlag, 1990.

[12] P. Helander and A. J. Sigmar. Collisional transport in magnetized plasmas. Cambridge Univ Press, 2002.

[13] R. G. Littlejohn. A guiding center Hamiltonian: a new approach. J Math Phys B, 20:2445-2458, 1979.

[14] P. Lochak and C. Meunier. Mutiphase Averaging for Classical Systems: With Applications to Adiabatic Theorems, volume 72 of Applied Mathematical Sciences. Springer-Verlag, 1988.

[15] R. S. MacKay. Flux over a saddle. Physics Letters A, 145(8,9):425-427, 1990.

[16] J. Marsden and T. Ratiu. Introduction to Mechanics and Symmetry. Springer Verlag, 1999. 
[17] E. Ott and T. Tél. Chaotic scattering: an introduction. Chaos, 3(4):417-426, 1993.

[18] D. Pinheiro. Interaction of two charges in a uniform magnetic field. $\mathrm{PhD}$ thesis, University of Warwick, 2006.

[19] D. Pinheiro and R. S. MacKay. Interaction of two charges in a uniform magnetic field: I. Planar problem. Nonlinearity, 19(8):1713-1745, 2006.

[20] M. Psimopoulos and D. Li. Cross field thermal transport in highly magnetized plamas. Proc. Royal Soc. Lond., 437:55-65, 1992.

[21] P. Schmelcher and L. S. Cederbaum. Regularity and chaos in the center of mass motion of the hydrogen atom in a magnetic field. Z. Phys. D, 24(4):311-323, 1992.

[22] P. Schmelcher and L. S. Cederbaum. Intermittent chaos in Hamiltonian systems: The three-dimensional hydrogen atom in magnetic fields. Physical Review A, 47(4):2634-2639, 1993.

[23] L. M. Tannenwald. Coulomb scattering in a very strong magnetic field. Physical Review, 113(6):1396-1405, 1959.

[24] G. Tanner, K. T. Hansen, and J. Main. The semiclassical resonance spectrum of hydrogen in a constant magnetic field. Nonlinearity, 9:1641-1670, 1996.

(D. Pinheiro) Centro de Matemática da Universidade do Porto, Departamentos de Matemática, Rua do Campo Alegre, 687, 4169-007 PORTO, Portugal

E-mail address: dpinheiro@fc.up.pt

(R. S. MacKay) Mathematics Institute, University of Warwick, Coventry CV4 7AL, UK

E-mail address: R.S.MacKay@warwick.ac.uk 